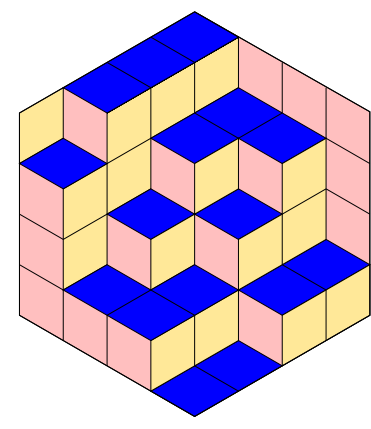

Alejandro H. Morales, Igor Pak \& Greta Panova

Hook formulas for skew shapes III. Multivariate and product formulas

Volume 2, issue 5 (2019), p. 815-861.

<http://alco.centre-mersenne.org/item/ALCO_2019__2_5_815_0>

(c) The journal and the authors, 2019.

Some rights reserved.

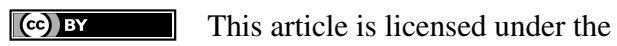

Creative Commons ATtribution 4.0 International LiCEnSE.

http://creativecommons.org/licenses/by/4.0/

Access to articles published by the journal Algebraic Combinatorics on the website http://alco.centre-mersenne.org/ implies agreement with the Terms of Use (http://alco.centre-mersenne.org/legal/).

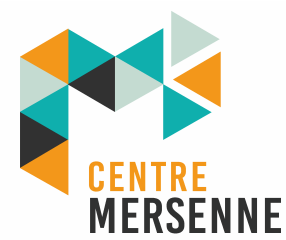

Algebraic Combinatorics is member of the Centre Mersenne for Open Scientific Publishing www.centre-mersenne.org 


\title{
Hook formulas for skew shapes III. Multivariate and product formulas
}

\author{
Alejandro H. Morales, Igor Pak \& Greta Panova
}

\begin{abstract}
We give new product formulas for the number of standard Young tableaux of certain skew shapes and for the principal evaluation of certain Schubert polynomials. These are proved by utilizing symmetries for evaluations of factorial Schur functions, extensively studied in the first two papers in the series $[54,52]$. We also apply our technology to obtain determinantal and product formulas for the partition function of certain weighted lozenge tilings, and give various probabilistic and asymptotic applications.
\end{abstract}

\section{INTRODUCTION}

1.1. ForEWORD. It is a truth universally acknowledged, that a combinatorial theory is often judged not by its intrinsic beauty but by the examples and applications. Fair or not, this attitude is historically grounded and generally accepted. While eternally challenging, this helps to keep the area lively, widely accessible, and growing in unexpected directions.

There are two notable types of examples and applications one can think of: artistic and scientific (cf. [19]). The former are unexpected results in the area which are both beautiful and mysterious. The fact of their discovery is the main application, even if they can be later shown by a more direct argument. The latter are results which represent a definitive progress in the area, unattainable by other means. To paraphrase Struik, this is "something to take home", rather than to simply admire (see [65]). While the line is often blurred, examples of both types are highly desirable, with the best examples being both artistic and scientific.

This paper is a third in a series and continues our study of the Naruse hooklength formula (NHLF), its generalizations and applications. In the first paper [54], we introduced two $q$-analogues of the NHLF and gave their (difficult) bijective proofs. In the second paper [52], we investigated the special case of ribbon hooks, which were used to obtain two new elementary proofs of NHLF in full generality, as well as various new mysterious summation and determinant formulas.

In this paper we present three new families of examples and applications of our tools:

Manuscript received 18th November 2017, revised 7th November 2018 and 24th June 2019, accepted 20th February 2019.

KEYWORDs. skew standard tableaux, product formulas, hook length, lozenge tilings, Schubert polynomials.

ACKNowledgements. The first author was partially supported by an AMS-Simons travel grant. The second and third authors were partially supported by the NSF. 
- new product formulas for the number of standard Young tableaux of certain skew shapes,

- new product formulas for the principal evaluation of certain Schubert polynomials,

- new determinantal formulas for weighted enumeration of lozenge tilings of a hexagon.

All three directions are so extensively studied from enumerative point of view, it is hard to imagine there is room for progress. In all three cases, we generalize a number of existing results within the same general framework of factorial Schur functions. With one notable exception (see \$9.4), we cannot imagine a direct combinatorial proof of the new product formulas circumventing our reasoning (cf. $\S 9.2$, however). As an immediate consequence of our results, we obtain exact asymptotic formulas which were unreachable until now (see sections 6 and 8). Below we illustrate our results one by one, leaving full statements and generalizations for later.

1.2. Number of SYT of SKew ShAPE. Standard Young tableaux are fundamental objects in enumerative and algebraic combinatorics and their enumeration is central to the area (see e.g. $[66,71]$ ). The number $f^{\lambda}=|\operatorname{SYT}(\lambda)|$ of standard Young tableaux of shape $\lambda$ and size $n$, is given by the classical hook-length formula:

$$
f^{\lambda}=n ! \prod_{u \in[\lambda]} \frac{1}{h(u)} .
$$

Famously, there is no general product formula for the number $f^{\lambda / \mu}=|\operatorname{SYT}(\lambda / \mu)|$ of standard Young tableaux of skew shape $\lambda / \mu{ }^{(1)}$ However, such formulas do exist for a few sporadic families of skew shapes and truncated shapes (see [1]).

In this paper we give a six-parameter family of skew shapes $\lambda / \mu=\Lambda(a, b, c, d, e, m)$ where $\mu=b^{a}$ with product formulas for the number of their SYT. The product formulas for these shapes of size $n$ include the MacMahon box formula and hooklengths of certain cells of $\lambda$ :

$$
f^{\lambda / \mu}=n ! \cdot \prod_{i=1}^{a} \prod_{j=1}^{b} \prod_{k=1}^{c} \frac{i+j+k-1}{i+j+k-2} \cdot \prod_{(i, j) \in \lambda / 0^{c} b^{a}} \frac{1}{h_{\lambda}(i, j)},
$$

(see Theorem 4.1 and Figure 5 for an illustration of the skew shape and the cells of $\lambda$ whose hook-lengths appear above). The three corollaries below showcase the most elegant special cases. We single out two especially interesting special cases: Corollary 1.1 due to its connection to the Selberg integral, and Corollary 1.2 due to its relation to shifted shapes and a potential for a bijective proof (see $\$ 9.4$ ). These two special cases were known before, but the corresponding proofs do not generalize to the setting of this paper.

The formulas below are written in terms of superfactorials $\Phi(n)$, double superfactorials $\Xi(n)$, super doublefactorials $\Psi(n)$, and shifted super doublefactorials $\Psi(n ; k)$ defined as follows:

$$
\begin{aligned}
\Phi(n) & :=1 ! \cdot 2 ! \cdots(n-1) !, & J(n) & :=(n-2) !(n-4) ! \cdots, \\
\Psi(n) & :=1 ! ! \cdot 3 ! ! \cdots(2 n-3) ! !, & \Psi(n ; k) & :=(k+1) ! ! \cdots(k+3) ! ! \cdots(k+2 n-3) ! !
\end{aligned}
$$

\footnotetext{
${ }^{(1)}$ In fact, even for small zigzag shapes $\pi=\delta_{k+2} / \delta_{k},<$ the number $f^{\pi}$ can have large prime divisors (cf. §5.3).
} 
Corollary 1.1 (Kim-Oh [25], see §9.3). For all $a, b, c, d, e \in \mathbb{N}$, let $\lambda / \mu$ be the skew shape in Figure $1(\mathrm{i})$. Then the number $f^{\lambda / \mu}=|\operatorname{SYT}(\lambda / \mu)|$ is equal to

$$
n ! \frac{\Phi(a) \Phi(b) \Phi(c) \Phi(d) \Phi(e) \Phi(a+b+c) \Phi(c+d+e) \Phi(a+b+c+d+e)}{\Phi(a+b) \Phi(d+e) \Phi(a+c+d) \Phi(b+c+e) \Phi(a+b+2 c+d+e)},
$$

where $n=|\lambda / \mu|=(a+c+e)(b+c+d)-a b-e d$.

Note that in [25, Cor. 4.7], the product formula is equivalent, but stated differently.

Corollary 1.2 (DeWitt [13], see §9.4). For all $a, b, c \in \mathbb{N}$, let $\lambda / \mu$ be the skew shape in Figure 1 (ii). Then the number $f^{\lambda / \mu}=|\operatorname{SYT}(\lambda / \mu)|$ is equal to

$$
n ! \frac{\Phi(a) \Phi(b) \Phi(c) \Phi(a+b+c) \cdot \Psi(c) \Psi(a+b+c)}{\Phi(a+b) \Phi(b+c) \Phi(a+c) \cdot \Psi(a+c) \Psi(b+c) \Psi(a+b+2 c)},
$$

where $n=|\lambda / \mu|=\left(\begin{array}{c}a+b+2 c \\ 2\end{array}\right)-a b$.

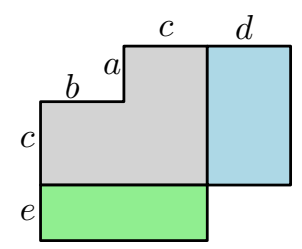

(i)

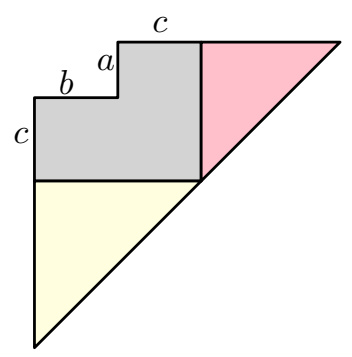

(ii)

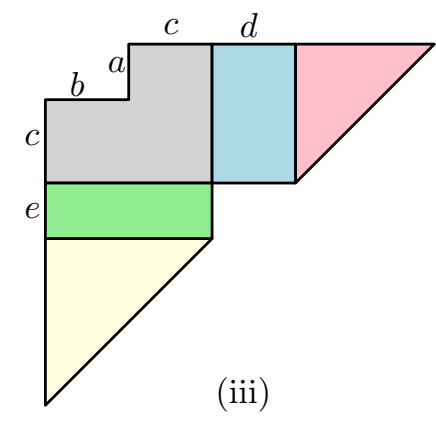

(iii)

FiguRE 1. Skew shapes $\Lambda(a, b, c, d, e, 0), \quad \Lambda(a, b, c, 0,0,1)$ and $\Lambda(a, b, c, d, e, 1)$ with product formulas for the number of SYT.

Corollary 1.3. For all $a, b, c, d, e \in \mathbb{N}$, let $\lambda / \mu$ be the skew shape in Figure 1 (iii). Then the number $f^{\lambda / \mu}=|\operatorname{SYT}(\lambda / \mu)|$ is equal to

$$
\begin{aligned}
& \frac{n ! \cdot \Phi(a) \Phi(b) \Phi(c) \Phi(a+b+c) \cdot \Psi(c ; d+e) \Psi(a+b+c ; d+e)}{\Phi(a+b) \Phi(b+c) \Phi(a+c) \cdot \Psi(a+c) \Psi(b+c) \Psi(a+b+2 c ; d+e)} \\
& \times \frac{\Xi(d) \beth(e) \beth(2 a+2 c) \beth(2 b+2 c)}{\beth(2 a+2 c+d) \beth(2 b+2 c+e)},
\end{aligned}
$$

Let us emphasize that the proofs of corollaries 1.1-1.3 are quite technical in nature. Here is a brief non-technical explanation. Fundamentally, the Naruse hook-length formula (NHLF) provides a new way to understand SYT of skew shape, coming from geometry rather than representation theory. What we show in this paper is that the proof of the NHLF has "hidden symmetries" which can be turned into product formulas (cf. §9.2). We refer to Section 4 for the complete proofs and common generalizations of these results, including $q$-analogues of the corollaries.

1.3. Product formulas for principal eValuations of Schubert polynomiALS. The Schubert polynomials $\mathfrak{S}_{w} \in \mathbb{Z}\left[x_{1}, \ldots, x_{n-1}\right], w \in S_{n}$, are generalizations of the Schur polynomials and play a key role in the geometry of flag varieties (see e.g. $[46,47])$. They can be expressed in terms of reduced words (factorizations) of the permutation $w$ via the Macdonald identity (8), and have been an object of intense 
study in the past several decades. In this paper we obtain several new product formulas for the principal evaluation $\mathfrak{S}_{w}(1, \ldots, 1)$ which has been extensively studied in recent years (see e.g. [7, 48, 69, 75, 79, 80]). Below we present two such formulas:

Corollary 1.4 (= Corollary 5.11). For the permutation $w(a, c):=1^{c} \times\left(2413 \otimes 1^{a}\right)$, where $c \geqslant a$, we have:

$$
\mathfrak{S}_{w(a, c)}(1,1, \ldots, 1)=\frac{\Phi(4 a+c) \Phi(c) \Phi(a)^{4} \Phi(3 a)^{2}}{\Phi(3 a+c) \Phi(a+c) \Phi(2 a)^{2} \Phi(4 a)} .
$$

Here $\sigma \times \omega$ and $\sigma \otimes \omega$ are the direct sum and the Kronecker product of permutations $\sigma$ and $\omega$ (see $\S 2.2$ ). We denote by by $1^{n}$ the identity permutation in $S_{n}$.

Corollary 1.5 (= Corollary 5.16). For the permutation $s(a):=351624 \otimes 1^{a}$, we have:

$$
\mathfrak{S}_{s(a)}(1,1, \ldots, 1)=\frac{\Phi(a)^{5} \Phi(3 a)^{2} \Phi(5 a)}{\Phi(2 a)^{4} \Phi(4 a)^{2}} .
$$

These results follow from two interrelated connections between principal evaluations of Schubert polynomials and the number of SYT of skew shapes. Below we give a brief outline, which is somewhat technical (see Section 2 for definitions and details).

The first connection is in the case of vexillary (2143-avoiding) permutations. The excited diagrams first appeared in a related context in work of Wachs [78] and Knutson-Miller-Yong [31], where they gave an explicit formula for the double Schubert polynomials of vexillary permutations in terms of excited diagrams (see §2.5) of a skew shape associated to the permutation. As a corollary, the principal evaluation gives the number of excited diagrams of the skew shape (Theorem 5.4). Certain families of vexillary permutations have skew shapes with product formulas for the number of excited diagrams (see above). Corollary 1.4 is one such example.

The second connection is in the case of 321-avoiding permutations. Combining the Macdonald identity and the results of Billey-Jockusch-Stanley [8], we show that the principal evaluation for 321-avoiding permutations is a multiple of $f^{\lambda / \mu}$ for a skew shape associated to the permutation (Theorem 5.13). In fact, every skew shape can be realized via a 321-avoiding permutation, see [8]. In particular, permutations corresponding to the skew shapes in $\S 1.2$ have product formulas for the principal evaluations. Corollary 1.5 follows along these lines from Corollary 1.1 with $a=b=$ $c=d=e$.

1.4. Determinantal Formulas For lozenge tilings. Lozenge tilings have been studied extensively in statistical mechanics and integrable probability, as exactly solvable dimer models on the hexagonal grid. When the tilings are chosen uniformly at random on a given domain and the mesh size $\rightarrow 0$, they have exhibited remarkable limit behavior like limit shape, frozen boundary, Gaussian Unitary Ensemble eigenvalue distributions, Gaussian Free Field fluctuations, etc. Such tilings are studied via a variety of methods ranging from variational principles (see e.g. [12, 24, 25]), to asymptotics of Schur functions and determinantal processes (see e.g. [10, 18, 62]).

Lozenge tilings of hexagonal shapes correspond naturally to plane partitions, when the lozenges are interpreted as sides of cubes and the tiling is interpreted as a projection of a stack of boxes. For example, lozenge tilings of the hexagon

$$
\mathrm{H}(a, b, c):=\langle a \times b \times c \times a \times b \times c\rangle
$$

are in bijection with solid partitions which fit inside the $[a \times b \times c]$ box. Thus, they are counted by the MacMahon box formula, see $\S 2.3$ :

$$
|\operatorname{PP}(a, b, c)|=\frac{\Phi(a+b+c) \Phi(a) \Phi(b) \Phi(c)}{\Phi(a+b) \Phi(b+c) \Phi(a+c)} .
$$


This connection allows us to translate our earlier results into the language of weighted lozenge tilings with multivariate weights on horizontal lozenges (Theorem 7.2). As a result, we obtain a number of determinantal formulas for the weighted sums of such lozenge tilings (see Figure 10: Right for weights of lozenges). Note that a similar but different extension of (2) to weighted lozenge tilings was given by Borodin, Gorin and Rains in [10]; see $\$ 9.6$ for a curious common special case of both extensions.

We then obtain new probabilistic results for random locally-weighted lozenge tilings. Specifically, observe that every vertical boundary edge is connected to an edge on the opposite side of the hexagon by a path $\mathrm{p}$, which goes through lozenges with vertical edges (see Figure 11(a)). Our main application is Theorem 8.2, which gives a determinant formula for the probability of $\mathrm{p}$ in the weighted lozenge tiling.
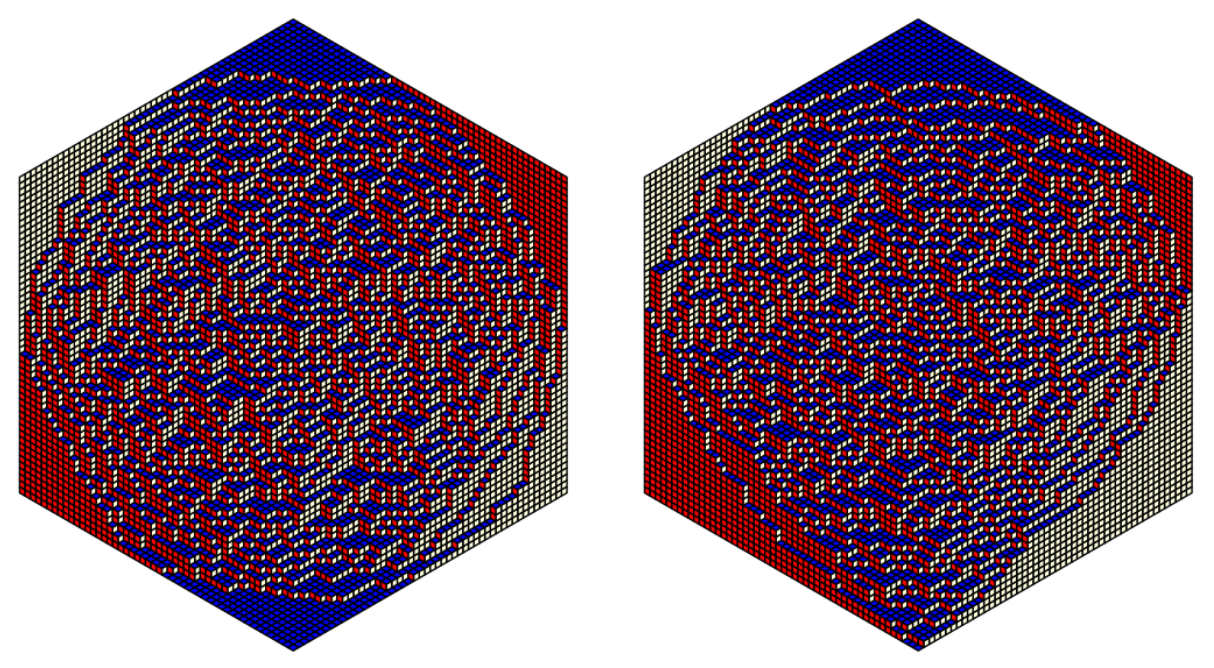

Figure 2. Random tilings of hexagon $\mathrm{H}(50,50,50)$ with uniform and hook weighted horizontal lozenges.

We illustrate the difference between the uniform and weighted lozenge tilings in Figure 2. Here both tilings of the hexagon $\mathrm{H}(50,50,50)$ are obtained by running the Metropolis algorithm for $2 \cdot 10^{9}$ steps. $^{(2)}$ In the latter case, the weight is defined to be a product over horizontal lozenges of a linear function in the coordinates (see Section 7 ). Note that the Arctic circle in the uniform case is replaced by a more involved limit shape as in the figure (see also Figure 17). In fact, the results in $\S 8.3$ explain why the latter limit shape is tilted upward, even if they are not strong enough to prove its existence (see $\S 9.8$ ).

1.5. Structure of the Paper. We begin with a lengthy Section 2 which summarizes the notation and gives a brief review of the earlier work. In the next Section 3 , we develop the technology of multivariate formulas including two key identities (Theorems 3.10 and 3.12). We use these identities to prove the product formulas for the number $f^{\lambda / \mu}$ of SYT of skew shape in Section 4, including generalization of corollaries 1.1-1.3. In Section 5 we use our technology to obtain product formulas for the principal evaluation of Schubert polynomials. These results are used in Section 6 to

${ }^{(2)}$ In the uniform case, a faster algorithm to generate such random tilings is given in [9] (see also [6]). 
obtain asymptotic formulas in a number of special cases. In Section 7, we obtain explicit determinantal formulas for the number of weighted lozenge tilings, which are then interpreted probabilistically and applied in two natural special cases in Section 8. We conclude with final remarks and open problems in Section 9.

\section{Notation And BACKGround}

2.1. Young Diagrams And Skew Shapes. Let $\lambda=\left(\lambda_{1}, \ldots, \lambda_{r}\right), \mu=\left(\mu_{1}, \ldots, \mu_{s}\right)$ denote integer partitions of length $\ell(\lambda)=r$ and $\ell(\mu)=s$. The size of the partition is denoted by $|\lambda|$ and $\lambda^{\prime}$ denotes the conjugate partition of $\lambda$. We use $[\lambda]$ to denote the Young diagram of the partition $\lambda$. The hook length $h_{\lambda}(i, j)=\lambda_{i}-i+\lambda_{j}^{\prime}-j+1$ of a square $u=(i, j) \in[\lambda]$ is the number of squares directly to the right or directly below $u$ in $[\lambda]$ including $u$.

A skew shape is denoted by $\lambda / \mu$ for partitions $\mu \subseteq \lambda$. The staircase shape is denoted by $\delta_{n}=(n-1, n-2, \ldots, 2,1)$. Finally, a skew shape $\lambda / \mu$ is called slim if $\lambda$ has $d$ parts and $\lambda_{d} \geqslant \mu_{1}+d-1$, see $[53, \S 11]$.

2.2. Permutations. We write permutations of $\{1,2, \ldots, n\}$ as $w=w_{1} w_{2} \ldots w_{n} \in$ $S_{n}$, where $w_{i}$ is the image of $i$. Given a positive integer $c$, let $1^{c} \times w$ denote the direct sum permutation

$$
1^{c} \times w:=12 \ldots c\left(c+w_{1}\right)\left(c+w_{2}\right) \ldots\left(c+w_{n}\right) .
$$

Similarly, let $w \otimes 1^{c}$ denote the Kronecker product permutation of size $c n$ whose permutation matrix equals the Kronecker product of the permutation matrix $P_{w}$ and the identity matrix $I_{c}$. See Figure $8(\mathrm{e})$ for an example.

To each permutation $w \in S_{n}$, we associate the subset of $[n] \times[n]$ given by

$$
D(w)=\left\{\left(i, w_{j}\right) \mid i<j, w_{i}>w_{j}\right\} .
$$

This set is called the (Rothe) diagram of $w$ and can be viewed as the complement in $[n] \times[n]$ of the hooks from the cells $\left(i, w_{i}\right)$ for $i=1,2, \ldots, n$. The size of this set is the length of $w$ and it uniquely determines $w$. Diagrams of permutations play in the theory of Schubert polynomials the role that partitions play in the theory of symmetric functions. The essential set of a permutation $w$ is given by

$$
E s s(w)=\{(i, j) \in D(w) \mid(i+1, j),(i, j+1),(i+1, j+1) \notin D(w)\} .
$$

See Figure 3 for an example of a diagram $D(w)$ and $\operatorname{Ess}(w)$.
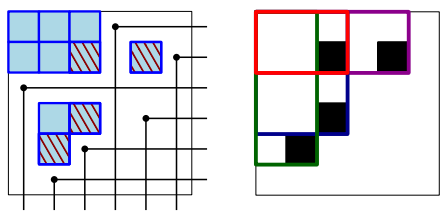

(a)
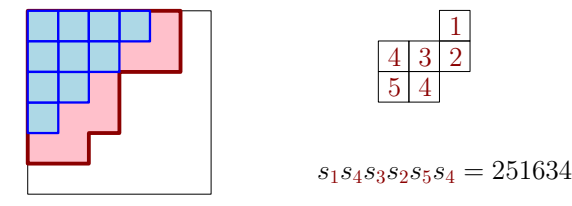

(b)

FiguRE 3. (a) The diagram of the vexillary permutation $w=461532$ (with cells in the essential set tiled in red). Up to permuting rows and columns it is the diagram of $\mu(w)=4321$; the supershape $\lambda(w)=$ 55332 defined by the essential set; the skew shape $\lambda(w) / \mu(w)$. (b) Example of correspondence between skew shapes and 321-avoiding permutations for $w=251634$.

The diagrams of two families of permutations have very appealing properties. These families are also described using the notion of pattern avoidance of permutations [30] 
and play an important role in Schubert calculus. We refer to [47, §2.1-2] for details and further examples.

A permutation is vexillary if $D(w)$ is, up to permuting rows and columns, the Young diagram of a partition denoted by $\mu=\mu(w)$. Equivalently, these are 2143avoiding permutations, i.e. there is no sequence $i<j<k<\ell$ such that $w_{j}<$ $w_{i}<w_{\ell}<w_{k}$. Given a vexillary permutation let $\lambda=\lambda(w)$ be the smallest partition containing the diagram $D(w)$. This partition is also the union over the $i \times j$ rectangles with NW-SE corners $(1,1),(i, j)$ for each $(i, j) \in \operatorname{Ess}(w)$. We call this partition the supershape of $w$ and note that $\mu(w) \subseteq \lambda(w)$ (see Figure 3(a)). Examples of vexillary permutations are dominant permutations (132-avoiding) and Grassmannian permutations (permutations with at most one descent).

A permutation is 321-avoiding if there is no sequence $i<j<k$ such that $w_{i}>$ $w_{j}>w_{k}$. The diagram $D(w)$ of such a permutation is, up to removing rows and columns of the board not present in the diagram and flipping columns, the Young diagram of a skew shape that we denote $\operatorname{skewsh}(w)$. Conversely, every skew shape $\lambda / \mu$ can be obtained from the diagram of a 321-avoiding permutation [8].

Theorem 2.1 (Billey-Jockusch-Stanley [8]). For every skew shape $\lambda / \mu$ with $(n-1)$ diagonals, there is a 321-avoiding permutation $w \in S_{n}$, such that $\operatorname{skewsh}(w)=\lambda / \mu$.

The construction from [8] to prove this theorem is as follows: Label the diagonals of $\lambda / \mu$ from right to left $d_{1}, d_{2}, \ldots$ and label the cells of $\lambda / \mu$ by the index of the their diagonal. Let $w$ be the permutation whose reduced word is obtained by reading the labeled cells of the skew shape from left to right top to bottom (see Figure 3(b)); we denote this reduced word by $\operatorname{rw}(\lambda / \mu)$. Note that $\operatorname{rw}(\lambda / \mu)$ is the lexicographically minimal among the reduced words of $w$.

2.3. Plane partitions. Let $\operatorname{PP}(a, b, c)$ and $\operatorname{RPP}(a, b, c)$ denote the sets of ordinary and reverse plane partitions $\pi$, respectively, that fit into an $[a \times b \times c]$ box with nonnegative entries, and let $|\pi|$ denote the sum of entries of the plane partition. Recall the MacMahon box formula (2) for the number of such (reverse) plane partitions, which can also be written as follows:

$$
|\operatorname{PP}(a, b, c)|=|\operatorname{RPP}(a, b, c)|=\prod_{i=1}^{a} \prod_{j=1}^{b} \prod_{k=1}^{c} \frac{i+j+k-1}{i+j+k-2},
$$

and its $q$-analogue:

$$
\sum_{\pi \in \operatorname{RPP}(a, b, c)} q^{|\pi|}=\prod_{i=1}^{a} \prod_{j=1}^{b} \prod_{k=1}^{c} \frac{1-q^{i+j+k-1}}{1-q^{i+j+k-2}} .
$$

2.4. Factorial Schur functions. The factorial Schur function (e.g. see [49]) is defined as

$$
s_{\mu}^{(d)}(\mathbf{x} \mid \mathbf{a}):=\frac{\operatorname{det}\left[\left(x_{i}-a_{1}\right) \cdots\left(x_{i}-a_{\mu_{j}+d-j}\right)\right]_{i, j=1}^{d}}{\Delta\left(x_{1}, \ldots, x_{d}\right)},
$$

where $\mathbf{x}=x_{1}, \ldots, x_{d}$ are variables, $\mathbf{a}=a_{1}, a_{2}, \ldots$ are parameters, and

$$
\Delta\left(x_{1}, \ldots, x_{d}\right)=\Delta(\mathbf{x}):=\prod_{1 \leqslant i<j \leqslant d}\left(x_{i}-x_{j}\right)
$$

is the Vandermonde determinant. By convention $\mu_{j}=0$ for $j>\ell(\mu)$. This function has an explicit expression in terms of semi-standard tableaux of shape $\mu$ :

$$
s_{\mu}^{(d)}(\mathbf{x} \mid \mathbf{a})=\sum_{T} \prod_{u \in \mu}\left(x_{T(u)}-a_{T(u)+c(u)}\right),
$$


where the sum is over semistandard Young tableaux $T$ of shape $\mu$ with entries in $\{1, \ldots, d\}$ and $c(u)=j-i$ denotes the content of the cell $u=(i, j)$. Moreover, $s_{\mu}^{(d)}(\mathbf{x} \mid \mathbf{a})$ is symmetric in $x_{1}, \ldots, x_{d}$.

2.5. Schubert Polynomials. Schubert polynomials were introduced by Lascoux and Schützen-berger [45] to study Schubert varieties. We denote by $\mathfrak{S}_{w}(\mathbf{x} ; \mathbf{y})$ the double Schubert polynomial of $w$ and by $\mathfrak{S}_{w}(\mathbf{x})=\mathfrak{S}_{w}(\mathbf{x} ; \mathbf{0})$ the single Schubert polynomial. See $[47, \S 2.3]$ and $[46, \S \mathrm{IV}, \mathrm{VI}]$ for definitions and properties.

The principal evaluation of a single Schubert polynomials at $x_{i}=1$, counting the number of monomials, is given by the following Macdonald identity [46, Eq. 6.11] (see also [47, Thm. 2.5.1] and [7] for a bijective proof):

$$
\Upsilon_{w}:=\mathfrak{S}_{w}(1,1, \ldots, 1)=\frac{1}{\ell !} \sum_{\left(r_{1}, \ldots, r_{\ell}\right) \in R(w)} r_{1} r_{2} \cdots r_{\ell} .
$$

Here $R(w)$ denotes the set of reduced words of $w \in S_{n}$ : tuples $\left(r_{1}, r_{2}, \ldots, r_{\ell}\right)$ such that $s_{r_{1}} s_{r_{2}} \cdots s_{r_{\ell}}$ is a reduced decomposition of $w$ into simple transpositions $s_{i}=(i, i+1)$.

2.6. ExCited Diagrams. Let $\lambda / \mu$ be a skew partition and $D$ be a subset of the Young diagram of $\lambda$. A cell $u=(i, j) \in D$ is called active if $(i+1, j),(i, j+1)$ and $(i+1, j+1)$ are all in $[\lambda] \backslash D$. Let $u$ be an active cell of $D$, define $\alpha_{u}(D)$ to be the set obtained by replacing $(i, j) \in D$ by $(i+1, j+1)$. We call this procedure an excited move. An excited diagram of $\lambda / \mu$ is a subdiagram of $\lambda$ obtained from the Young diagram of $\mu$ after a sequence of excited moves on active cells. Let $\mathcal{E}(\lambda / \mu)$ be the set of excited diagrams of $\lambda / \mu$.

EXAMPLE 2.2. The skew shape $\lambda / \mu=332 / 21$ has five excited diagrams:
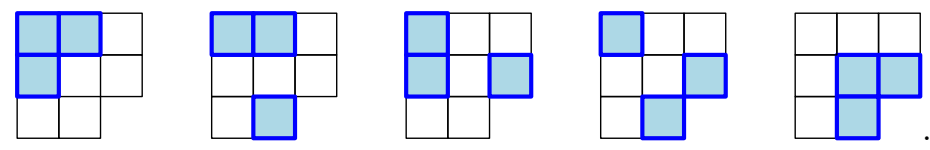

2.7. Flagged tableaux. Excited diagrams of $\lambda / \mu$ are equivalent to certain flagged tableaux of shape $\mu$ (see $[54, \S 3]$ and $[37, \S 6]$ ): SSYT of shape $\mu$ with bounds on the entries of each row. The number of excited diagrams is given by a determinant, a polynomial in the parts of $\lambda$ and $\mu$ as follows. Consider the diagonal that passes through cell $\left(i, \mu_{i}\right)$, i.e. the last cell of row $i$ in $\mu$. Let this diagonal intersect the boundary of $\lambda$ at a row denoted by $\mathrm{f}_{i}^{(\lambda / \mu)}$. Given an excited diagram $D$ in $\mathcal{E}(\lambda / \mu)$, each cell $(x, y)$ in $[\mu]$ corresponds to a cell $(i, j)$ in $D$, let $\varphi(D):=T$ be the tableau of shape $\mu$ with $T_{x, y}=i$.

Proposition 2.3 ([54]). The map $\varphi$ is a bijection between excited diagrams of $\lambda / \mu$ and SSYT of shape $\mu$ with entries in row $i$ at most $\mathrm{f}_{i}^{(\lambda / \mu)}$. Moreover,

$$
|\mathcal{E}(\lambda / \mu)|=\operatorname{det}\left[\left(\begin{array}{c}
\mathbf{f}_{i}^{(\lambda / \mu)}+\mu_{i}-i+j-1 \\
\mathrm{f}_{i}^{(\lambda / \mu)}-1
\end{array}\right)\right]_{i, j=1}^{\ell(\mu)} .
$$

Note that in the setting of this proposition, bounding all the entries of the SSYT is equivalent to bounding only the entries in the corners of this SSYT.

When the last part of $\lambda$ is long enough relative to the parts of $\mu$, the number of excited diagrams is given by a product. Recall the notion of slim shapes $\lambda / \mu$ defined in Section 2.1. 
Corollary 2.4. Let $\lambda / \mu$ be a slim skew shape, $d=\ell(\lambda)$. Then

$$
|\mathcal{E}(\lambda / \mu)|=s_{\mu}\left(1^{d}\right)=\prod_{(i, j) \in[\mu]} \frac{d+j-i}{\mu_{i}+\mu_{j}^{\prime}-i-j+1} .
$$

Proof. In this case, by Proposition 2.3, the excited diagrams of $\lambda / \mu$ are in bijection with SSYT of shape $\mu$ with entries at most $d$. The number of such SSYT is given by the hook-content formula for $s_{\mu}\left(1^{d}\right)$, see e.g. [72, Cor. 7.21.4].

Next we give a family of skew shapes that come up in the paper with product formulas for the number of excited diagrams.

EXAMPLE 2.5 (thick reverse hook). For the shape $\lambda / \mu=(b+c)^{a+c} / b^{a}$, the excited diagrams correspond to SSYT of shape $b^{a}$ with entries at most $a+c$. By subtracting $i$ from the elements in row $i$ these SSYT are equivalent to RPP that fit into an $[a \times b \times c]$ box. Thus, $|\mathcal{E}(\lambda / \mu)|=|\operatorname{RPP}(a, b, c)|$ is given by the MacMahon box formula (2).

2.8. Non-intersecting Paths. Excited diagrams of $\lambda / \mu$ are also in bijection with families of non-intersecting grid paths $\gamma_{1}, \ldots, \gamma_{k}$ with a fixed set of start and end points, which depend only on $\lambda / \mu$. A variant of this was proved by Kreiman [37, §5-6] (see also $[52, \S 3]$ ).

Formally, given a connected skew shape $\lambda / \mu$ let $\gamma_{1}^{*}$ be the path starting at the northwestern-most box, following the noertwest boundary and ending at the southeastern-most box of the connected component of this skew shape. Clearly, $(\lambda / \mu) \backslash \gamma_{1}^{*}$ will be a skew shape. We iterate the construction of the paths on each connected component of this new skew shape ordered bottom to top and obtain a family of non-intersecting paths $\gamma_{1}^{*}, \ldots, \gamma_{k}^{*}$ in $\lambda$ with support $\lambda / \mu$, where each path $\gamma_{i}^{*}$ starting at a box $\left(a_{i}, b_{i}\right)$ and ending at a box $\left(c_{i}, d_{i}\right)$. Let $\mathcal{N} \mathcal{I P}(\lambda / \mu)$ be the set of $k$-tuples $\Gamma:=\left(\gamma_{1}, \ldots, \gamma_{k}\right)$ of non-intersecting paths contained in $[\lambda]$ with $\gamma_{i}:\left(a_{i}, b_{i}\right) \rightarrow\left(c_{i}, d_{i}\right)$.

Proposition 2.6 (Kreiman [37], see also [52, §3.3]). Non-intersecting paths in $\mathcal{N I P}(\lambda / \mu)$ are uniquely determined by their support, i.e. set of squares. Moreover, the set of such supports is exactly the set of complements $[\lambda] \backslash D$ of excited diagrams $D \in \mathcal{E}(\lambda / \mu)$.

EXAMPLE 2.7. The complements of excited diagrams in $\mathcal{E}(444 / 21)$ correspond to tuples $\left(\gamma_{1}, \gamma_{2}\right)$ of nonintersecting paths in [444] with $\gamma_{1}=(3,1) \rightarrow(1,4)$ and $\gamma_{2}=$ $(3,3) \rightarrow(2,4)$ :

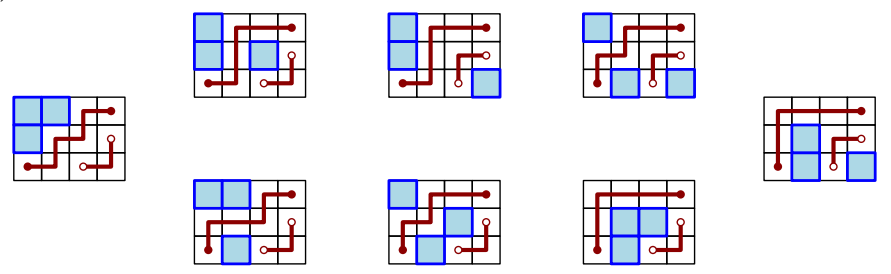

REMARK 2.8. The convention in [37, Lemma 5.3] and [52, §3.3] for the paths and starting/ending points is slightly different: the paths begin in the southern box of a column and end at the eastern box of a row instead. However, since the supports of excited diagram of a shape $\lambda / \mu$ only vary along the diagonals of $\mu$, then the portions of the paths outside this region will stay the same. Thus, it does not matter how the paths are drawn outside of this region.
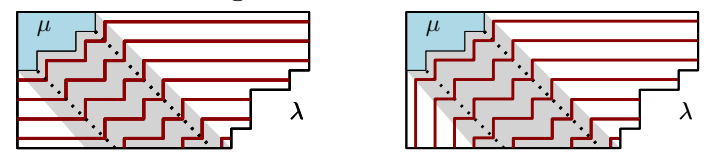
REMARK 2.9. The excited diagrams of a skew shape have a "path-particle duality" of sorts since they can be viewed as the cells or "particles" of the Young diagram of $\mu$ sliding down the cells of the Young diagram of $\lambda$ and also their complements are in correspondence with certain non-intersecting lattice paths. In the second part of the paper we give two other interpretations of excited diagrams as lozenge tilings and as terms in a known rule for Schubert polynomials of vexillary permutations (see $\S 7,5)$.

2.9. The Naruse hook-Length Formula. Recall the formula of Naruse for $f^{\lambda / \mu}$ as a sum of products of hook-lengths (see [54, 52]).

TheOREM 2.10 (NHLF; Naruse [57]). Let $\lambda, \mu$ be partitions, such that $\mu \subset \lambda$. We have:

$$
f^{\lambda / \mu}=n ! \sum_{D \in \mathcal{E}(\lambda / \mu)} \prod_{u \in[\lambda] \backslash D} \frac{1}{h(u)},
$$

where the sum is over all excited diagrams $D$ of $\lambda / \mu$.

For the $q$-analogues we use a $q$-analogue from [54] for skew semistandard Young tableaux.

Theorem 2.11 ([54]). We have:(3)

$$
s_{\lambda / \mu}\left(1, q, q^{2}, \ldots\right)=\sum_{D \in \mathcal{E}(\lambda / \mu)} \prod_{(i, j) \in[\lambda] \backslash D} \frac{q^{\lambda_{j}^{\prime}-i}}{1-q^{h(i, j)}} .
$$

These two results were the main object of our study in the two previous papers in the series $[54,52]$. It is also the key to most results in this paper. However, rather than apply it as "black box" we need to use the technology of multivariate sums in the proof of (NHLF).

2.10. Asympтотісs. We use the standard asymptotics notations $f \sim g, f=o(g)$, $f=O(g)$ and $f=\Omega(g)$, see e.g. [16, §A.2]. Recall Stirling's formula $\log n !=n \log n-$ $n+O(\log n)$. Here and everywhere below $\log$ denotes natural logarithm.

Below is a quick list of asymptotic formulas for other functions in the introduction:

$$
\begin{aligned}
& \log (2 n-1) ! !=n \log n+(\log 2-1) n+O(1), \\
& \log \Phi(n)=\frac{1}{2} n^{2} \log n-\frac{3}{4} n^{2}+O(n \log n), \\
& \log \Psi(n)=\frac{1}{2} n^{2} \log n+\left(\frac{\log 2}{2}-t s \frac{3}{4}\right) n^{2}+O(n \log n), \\
& \log I(n)=\frac{1}{4} n^{2} \log n-\frac{3}{8} n^{2}+O(n \log n),
\end{aligned}
$$

see [70, A001147], [70, A008793], [70, A057863], and [70, A113296]. We should also mention that the numbers $\Phi(n)$ are the integer values of the Barnes $G$-function, whose asymptotics has been extensively studied, see e.g. [3].

\section{Multivariate Path identity}

3.1. Multivariate sums of EXCited Diagrams. For the skew shape $\lambda / \mu \subseteq d \times$ $(n-d)$ we define $F_{\lambda / \mu}(\mathbf{x} \mid \mathbf{y})$ and $G_{\lambda / \mu}(\mathbf{x} \mid \mathbf{y})$ to be the multivariate sums of excited diagrams

\footnotetext{
${ }^{(3)}$ In [54], this is the first $q$-analogue of (NHLF). The second $q$-analogue is in terms of reverse plane partitions.
} 


$$
\begin{aligned}
G_{\lambda / \mu}(\mathbf{x} \mid \mathbf{y}) & :=\sum_{D \in \mathcal{E}(\lambda / \mu)} \prod_{(i, j) \in D}\left(x_{i}-y_{j}\right), \\
F_{\lambda / \mu}(\mathbf{x} \mid \mathbf{y}) & :=\sum_{D \in \mathcal{E}(\lambda / \mu)} \prod_{(i, j) \in[\lambda] \backslash D} \frac{1}{x_{i}-y_{j}} .
\end{aligned}
$$

By Proposition 2.6, the sum $F_{\lambda / \mu}(\mathbf{x} \mid \mathbf{y})$ can be written as a multivariate sum of non-intersecting paths.

COROLLARY 3.1. In the notation above, we have:

$$
F_{\lambda / \mu}(\mathbf{x} \mid \mathbf{y})=\sum_{\Gamma \in \mathcal{N} \mathcal{I P}(\lambda / \mu)} \prod_{(i, j) \in \Gamma} \frac{1}{x_{i}-y_{j}} .
$$

Note that by evaluating $F_{\lambda / \mu}(\mathbf{x} \mid \mathbf{y})$ at $x_{i}=\lambda_{i}-i+1$ and $y_{j}=-\lambda_{j}^{\prime}+j$ and multiplying by $|\lambda / \mu|$ ! we obtain the RHS of (NHLF).

$$
\left.F_{\lambda / \mu}(\mathbf{x} \mid \mathbf{y})\right|_{\substack{x_{i}=\lambda_{i}-i+1 \\ y_{j}=-\lambda_{j}^{\prime}+j}}=\frac{f^{\lambda / \mu}}{|\lambda / \mu| !} .
$$

Note that by evaluating $(-1)^{|\lambda / \mu|} F_{\lambda / \mu}(\mathbf{x} \mid \mathbf{y})$ at $x_{i}=q^{\lambda_{i}-i+1}$ and $y_{j}=q^{-\lambda_{j}^{\prime}+j}$ by $(q$-NHLF) we obtain

$$
\left.(-1)^{|\lambda / \mu|} F_{\lambda / \mu}(\mathbf{x} \mid \mathbf{y})\right|_{\substack{x_{i}=q^{\lambda_{i}-i+1} \\ y_{j}=q^{-\lambda_{j}^{\prime}+j}}}=q^{C(\lambda / \mu)} s_{\lambda / \mu}\left(1, q, q^{2}, \ldots\right),
$$

where

$$
C(\lambda / \mu)=\sum_{(i, j) \in \lambda / \mu}(j-i)
$$

The multivariate sum of excited diagrams can be written as an evaluation of a factorial Schur function.

Theorem 3.2 (see [21]). For a skew shape $\lambda / \mu$ inside the rectangle $d \times(n-d)$ we have:

$$
G_{\lambda / \mu}(\mathbf{x} \mid \mathbf{y})=s_{\mu}^{(d)}\left(y_{\lambda_{1}+d}, y_{\lambda_{2}+d-1}, \ldots, y_{\lambda_{d}+1} \mid y_{1}, \ldots, y_{n}\right) .
$$

EXAMPLE 3.3. Continuing with Example 2.5, take the thick reverse hook $\lambda / \mu=(b+$ $c)^{a+c} / b^{a}$. When we evaluate $G_{\lambda / \mu}(\mathbf{x} \mid \mathbf{y})$ at $x_{i}=q^{i}, y_{j}=0$, we obtain the $q$-analogue of the MacMahon box formula(4):

$$
G_{(b+c)^{a+c} / b^{a}}\left(q^{1}, q^{2}, \ldots \mid 0,0, \ldots\right)=q^{b\left(\begin{array}{c}
a+1 \\
2
\end{array}\right)} \prod_{i=1}^{a} \prod_{j=1}^{b} \prod_{k=1}^{c} \frac{1-q^{i+j+k-1}}{1-q^{i+j+k-2}} .
$$

Let $\mathbf{z}^{\langle\lambda\rangle}$ be the tuple of length $n$ of $x$ 's and $y$ 's by reading the horizontal and vertical steps of $\lambda$ from $(d, 1)$ to $(1, n-d)$ : i.e. $z_{\lambda_{i}+d-i+1}=x_{i}$ and $z_{d+j-\lambda_{j}^{\prime}}=y_{j}$. For example, for $d=4, n=9$ and $\lambda=(5533)$, we have $\mathbf{z}^{\langle\lambda\rangle}=\left(y_{1}, y_{2}, y_{3}, x_{4}, x_{3}, y_{4}, y_{5}, x_{2}, x_{1}\right)$ :

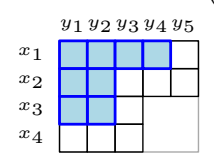

Combining results of Ikeda-Naruse [21], Knutson-Tao [32], Lakshmibai-RaghavanSankaran [42], one obtains the following formula for an evaluation of factorial Schur functions. 
LEMma 3.4 (Theorem 2 in [21]). For every skew shape $\lambda / \mu \subseteq d \times(n-d)$, we have:

$$
G_{\lambda / \mu}(\mathbf{x} \mid \mathbf{y})=s_{\mu}^{(d)}\left(\mathbf{x} \mid \mathbf{z}^{\langle\lambda\rangle}\right)
$$

Corollary 3.5. We have:

$$
F_{\lambda / \mu}(\mathbf{x} \mid \mathbf{y})=\frac{s_{\mu}^{(d)}\left(\mathbf{x} \mid \mathbf{z}^{\langle\lambda\rangle}\right)}{s_{\lambda}^{(d)}\left(\mathbf{x} \mid \mathbf{z}^{\langle\lambda\rangle}\right)}
$$

Proof. By definition the multivariate polynomial $G_{\lambda / \lambda}(\mathbf{x} \mid \mathbf{y})$ is the product $\prod_{(i, j) \in[\lambda]}\left(x_{i}-y_{j}\right)$ and thus we can write $F_{\lambda / \mu}(\mathbf{x} \mid \mathbf{y})$ as the following quotient

$$
F_{\lambda / \mu}(\mathbf{x} \mid \mathbf{y})=\frac{G_{\lambda / \mu}(\mathbf{x} \mid \mathbf{y})}{G_{\lambda / \lambda}(\mathbf{x} \mid \mathbf{y})}
$$

The result now follows by applying Lemma 3.4 to both the numerator and denominator on the RHS above.

3.2. Symmetries. The factorial Schur function $s_{\mu}^{(d)}(\mathbf{x} \mid \mathbf{y})$ is symmetric in $\mathbf{x}$. By Lemma 3.4, the multivariate sum $G_{\lambda / \mu}(\mathbf{x} \mid \mathbf{y})$ is an evaluation of a certain factorial Schur function, which in general is not symmetric in $\mathbf{x}$.

ExAmple 3.6. The shape $\lambda / \mu=332 / 21$ from Example 2.2 has five excited diagrams. One can check that the multivariate polynomial

$$
\begin{aligned}
& G_{333 / 21}\left(x_{1}, x_{2}, x_{3} \mid y_{1}, y_{2}, y_{3}\right)=\left(x_{1}-y_{1}\right)\left(x_{1}-y_{2}\right)\left(x_{2}-y_{1}\right) \\
& +\left(x_{1}-y_{1}\right)\left(x_{1}-y_{2}\right)\left(x_{3}-y_{2}\right)+\left(x_{1}-y_{1}\right)\left(x_{2}-y_{3}\right)\left(x_{2}-y_{1}\right) \\
& \quad+\left(x_{1}-y_{1}\right)\left(x_{2}-y_{3}\right)\left(x_{3}-y_{2}\right)+\left(x_{2}-y_{2}\right)\left(x_{2}-y_{3}\right)\left(x_{3}-y_{2}\right)
\end{aligned}
$$

is not symmetric in $\mathbf{x}=\left(x_{1}, x_{2}, x_{3}\right)$.

Now, below we present two cases when the sum $G_{\lambda / \mu}(\mathbf{x} \mid \mathbf{y})$ is in fact symmetric in $\mathbf{x}$. The first case is when $\mu$ is a rectangle contained in $\lambda$.

Proposition 3.7. Let $\mu=p^{k}$ be a rectangle, $p \geqslant k$, and let $\lambda$ be arbitrary partition containing $\mu$. Denote $\ell:=\max \left\{i: \lambda_{i}-i \geqslant p-k\right\}$. Then:

$$
G_{\lambda / p^{k}}(\mathbf{x} \mid \mathbf{y})=s_{p^{k}}^{(\ell)}\left(x_{1}, \ldots, x_{\ell} \mid y_{1}, \ldots, y_{p+\ell-k}\right) .
$$

In particular, the polynomial $G_{\lambda / p^{k}}(\mathbf{x} \mid \mathbf{y})$ is symmetric in $\left(x_{1}, \ldots, x_{\ell}\right)$.

Proof. First, observe that $\mathcal{E}\left(\lambda / p^{k}\right)=\mathcal{E}\left((p+\ell-k)^{\ell} / p^{k}\right)$ since the movement of the excited boxes is limited by the position of the corner box of $p^{k}$, which moves along the diagonal $j-i=p-k$ up to the boundary of $\lambda$, at position $(\ell, p+\ell-k)$. Thus, the excited diagrams of $\lambda / \mu$ coincide, as sets of boxes with the excited diagrams of $(p+\ell-k)^{\ell} / \mu$. Then:

$$
\begin{aligned}
G_{\lambda / p^{k}}(\mathbf{x} \mid \mathbf{y}) & =\sum_{D \in \mathcal{E}\left((p+\ell-k)^{\ell} / p^{k}\right)} \prod_{(i, j) \in D}\left(x_{i}-y_{j}\right)=G_{(p+\ell-k)^{\ell} / p^{k}}(\mathbf{x} \mid \mathbf{y}) \\
& =s_{p^{k}}^{(\ell)}\left(x_{1}, \ldots, x_{\ell} \mid \mathbf{z}^{\left\langle(p+\ell-k)^{\ell}\right\rangle}\right) .
\end{aligned}
$$

Note that $\mathbf{z}^{\left\langle(p+\ell-k)^{\ell}\right\rangle}=\left(y_{1}, \ldots, y_{p+\ell-k}, x_{\ell}, \ldots, x_{1}\right)$. Let us now invoke the original combinatorial formula for the factorial Schur functions, equation (7), with $a_{j}=y_{j}$ for $j \leqslant p+\ell-k$ and $a_{p+\ell-k+j}=x_{\ell+1-j}$ otherwise. Note also that when $T$ is an SSYT of shape $p^{k}$ and entries at most $\ell$, by the strictness of columns we have $T(i, j) \leqslant \ell-(k-i)$ for all entries in row $i$. We conclude:

$$
T(u)+c(u) \leqslant \ell-(k-i)+j-i=\ell-k+j \leqslant \ell-k+p .
$$


Therefore, $a_{T(u)+c(u)}=y_{T(u)+c(u)}$, where only the first $p+\ell-k$ parameters $a_{i}$ are involved in the formula. Then:

$$
\begin{aligned}
s_{\mu}^{(\ell)}\left(x_{\ell}, \ldots, x_{1} \mid a_{1}, \ldots, a_{p+\ell-k}, a_{p+\ell-k+1}, \ldots\right) & =s_{\mu}^{(\ell)}\left(x_{\ell}, \ldots, x_{1} \mid a_{1}, \ldots, a_{p+\ell-k}\right) \\
& =s_{\mu}^{(\ell)}\left(x_{1}, \ldots, x_{\ell} \mid y_{1}, \ldots, y_{p+\ell-k}\right),
\end{aligned}
$$

since now the parameters of the factorial Schur are independent of the variables $\mathbf{x}$ and the function is also symmetric in $\mathbf{x}$.

The second symmetry involves slim skew shapes (see Section 2.1). An example includes a skew shape $\lambda / \mu$, where $\lambda$ is the rectangle $(n-d)^{d}$ and $\mu_{1} \leqslant n-2 d+1$.

Proposition 3.8. Let $\lambda / \mu$ be a slim skew shape inside the rectangle $d \times(n-d)$. Then:

$$
G_{\lambda / \mu}(\mathbf{x} \mid \mathbf{y})=s_{\mu}^{(d)}\left(x_{1}, \ldots, x_{d} \mid y_{1}, \ldots, y_{\lambda_{d}}\right) .
$$

In particular, the polynomial $G_{\lambda / \mu}(\mathbf{x} \mid \mathbf{y})$ is symmetric in $\left(x_{1}, \ldots, x_{d}\right)$.

Proof. Note that $\mathbf{z}^{\langle\lambda\rangle}=\left(y_{1}, \ldots, y_{\lambda_{d}}, x_{d}, \ldots\right)$. Note also that for all $j=1, \ldots, d$,

$$
\mu_{j}+d-j \leqslant \lambda_{d}-d+1+d-j \leqslant \lambda_{d} \leqslant \lambda_{j}
$$

and so $z_{1}, \ldots, z_{\mu_{j}+d-j}=y_{1}, \ldots, y_{\mu_{j}+d-j}$. Next, we evaluate the factorial Schur function on the RHS of (13) via its determinantal formula (5). We obtain:

$$
\begin{aligned}
G_{\lambda / \mu}(\mathbf{x} \mid \mathbf{y}) & =s_{\mu}^{(d)}\left(x_{1}, \ldots, x_{d} \mid z_{1}, \ldots, z_{n}\right) \\
& =\frac{\operatorname{det}\left[\left(x_{d+1-i}-z_{1}\right) \cdots\left(x_{d+1-i}-z_{\mu_{j}+d-j}\right)\right]_{i, j=1}^{d}}{\Delta\left(x_{1}, \ldots, x_{d}\right)} \\
& =\frac{\operatorname{det}\left[\left(x_{i}-y_{1}\right) \cdots\left(x_{i}-y_{\mu_{j}+d-j}\right)\right]_{i, j=1}^{d}}{\Delta\left(x_{1}, \ldots, x_{d}\right)}=s_{\mu}^{(d)}\left(x_{1}, \ldots, x_{d} \mid y_{1}, \ldots, y_{\lambda_{d}}\right),
\end{aligned}
$$

where the last equality is by the same determinantal formula.

ExAmple 3.9 . For $\lambda / \mu=444 / 21$, the multivariate sum $G_{444 / 21}\left(x_{1}, x_{2}, x_{3}\right.$ $\left.y_{1}, y_{2}, y_{3}, y_{4}\right)$ of the eight excited diagrams in $\mathcal{E}(444 / 21)$ is symmetric in $x_{1}, x_{2}, x_{3}$.

3.3. Multivariate PATH IDEntities. We give two identities for the multivariate sums over non-intersecting paths as applications of each of Propositions 3.7 and 3.8.
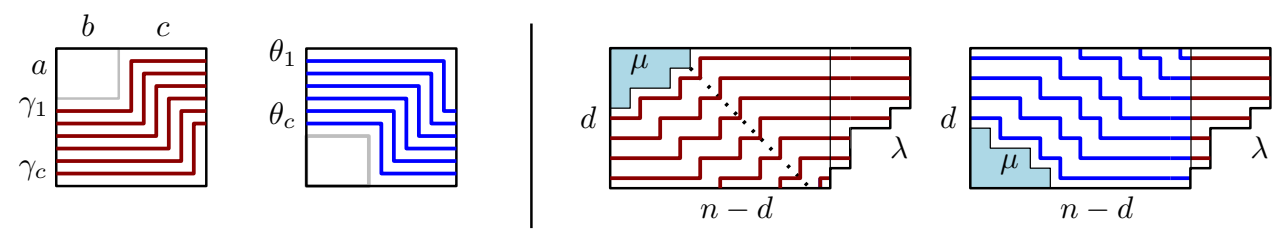

FiguRE 4. Left: paths and flipped paths in Theorem 3.10. Right: paths and flipped paths in Theorem 3.12.

THEOREM 3.10. We have the following identity for multivariate rational functions:

$$
\sum_{\substack{\Gamma=\left(\gamma_{1}, \ldots, \gamma_{c}\right) \\ \gamma_{p}:(a+p, 1) \rightarrow(p, b+c)}} \prod_{(i, j) \in \Gamma} \frac{1}{x_{i}-y_{j}}=\sum_{\substack{\Theta=\left(\theta_{1}, \ldots, \theta_{c}\right) \\ \theta_{p}:(p, 1) \rightarrow(a+p, b+c)}} \prod_{(i, j) \in \Theta} \frac{1}{x_{i}-y_{j}},
$$

where the sums are over non-intersecting lattice paths as above. Note that the LHS is equal to $F_{(b+c)^{a+c / b^{a}}}(\mathbf{x} \mid \mathbf{y})$ defined above. 
In the next section we use this identity to obtain product formulas for $f^{\lambda / \mu}$ for certain families of shapes $\lambda / \mu$. In the case $c=1$, we evaluate (15) at $x_{i}=i$ and $y_{j}=-j+1$ obtain the following corollary.

Corollary 3.11 ([54]). We have:

$$
\sum_{\gamma:(a, 1) \rightarrow(1, b)} \prod_{(i, j) \in \gamma} \frac{1}{i+j-1}=\sum_{\gamma:(1,1) \rightarrow(a, b)} \prod_{(i, j) \in \gamma} \frac{1}{i+j-1} .
$$

Equation (16) is a special case of (NHLF) for the skew shape $(b+1)^{a+1} / b^{a}[54$ $\S 3.1]$. This equation is also a special case of Racah formulas in $[10, \S 10]$ (see in $\S 9.6$ ).

Proof of Theorem 3.10. By Proposition 3.8 for the shape $(b+c)^{a+c} / b^{a}$, we have:

$$
G_{(b+c)^{a+c} / b^{a}}(\mathbf{x} \mid \mathbf{y})=s_{b^{a}}\left(x_{1}, \ldots, x_{a+c} \mid y_{1}, \ldots, y_{b+c}\right) .
$$

Divide the LHS by $\prod_{(i, j) \in(b+c)^{a+c}}\left(x_{i}-y_{j}\right)$ to obtain $F_{(b+c)^{a+c} / b^{a}}(\mathbf{x} \mid \mathbf{y})$, the multivariate sum over excited diagrams. By Corollary 3.1, this is also a multivariate sum over tuples of non-intersecting paths in $\mathcal{N} \mathcal{I P}\left((b+c)^{a+c} / b^{a}\right)$ :

$$
\sum_{\substack{\Gamma=\left(\gamma_{1}, \ldots, \gamma_{c}\right) \\ \gamma_{p}:(a+p, 1) \rightarrow(p, b+c)}} \prod_{(i, j) \in \Gamma} \frac{1}{x_{i}-y_{j}}=s_{b^{a}}\left(x_{1}, \ldots, x_{a+c} \mid y_{1}, \ldots, y_{b+c}\right) \prod_{(i, j) \in(b+c)^{a+c}} \frac{1}{x_{i}-y_{j}} .
$$

Finally, the symmetry in $x_{1}, \ldots, x_{a+c}$ of the RHS above implies that we can flip these variables and consequently the paths $\gamma_{p}^{\prime}$ to paths $\theta_{p}:(p, 1) \rightarrow(a+p, b+c)$ (see Figure 4), and obtain the needed expression.

For a partition $\mu$ inside the rectangle $d \times(n-d)$ of length $\ell$, let $\widehat{\mu}$ denote the tuple $\left(0^{d-\ell}, \mu_{\ell}, \mu_{\ell-1}, \ldots, \mu_{1}\right)$.

THEOREM 3.12. Let $\lambda / \mu \subset d \times(n-d)$ be a slim skew shape. Then:

$$
\sum_{\Gamma \in \mathcal{N} \mathcal{I} \mathcal{P}(\lambda / \mu)} \prod_{(i, j) \in \Gamma} \frac{1}{x_{i}-y_{j}}=\sum_{\Gamma \in \mathcal{N} \mathcal{I} \mathcal{P}(\lambda / \widehat{\mu})} \prod_{(i, j) \in \Gamma} \frac{1}{x_{i}-y_{j}}
$$

Proof. By Proposition 3.7 for the shape $\lambda / \mu$ we have that

$$
G_{(n-d)^{d} / \mu}(\mathbf{x} \mid \mathbf{y})=s_{\mu}^{(d)}\left(x_{1}, \ldots, x_{d} \mid y_{1}, \ldots, y_{\lambda_{d}}\right) .
$$

The rest of the proof follows mutatis mutandis that of Theorem 3.10 for the shape $\lambda / \mu$ instead of the shape $(b+c)^{a+c} / b^{a}$. See Figure 4 .

REMARK 3.13. In [55] we use this second symmetry identity to give new lower bounds on $f^{\lambda / \mu}$ for several other families of slim shapes $\lambda / \mu$.

3.4. VARIANT OF EXCITED DIAGRAMS FOR RECTANGLES AND SLIM SHAPES. Recall that for $\mu \subseteq d \times(n-d)$ of length $\ell$, we denote by $\widehat{\mu}$ the tuple $\left(\mathbf{0}^{d-\ell}, \mu_{\ell}, \mu_{\ell-1}, \ldots, \mu_{1}\right)$. We interpret the complements of the supports of the paths in $\mathcal{N} \mathcal{I P}\left((b+c)^{a+c} / 0^{c} b^{a}\right)$ and in $\mathcal{N} \mathcal{I P}(\lambda / \widehat{\mu})$, as variants of excited diagrams.

A $N E$-excited diagram of shape $\lambda / \mu$ is a subdiagram of $\lambda$ obtained from the Young diagram of $\widehat{b^{a}}=\left(0^{c} b^{a}\right)$ (and $\widehat{\mu}$ ) after a sequence of moves from $(i, j)$ to $(i-1, j+1)$ provided $(i, j)$ is in the subdiagram $D$ and all of $(i-1, j),(i-1, j+1),(i, j+1)$ are in $[\lambda] \backslash D$. We denote the set of such diagrams by $\mathcal{E}^{\nearrow}(\lambda / \mu)$. Analogous to Proposition 2.6, the complements of these diagrams correspond to tuples of paths in $\mathcal{N} \mathcal{I} \mathcal{P}\left((b+c)^{a+c} / 0^{c} b^{a}\right)$ (in $\left.\mathcal{N} \mathcal{I} \mathcal{P}(\lambda / \widehat{\mu})\right)$. Flipping horizontally the $\left[d \times \lambda_{d}\right]$ rectangle gives a bijection between excited diagrams and NE-excited diagrams of $\lambda / \mu$. Thus

$$
\left|\mathcal{E}^{\nearrow}(\lambda / \mu)\right|=|\mathcal{E}(\lambda / \mu)| \text {. }
$$


Moreover, equation (18) states that such a flip also preserves the multivariate series $F_{\lambda / \mu}(\mathbf{x} \mid \mathbf{y})$ and polynomial $G_{\lambda / \mu}(\mathbf{x} \mid \mathbf{y})$.

Corollary 3.14. We have:

$$
F_{(b+c)^{a+c / b^{a}}}(\mathbf{x} \mid \mathbf{y})=\sum_{D \in \mathcal{E}^{\nearrow}\left((b+c)^{a+c} / 0^{c} b^{a}\right)} \prod_{(i, j) \in[\lambda] \backslash D} \frac{1}{x_{i}-y_{j}},
$$

and

$$
G_{(b+c)^{a+c} / b^{a}}(\mathbf{x} \mid \mathbf{y})=\sum_{D \in \mathcal{E}^{\nearrow}\left((b+c)^{a+c} / 0^{c} b^{a}\right)} \prod_{(i, j) \in D}\left(x_{i}-y_{j}\right) .
$$

Proof. This follows from the discussion above, Corollary 3.1 and Theorem 3.10.

COROllary 3.15. For a slim skew shape $\lambda / \mu$, we have:

$$
F_{\lambda / \mu}(\mathbf{x} \mid \mathbf{y})=\sum_{D \in \mathcal{E}^{\nearrow}(\lambda / \mu)} \prod_{(i, j) \in[\lambda] \backslash D} \frac{1}{x_{i}-y_{j}} .
$$

Proof. This follows from the discussion above, Corollary 3.1 and Theorem 3.12.

\section{Skew Shapes With PRODUCT FORMUlas}

In this section we use Theorem 3.10 to obtain product formulas for a family of skew shapes.

4.1. SiX-Parameter Family OF SKew Shapes. For all $a, b, c, d, e, m \in \mathbb{N}$, let $\Lambda(a, b, c, d, e, m)$ denote the skew shape $\lambda / b^{a}$, where $\lambda$ is given by

$$
\lambda:=(b+c)^{a+c}+\left(\nu \cup \theta^{\prime}\right),
$$

and where $\nu=(d+(a+c-1) m, d+(a+c-2) m, \ldots, d), \theta=(e+(b+c-1) m, e+$ $(b+c-2) m, \ldots, e)$; see Figure 5 . This shape satisfies two key properties:

$$
\begin{aligned}
& \lambda_{a+c+1} \leqslant b+c, \\
& \lambda_{i}+\lambda_{j}^{\prime}=\lambda_{r}+\lambda_{s}^{\prime}, \quad \text { if } i+j=r+s \text { and }(i, j),(r, s) \in(b+c)^{a+c} .
\end{aligned}
$$

The second property implies that $\lambda_{i}-\lambda_{i+1}=\lambda_{j}^{\prime}-\lambda_{j+1}^{\prime}$ for all $i \leqslant a+c-1$ and $j \leqslant b+c-1$, and therefore $\lambda_{i}-\lambda_{i+1}$ is independent of $i$, i.e. the parts of $\lambda$ are given by an arithmetic progression. Also, the antidiagonals in $(b+c)^{a+c}$ inside $\lambda$ have the same hook-lengths.

Here are two extreme special cases:

$$
\Lambda(a, b, c, 0,0,1)=\delta_{a+b+2 c} / b^{a}, \quad \Lambda(a, b, c, d, e, 0)=(b+c+d)^{a+c}(b+c)^{e} / b^{a} .
$$

Note that these shapes are depicted in Figure 1(ii) and Figure 1(i), respect.

Next, we give a product formula for $f^{\pi}$ where $\pi=\Lambda(a, b, c, d, e, m)$ in terms of falling superfactorials

$$
\Psi^{(m)}(n):=\prod_{i=1}^{n-1} \prod_{j=1}^{i}(j m+j-1)_{m}, \quad \text { where } \quad(k)_{m}=k(k-1) \cdots(k-m+1) .
$$

Note that $\Psi^{(0)}(n)=1$ and $\Psi^{(1)}(n)=\Psi(n)$. 

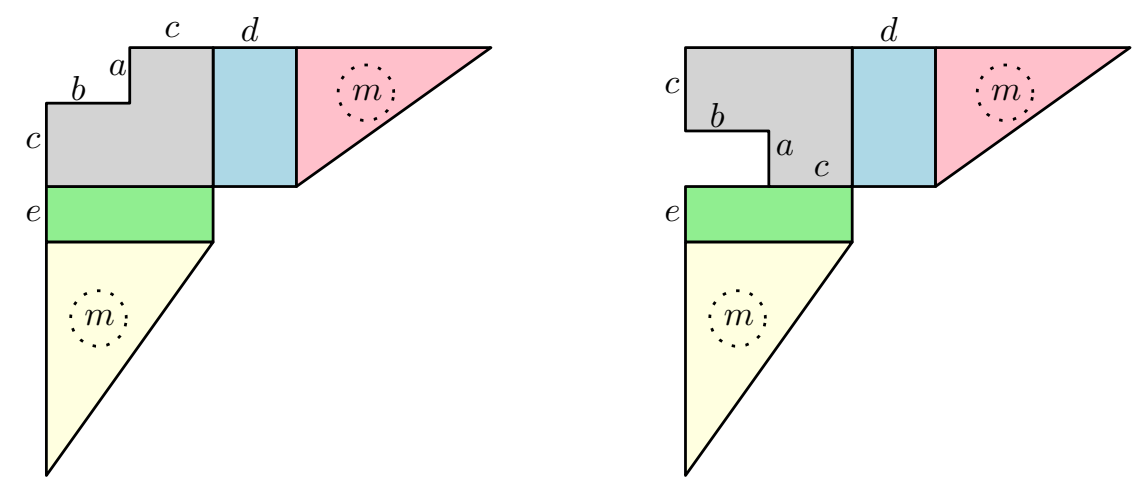

Figure 5. Left: Skew shape $\Lambda(a, b, c, d, e, m)$. Right: the cells whose hook-lengths appear in the product formula of Theorem 4.1.

TheOREM 4.1. Let $\pi=\Lambda(a, b, c, d, e, m)$ be as above. Then $f^{\pi}$ is given by the following product:

$$
\begin{aligned}
f^{\pi}=n ! & \cdot \prod_{i=1}^{a} \prod_{j=1}^{b} \prod_{k=1}^{c} \frac{i+j+k-1}{i+j+k-2} \cdot \prod_{(i, j) \in \lambda /\left(0^{c} b^{a}\right)} \frac{1}{h_{\lambda}(i, j)}, \\
(24)=n ! & \cdot \frac{\Phi(a+b+c) \Phi(a) \Phi(b) \Phi(c)}{\Phi(a+b) \Phi(b+c) \Phi(a+c) \Psi^{(m)}(a+c) \Psi^{(m)}(b+c)} \prod_{i=0}^{a+c-1} \frac{(i(m+1)) !}{(d+i(m+1)) !} \\
(25) \quad & \times \prod_{i=0}^{b+c-1} \frac{(i(m+1)) !}{(e+i(m+1)) !} \times \frac{\prod_{i=0}^{b-1} \prod_{j=0}^{a-1}(1+d+e+(c+i+j)(m+1))}{\prod_{i=0}^{b+c-1} \prod_{j=0}^{a+c-1}(1+d+e+(i+j)(m+1))} .
\end{aligned}
$$

Proof of Corollaries 1.1, 1.2 and 1.3. Use (25) for the shapes $\Lambda(a, b, c, e, d, 0)$, $\Lambda(a, b, c, 0,0,1)$, and $\Lambda(a, b, c, d, e, 1)$, respectively.

We also give a product formula for the generating function of SSYT of these shapes.

Theorem 4.2. Let $\pi=\Lambda(a, b, c, d, e, m)$ be as above. Then:

(26) $s_{\pi}\left(1, q, q^{2}, \ldots\right)=q^{N} \prod_{i=1}^{a} \prod_{j=1}^{b} \prod_{k=1}^{c} \frac{1-q^{(m+1)(i+j+k-1)}}{1-q^{(m+1)(i+j+k-2)}} \prod_{(i, j) \in \lambda /\left(0^{c} b^{a}\right)} \frac{1}{1-q^{h_{\lambda}(i, j)}}$,

where $N=\sum_{(i, j) \in \lambda / b^{a}}\left(\lambda_{j}^{\prime}-i\right)$.

As in the proof above, we obtain explicit formulas for the skew shapes $\Lambda(a, b, c, d, e, 0), \Lambda(a, b, c, 0,0,1)$, and $\Lambda(a, b, c, d, e, 1)$. By comparing (25) and (26), up to the power of $q$, these cases are obtained by " $q$-ifying" their counterpart formulas for $f^{\lambda / \mu}$. The formulas are written in terms of:

$$
\begin{array}{ll}
q \text {-factorials } & {[m] !:=(1-q)\left(1-q^{2}\right) \cdots\left(1-q^{m}\right)} \\
q \text {-double factorials } & {[2 n-1] ! !:=(1-q)\left(1-q^{3}\right) \cdots\left(1-q^{2 n-1}\right)} \\
q \text {-superfactorials } & \Phi_{q}(n):=[1] ! \cdot[2] ! \cdots[n-1] ! \\
q \text {-super doublefactorials } & \Psi_{q}(n):=[1] ! ! \cdot[3] ! ! \cdots[2 n-3] ! ! \\
q \text {-double superfactorial } & \Xi_{q}(n):=[n-2] ![n-4] ! \cdots \\
q \text {-shifted super doublefactorial } & \Psi_{q}(n ; k):=[k+1] ! ![k+3] ! ! \cdots[k+2 n-3] ! !
\end{array}
$$


Note that in the classical notation $[m] !=\prod \frac{1-q^{i}}{1-q}$ (e.g. from [74]), however here the factors of $(1-q)$ are omitted, otherwise the formulas below would have an additional factor of $(1-q)^{|\pi|}$.

Corollary 4.3. For the skew shape $\pi=\Lambda(a, b, c, d, e, 0)$, we have:

$$
\begin{aligned}
& s_{\pi}(1, q, \ldots)= \\
& \frac{q^{N} \Phi_{q}(a) \Phi_{q}(b) \Phi_{q}(c) \Phi_{q}(d) \Phi_{q}(e) \Phi_{q}(a+b+c) \Phi_{q}(c+d+e) \Phi_{q}(a+b+c+d+e)}{\Phi_{q}(a+b) \Phi_{q}(d+e) \Phi_{q}(a+c+d) \Phi_{q}(b+c+e) \Phi_{q}(a+b+2 c+d+e)},
\end{aligned}
$$

where $N=b\left(\begin{array}{c}c+e \\ 2\end{array}\right)+c\left(\begin{array}{c}a+c+e \\ 2\end{array}\right)+d\left(\begin{array}{c}a+c \\ 2\end{array}\right)$.

Note that this is a $q$-analogue of Corollary 4.3 by Kim and Oh (see also $§ 9.3$ ).

COROLlary 4.4 (Krattenthaler-Schlosser [36], see §9.4). For the skew shape $\pi=$ $\Lambda(a, b, c, 0,0,1)$, we have:

$$
\begin{aligned}
& s_{\pi}(1, q, \ldots)=q^{N} \frac{\Phi_{q}(a) \Phi_{q}(b) \Phi_{q}(c) \Phi_{q}(a+b+c) \cdot \Psi_{q}(c) \Psi_{q}(a+b+c)}{\Phi_{q}(a+b) \Phi_{q}(b+c) \Phi_{q}(a+c) \cdot \Psi_{q}(a+c) \Psi_{q}(b+c) \Psi_{q}(a+b+2 c)}, \\
& \text { where } N=\left(\begin{array}{c}
a+b+2 c \\
3
\end{array}\right)+b\left(\begin{array}{c}
a+1 \\
2
\end{array}\right)+a\left(\begin{array}{c}
b+1 \\
2
\end{array}\right)-a b(a+b+2 c) .
\end{aligned}
$$

Corollary 4.5. For the skew shape $\pi=\Lambda(a, b, c, d, e, 1)$, we have:

$$
\begin{aligned}
& s_{\pi}(1, q, \ldots)=q^{N} \frac{\Phi_{q}(a) \Phi_{q}(b) \Phi_{q}(c) \Phi_{q}(a+b+c)}{\Phi_{q}(a+b) \Phi_{q}(b+c) \Phi_{q}(a+c)} \\
& \times \frac{\Psi_{q}(c ; d+e) \Psi_{q}(a+b+c ; d+e) \cdot \beth_{q}(2 a+2 c) \beth_{q}(2 b+2 c)}{\Psi_{q}(a+b+2 c ; d+e) \Psi_{q}(a+c) \Psi_{q}(b+c) \cdot \beth_{q}(d) \beth_{q}(e) \beth_{q}(2 a+2 c+d) \beth_{q}(2 b+2 c+e)} .
\end{aligned}
$$

where $N=\left(\begin{array}{c}a+b+2 c+e \\ 3\end{array}\right)+d\left(\begin{array}{c}a+c \\ 2\end{array}\right)+\left(\begin{array}{c}a+c \\ 3\end{array}\right)-\left(\begin{array}{c}a+c+e \\ 3\end{array}\right)+b\left(\begin{array}{c}a+1 \\ 2\end{array}\right)+a\left(\begin{array}{c}b+1 \\ 2\end{array}\right)-a b(a+b+2 c+e)$.

Proof of Corollaries 4.3, 4.4, and 4.5. We "q-ify" the formula in corollaries 1.1, 1.2, and 1.3 respectively and calculate the corresponding power of $q$ in (26) to obtain the stated formula.

The rest of the section is devoted to the proof of Theorem 4.1 and Theorem 4.2.

\subsection{ProOF OF THE PRODUCT FORMULAS FOR SKEW SYT.}

Proof of Theorem 4.1. The starting point is showing that the skew shape $\lambda / b^{a}=$ $\Lambda(a, b, c, d, e, m)$ and the thick reverse hook $(b+c)^{a+c} / b^{a}=\Lambda(a, b, c, 0,0,0)$ have the same excited diagrams. To simplify the notation, let $R=(b+c)^{a+c}$ be the rectangle $[(a+c) \times(b+c)]$.

LEMmA 4.6. The skew shapes $\Lambda(a, b, c, d, e, m)$ and $\Lambda(a, b, c, 0,0,0)=R / b^{a}$ have the same excited diagrams.

Proof. This can be seen directly from the description of excited diagrams: by property (P1) from Section 4.1, the cell $(b, a)$ of $[\mu]$ cannot go past the cell $(b+c, a+c)$ so the rest of $[\mu]$ is confined in the rectangle $(b+c)^{a+c}$. Alternatively by Proposition 2.3, the excited diagrams of both shapes correspond to SSYT of shape $b^{a}$ with entries at most $b+c$. Then the map $\varphi^{-1}$ applied to such tableaux yields the same excited diagrams.

By (NHLF) and Lemma 4.6 we have:

$$
\frac{f^{\lambda / b^{a}}}{n !}=\left[\prod_{u \in[\lambda] \backslash R} \frac{1}{h_{\lambda}(i, j)}\right] \sum_{D \in \mathcal{E}\left(R / b^{a}\right)} \prod_{(i, j) \in R \backslash D} \frac{1}{h_{\lambda}(i, j)} .
$$


The sum over excited diagrams of $R / b^{a}$ with hook-lengths in $\lambda$ on the RHS above evaluates to a product.

LEMMA 4.7. For $\lambda$ and $R$ as above we have:

$$
\sum_{D \in \mathcal{E}\left(R / b^{a}\right)} \prod_{(i, j) \in R \backslash D} \frac{1}{h_{\lambda}(i, j)}=\frac{\Phi(a+b+c) \Phi(a) \Phi(b) \Phi(c)}{\Phi(a+b) \Phi(b+c) \Phi(a+c)} \prod_{(i, j) \in R / 0^{c} b^{a}} \frac{1}{h_{\lambda}(i, j)} .
$$

Proof. We write the sum of excited diagrams as an evaluation of $F_{(b+c)^{a+c} / b^{a}}(\mathbf{x} \mid \mathbf{y})$.

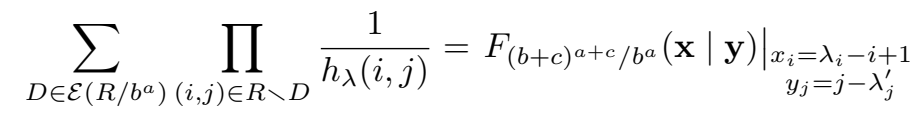

where $m=(b+c)(a+c)-b a$. Using Theorem 3.10 to obtain the symmetry of the series $F_{(b+c)^{a+c} / b^{a}}(\mathbf{x} \mid \mathbf{y})$ in $\mathbf{x}$ :

$$
\left.F_{(b+c)^{a+c} / b^{a}}(\mathbf{x} \mid \mathbf{y})\right|_{\substack{x_{i}=\lambda_{i}-i+1 \\ y_{j}=j-\lambda_{j}^{\prime}}}=\sum_{\Theta} \prod_{(i, j) \in \Theta} \frac{1}{h_{\lambda}(i, j)}
$$

where the sum is over tuples $\Theta:=\left(\theta_{1}, \ldots, \theta_{c}\right)$ of nonintersecting paths inside $(b+c)^{a+c}$ with endpoints $\theta_{p}:(p, 1) \rightarrow(a+p, b+c)$. Note that each tuple $\Theta$ has the same number of cells in each diagonal $i+j=k$. Also, by property (P2) of $\lambda$, the sum $\lambda_{i}+\lambda_{j}^{\prime}$ is constant when $i+j$ is constant. Thus each tuple $\Theta$ will have the same contribution to the sum on the RHS of (30), namely

$$
\prod_{(i, j) \in \Theta} \frac{1}{h_{\lambda}(i, j)}=\prod_{(i, j) \in R / 0^{c} b^{a}} \frac{1}{h_{\lambda}(i, j)}
$$

Lastly, the number of tuples $\Theta$ in (30) equals the number of excited diagrams $(b+$ $c)^{a+c} / b^{a}$, given by (2), see Example 2.5.

By Lemma 4.7, (27) becomes the following product formula for $f^{\lambda / b^{a}} / n$ ! equivalent to $(23)$ :

$$
\frac{f^{\lambda / b^{a}}}{n !}=\frac{\Phi(a+b+c) \Phi(a) \Phi(b) \Phi(c)}{\Phi(a+b) \Phi(b+c) \Phi(a+c)} \prod_{(i, j) \in \lambda / 0^{c} b^{a}} \frac{1}{h_{\lambda}(i, j)} .
$$

See Figure 5 for an illustration of the cells of $[\lambda]$ whose hook-lengths appear above. Finally, we carefully rewrite this product in terms of $\Psi(\cdot)$ and $\Psi^{(m)}(\cdot)$ to obtain (25).

\subsection{Proof of THE PRODUCT FORMUla FOR SKEW SSYT.}

Proof of Theorem 4.2. By (q-NHLF) and Lemma 4.6, we have:

$$
s_{\lambda / b^{a}}\left(1, q, q^{2}, \ldots\right)=\left[\prod_{(i, j) \in \lambda \backslash R} \frac{q^{\lambda_{j}^{\prime}-i}}{1-q^{h_{\lambda}(i, j)}}\right] \sum_{D \in \mathcal{E}\left(R / b^{a}\right)} \prod_{(i, j) \in R \backslash D} \frac{q^{\lambda_{j}^{\prime}-i}}{1-q^{h_{\lambda}(i, j)}}
$$

The sum over excited diagrams on the RHS evaluates to a product. We break the proof into two stages. 
LEmma 4.8. For $\lambda$ and $R$ as in the previous section, we have:

$$
\begin{aligned}
& \sum_{D \in \mathcal{E}\left(R / b^{a}\right)} \prod_{(i, j) \in R \backslash D} \frac{q^{\lambda_{j}^{\prime}-i}}{1-q^{h_{\lambda}(i, j)}} \\
& =q^{C\left(R / b^{a}\right)}\left[\prod_{(i, j) \in R / 0^{c} b^{a}} \frac{q^{a+c+e+m(b+c)}}{1-q^{h_{\lambda}(i, j)}}\right] \sum_{D \in \mathcal{E}^{\lambda}\left(R / 0^{c} b^{a}\right)} \prod_{(i, j) \in R \backslash D} q^{-j(m+1)},
\end{aligned}
$$

where $C(\lambda / \mu)$ is defined in (11).

Proof. We write the sum of excited diagrams as an evaluation of $F_{(b+c)^{a+c / b^{a}}}(\mathbf{x} \mid \mathbf{y})$.

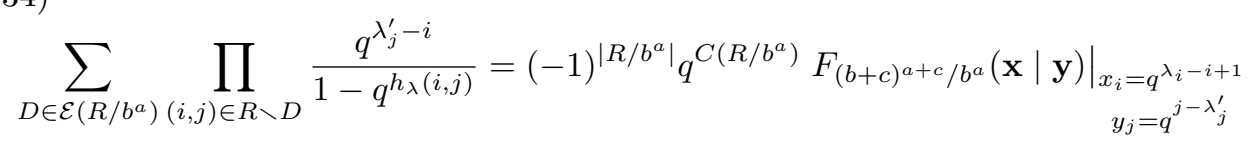

By Theorem 3.10, we have:

$$
\left.F_{(b+c)^{a+c / b^{a}}}(\mathbf{x} \mid \mathbf{y})\right|_{\substack{x_{i}=q^{\lambda_{i}-i+1} \\ y_{j}=q^{j-\lambda_{j}^{\prime}}}}=\sum_{\Theta}(-1)^{\left|R / b^{a}\right|} \prod_{(i, j) \in \Theta} \frac{q^{\lambda_{j}^{\prime}-j}}{1-q^{h_{\lambda}(i, j)}} .
$$

Each tuple $\Theta$ has the same number of cells in each diagonal $i+j=k$. Also by property (P2) of $\lambda$, the sum $\lambda_{i}+\lambda_{j}^{\prime}$ is constant when $i+j$ is constant. Thus each term in the sum corresponding to a tuple $\Theta$ has the same denominator. Factoring this contribution out of the sum and using $\lambda_{j}^{\prime}=a+c+e+m(b+c-j)$, gives:

$$
\begin{aligned}
\sum_{D \in \mathcal{E}\left(R / b^{a}\right)} \prod_{(i, j) \in R \backslash D} \frac{q^{\lambda_{j}^{\prime}-i}}{1-q^{h_{\lambda}(i, j)}} & \\
& =q^{C\left(R / b^{a}\right)}\left[\prod_{(i, j) \in R / 0^{c} b^{a}} \frac{q^{a+c+e+m(b+c)}}{1-q^{h_{\lambda}(i, j)}}\right] \sum_{\Theta} \prod_{(i, j) \in \Theta} q^{-j(m+1)} .
\end{aligned}
$$

Finally, we rewrite the sum over tuples $\Theta$ as a sum over NE-excited diagrams $\mathcal{E}^{\nearrow}\left(R / 0^{c} b^{a}\right)$, see Section 3.4.

Next, we prove that the sum over NE-excited diagrams on the RHS of (33) also factors.

LEMma 4.9. In the notation above, we have:

$$
\sum_{D \in \mathcal{E}^{\nearrow}\left(R / 0^{c} b^{a}\right)} \prod_{(i, j) \in R \backslash D} q^{-j(m+1)}=q^{-N_{2}} \prod_{i=1}^{a} \prod_{j=1}^{b} \prod_{k=1}^{c} \frac{1-q^{(m+1)(i+j+k-1)}}{1-q^{(m+1)(i+j+k-2)}},
$$

where $N_{2}=(m+1)\left((a+c)\left(\begin{array}{c}b+c+1 \\ 2\end{array}\right)-a\left(\begin{array}{c}b+1 \\ 2\end{array}\right)\right)$.

Proof. We factor out a power of $q^{-N_{1}}$ where $N_{1}=\sum_{(i, j) \in R} j(m+1)$, so that the weight of each excited diagram $D$ is $\prod_{(i, j) \in D} q^{j(m+1)}$. We have:

$$
\sum_{D \in \mathcal{E}^{\gamma}\left(R / 0^{c} b^{a}\right)} \prod_{(i, j) \in R \backslash D} q^{-j(m+1)}=q^{-N_{1}} \sum_{D \in \mathcal{E}^{\lambda}\left(R / 0^{c} b^{a}\right)} \prod_{(i, j) \in D} q^{j(m+1)} .
$$


Reflecting by the diagonal, this sum equals the sum over excited diagrams $\mathcal{E}\left(R^{\prime} / a^{b}\right)$ where $R^{\prime}=(a+c)^{b+c}$. We then use (12) in Example 3.3, with $q \leftarrow q^{m+1}$, to obtain:

$$
\begin{aligned}
\sum_{D \in \mathcal{E}^{\lambda}\left(R / 0^{c} b^{a}\right)} \prod_{(i, j) \in R \backslash D} q^{-j(m+1)} & =q^{-N_{1}} \sum_{D \in \mathcal{E}\left(R^{\prime} / a^{b}\right)} \prod_{(i, j) \in D} q^{i(m+1)}, \\
& =q^{-N_{1}+(m+1) a\left(\begin{array}{c}
b+1 \\
2
\end{array}\right)} \prod_{i=1}^{a} \prod_{j=1}^{b} \prod_{k=1}^{c} \frac{1-q^{(m+1)(i+j+k-1)}}{1-q^{(m+1)(i+j+k-2)}},
\end{aligned}
$$

as desired.

Combining Lemmas 4.8 and 4.9, we obtain:

$$
\begin{aligned}
& \sum_{D \in \mathcal{E}\left(R / b^{a}\right)} \prod_{(i, j) \in R \backslash D} \frac{q^{\lambda_{j}^{\prime}-i}}{1-q^{h_{\lambda}(i, j)}} \\
= & q^{C\left(R / b^{a}\right)-N_{2}}\left(\prod_{(i, j) \in R / 0^{c} b^{a}} \frac{q^{a+c+e+m(b+c)}}{1-q^{h_{\lambda}(i, j)}}\right) \prod_{i=1}^{a} \prod_{j=1}^{b} \prod_{k=1}^{c} \frac{1-q^{(m+1)(i+j+k-1)}}{1-q^{(m+1)(i+j+k-2)}} .
\end{aligned}
$$

Next we find a simpler expression for the power of $q$ above.

Proposition 4.10. The power of $q$ on the RHS of (32) is equal to

$$
\sum_{(i, j) \in R / b^{a}}\left(\lambda_{j}^{\prime}-i\right) .
$$

Proof. The term $N_{2}$ in the power of $q$ can be written as $N_{2}=\sum_{(i, j) \in R / b^{a}}(m+1) j$. Using this, we have:

$$
C\left(R / b^{a}\right)-N_{2}=\sum_{(i, j) \in R / b^{a}}(-i-m j) .
$$

Since $R / 0^{c} b^{a}$ has the same number of cells as $R / b^{a}$ and $\lambda_{j}^{\prime}=a+c+e+m(b+c-j)$, then

$$
C\left(R / b^{a}\right)-N_{2}+(a+c+e+m(b+c))\left|R / 0^{c} b^{a}\right|=\sum_{(i, j) \in R / b^{a}}\left(\lambda_{j}^{\prime}-i\right),
$$

is the desired degree in the RHS of (32).

Finally, Theorem 4.2 follows by substituting (36) in the RHS of (32), simplifying the power of $q$ with Proposition 4.10, and collecting the other powers of $q$ from the cells $(i, j)$ in $\lambda \backslash R$.

\section{Excited Diagrams and Schubert POLYNomials}

In this section we obtain a number of product formulas for principal evaluations of Schubert polynomials for two permutation families: vexillary and 321-avoiding permutations.

5.1. Vexillary permutations. Recall from $\S 2.2$ that to a vexillary permutation $w$ we associate a shape $\mu(w)$ contained in a supershape $\lambda(w)$. A formula for the double Schubert polynomial $\mathfrak{S}_{w}(\mathbf{x} ; \mathbf{y})$ of a vexillary permutation in terms of excited diagrams of the skew shape $\lambda(w) / \mu(w)$ is given in [31]. This formula was already known in terms of flagged tableaux [78] (see Section 2.7), and in terms of flagged Schur functions [45, 44]. 
THEOREM 5.1 (Wachs [78], Knutson-Miller-Yong [31]). Let $w$ be a vexillary permutation of shape $\mu$ and supershape $\lambda$. Then the double Schubert polynomial of $w$ is equal to

$$
\mathfrak{S}_{w}(\mathbf{x} ; \mathbf{y})=\sum_{D \in \mathcal{E}(\lambda / \mu)} \prod_{(i, j) \in D}\left(x_{i}-y_{j}\right) .
$$

EXAMPLE 5.2. For the permutation $w=1432$, we have the shape $\mu=21$ and the supershape $\lambda=332$ :

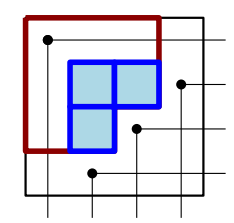

There are five excited diagrams in $\mathcal{E}(332 / 21)$ (see Example 2.2), and so

$$
\begin{aligned}
\mathfrak{S}_{1432}(\mathbf{x} ; \mathbf{y})=\left(x_{1}-y_{1}\right)\left(x_{1}-y_{2}\right)\left(x_{2}-y_{1}\right)+\left(x_{1}-y_{1}\right)\left(x_{1}-y_{2}\right)\left(x_{3}-y_{2}\right) \\
+\left(x_{1}-y_{1}\right)\left(x_{2}-y_{3}\right)\left(x_{2}-y_{1}\right)+\left(x_{1}-y_{1}\right)\left(x_{2}-y_{3}\right)\left(x_{3}-y_{2}\right) \\
+\left(x_{2}-y_{2}\right)\left(x_{2}-y_{3}\right)\left(x_{3}-y_{2}\right) .
\end{aligned}
$$

We have seen that he multivariate sum over excited diagrams on the RHS of (37) is also an evaluation of a factorial Schur function.

COROLlaRY 5.3. Let $w$ be a vexillary permutation of shape $\mu$ and supershape $\lambda$, such that $\lambda / \mu \subset d \times(m-d)$ for some $d, m$. Then:

$$
\mathfrak{S}_{w}(\mathbf{x} ; \mathbf{y})=s_{\mu}^{(d)}\left(\mathbf{x} \mid \mathbf{z}^{\langle\lambda\rangle}\right) .
$$

Proof. By (37), we have: $\mathfrak{S}_{w}(\mathbf{x} ; \mathbf{y})=G_{\lambda / \mu}(\mathbf{x} \mid \mathbf{y})$. By Lemma 3.4, this is given by an evaluation of a factorial Schur function.

Combining this result with the Macdonald identity (8) for single Schubert polynomials gives the following identity for the principal evaluation $\Upsilon_{w}$ of the Schubert polynomial.

THEOREM 5.4. Let $w$ be a vexillary permutation of shape $\mu$ and supershape $\lambda$. Then:

$$
\Upsilon_{w}=|\mathcal{E}(\lambda / \mu)|
$$

Proof. This follows directly from (37) by setting $x_{i}=1$ and $y_{i}=0$ for all $i$, and from the Macdonald identity (8).

EXAMPLE 5.5. Continuing the previous Example 5.2, the reduced words for $w=1432$ are $(2,3,2)$ and $(3,2,3)$. We indeed have:

$$
\Upsilon_{1432}=\mathfrak{S}_{1432}(1,1,1)=\frac{1}{3 !}(2 \cdot 3 \cdot 2+3 \cdot 2 \cdot 3)=5=|\mathcal{E}(332 / 21)|
$$

Theorem 5.4 generalizes an identity in [17, Thm. 2.1] from dominant permutations (avoiding 132) to vexillary permutations. To state their result we need the following notation. Given a partition $\mu$ and $c \in \mathbb{N}$, let $R P P_{\mu}(c)$ be the number of reverse plane partitions of shape $\mu$ with entries $\leqslant c$. Let $1^{c} \diamond \mu=\left(\mu_{1}+c\right)^{c}\left(c+\mu_{1}\right)\left(c+\mu_{2}\right) \ldots$

Proposition 5.6. For the shape $\mu$ of a dominant permutation, we have:

$$
\left|\operatorname{RPP}_{\mu}(c)\right|=\left|\mathcal{E}\left(\left(1^{c} \diamond \mu\right) / \mu\right)\right| .
$$

Proof. By Proposition 2.3, the RHS is equal to the number of SSYT of shape $\mu$ with entries in row $i$ at most $c+i$. By subtracting $i$ from the entries in row $i$, such SSYT are in correspondence with RPP of shape $\mu$ with entries $\leqslant c$. 
For the rest of the section we will use the following notation for the principal evaluation:

$$
\Upsilon_{w}(c):=\Upsilon_{1^{c} \times w}=\frac{1}{\ell(w) !} \sum_{\left(r_{1}, \ldots, r_{\ell}\right) \in R(w)}\left(c+r_{1}\right) \cdots\left(c+r_{\ell}\right) .
$$

COROllary 5.7 (Fomin-Kirillov [17]). For a dominant permutation $w$ of shape $\mu$ we have:

$$
\Upsilon_{w}(c)=\left|\operatorname{RPP}_{\mu}(c)\right|
$$

Proof. The permutation $1^{c} \times w=\left(1,2, \ldots, c, c+w_{1}, c+w_{2}, \ldots\right)$ is a vexillary permutation of shape $\mu$ and supershape $\lambda=1^{c} \diamond \mu$. Also, the reduced words of $1^{c} \times w$ are of the form $\left(c+r_{1}, \ldots, c+r_{\ell}\right)$, where $\left(r_{1}, \ldots, r_{\ell}\right)$ is a reduced word of $w$. We then apply Theorem 5.4 and Proposition 5.6 to obtain the result.

5.2. Product formulas for Macdonald type Sums. As special cases of Theorem 5.4 we obtain two identities from [17] for two families of dominant permutations, followed by new identities for families of vexillary permutation. See Figure 6 for illustrations of some of these families.

Corollary 5.8 (staircase [17]). For the permutation $w_{0}=n \ldots 21$, we have:

$$
\Upsilon_{w_{0}}(c)=\frac{\Phi(2 c+2 n-1) \Phi(n) \cdot \beth(2 c+1) \beth(2 n-1)}{\Phi(n+2 c) \Phi(2 n-1) \cdot \beth(2 c+2 n-1)} .
$$

Proof. The longest element $w_{0}$ is the dominant permutation with shape $\mu=\delta_{n}:=$ $(n-1, \ldots, 2,1)$. The result follows by Corollary 5.7 and Proctor's formula [63]:

$$
\Upsilon_{w_{0}}(c)=\left|\operatorname{RPP}_{\delta_{n}}(c)\right|=\prod_{1 \leqslant i<j \leqslant n} \frac{2 c+i+j-1}{i+j-1},
$$

written in terms of superfactorials.

Note that the case $c=1$ above gives $\Upsilon_{w_{0}}(1)=\frac{1}{n+1}\left(\begin{array}{c}2 n \\ n\end{array}\right)$; see [80] for several proofs of this case.

Corollary 5.9 (box formula [17]). Consider the permutation $u(a, b)$ defined as

$$
u(a, b):=b(b+1) \cdots(a+b) 12 \ldots(b-1) .
$$

Then we have:

$$
\Upsilon_{u(a, b)}(c)=\frac{\Phi(a+b+c) \Phi(a) \Phi(b) \Phi(c)}{\Phi(a+b) \Phi(b+c) \Phi(a+c)} .
$$

Proof. The permutation $u(a, b)$ is a dominant permutation with shape $b^{a}$. The result follows by Corollary 5.7 and the MacMahon box formula (2).

For the rest of this subsection, we consider examples that are vexillary but not dominant. These results partially answer a question in [7, Open Problem 2]. First, we give a family of permutations $z(a)$ with principal evaluation given by a power of 2 .

Corollary 5.10. Consider the permutation $z(a):=135 \ldots(2 a-1) 246 \cdots(2 a)$. Then we have

$$
\Upsilon_{z(a)}=2^{\left(\begin{array}{c}
a \\
2
\end{array}\right)}
$$

Proof. The vexillary (actually, Grassmannian) permutation $z(a)$ has shape $\mu=\delta_{a}$ and supershape $\lambda=(2 a-2)^{a}$. By Proposition 2.3, the number of excited diagrams 
equals the number of SSYT of shape $\mu$ with entries at most $a$. This number is given by the hook-content formula

$$
s_{\delta_{a}}\left(1^{a}\right)=\prod_{(i, j) \in\left[\delta_{a}\right]} \frac{a+j-i}{h_{\delta_{a}}(i, j)}=\prod_{i=1}^{a-1} \prod_{j=1}^{a-i} \frac{a+j-i}{2(a-i-j)+1} .
$$

A direct calculation gives the desired formula (see e.g. [53, Prop. 10.3]).

Second, we restate Corollary 1.4 as follows:

Corollary $5.11\left(2413 \otimes 1^{a}\right.$ case). Consider the permutation $v(a):=2413 \otimes 1^{a}$. Then, for all $c \geqslant a$, we have:

$$
\Upsilon_{v(a)}(c)=\frac{\Phi(4 a+c) \Phi(c) \Phi(a)^{4} \Phi(3 a)^{2}}{\Phi(3 a+c) \Phi(a+c) \Phi(2 a)^{2} \Phi(4 a)} .
$$

Proof. The vexillary permutation $1^{c} \times v(a)$ has length $3 a^{2}$, shape $\mu=(2 a)^{a} a^{a}$ and supershape $\lambda=(c+3 a)^{c+2 a}$. The reduced words of $1^{c} \times v(a)$ are obtained from those of $v(a)$ after shifting by $c$. By Theorem 5.4 for $1^{c} \times v(a)$, we have:

$$
\Upsilon_{v(a)}(c)=|\mathcal{E}(\lambda / \mu)| \text {. }
$$

By Proposition 2.3, the number of excited diagrams equals the number of SSYT of shape $\mu$ with entries at most $2 a+c$. This number is given by the hook-content formula

$$
s_{\mu}\left(1^{2 a+c}\right)=\prod_{(i, j) \in[\mu]} \frac{2 a+c+j-i}{h_{\mu}(i, j)} .
$$

This product can be written in terms of superfactorials as stated.

Next, we consider whether the skew shapes in the first part of the paper come from vexillary permutations. We failed to obtain the skew shape $\Lambda(a, b, c, d, e, 0)$ this way, but the next vexillary permutation yields a shape similar to $\Lambda(a, a, c, a, a, 0)$.

COROLlary 5.12. For the vexillary permutation

$$
w(a):=(a+1, a+2, \ldots, 2 a-1,2 a+1,1,2, \ldots, a-1,2 a, a) .
$$

we have:

$$
\Upsilon_{w(a)}(c)=\frac{\Phi(2 a+c) \Phi(a)^{2} \Phi(c)}{\Phi(a+c)^{2} \Phi(2 a-1)}\left[\frac{a(2 a+c)\left(2 a c+4 a^{2}-1\right)}{2\left(4 a^{2}-1\right)}\right] .
$$

Proof. The vexillary permutation $1^{c} \times w(a)$ has length $2+a^{2}$, shape $\mu=(a+1) a^{a-1} 1$ and supershape $\lambda=(2 a+c)^{c+a}(a+c)^{a}$, see Figure 6 . The reduced words of $1^{c} \times w(a)$ are obtained from those of $w(a)$ by shifting by $c$. By Theorem 5.4 for $1^{c} \times w(a)$, we have:

$$
\Upsilon_{w(a)}(c)=|\mathcal{E}(\lambda / \mu)|
$$

By Proposition 2.3, the number of excited diagrams of shape $\lambda / \mu$ is equal to the number of SSYT of shape $\mu$ with entries in the top $a$ rows at most $a+c$ and the single box in the $a+1$ row at most $2 a+c$. Depending on the value of this single box, whether it is at most $a+c$ or between $a+c+1$ and $2 a+c$, this number equals the sum of two specializations of Schur functions:

$$
|\mathcal{E}(\lambda / \mu)|=s_{(\nu, 1)}\left(1^{a+c}\right)+a \cdot s_{\nu}\left(1^{a+c}\right),
$$

where $\nu=(a+1) a^{a-1}$. Using the hook-content formula, this number can be written in terms of superfactorials as in the corollary. 

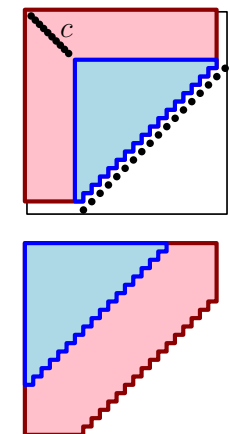

(a)
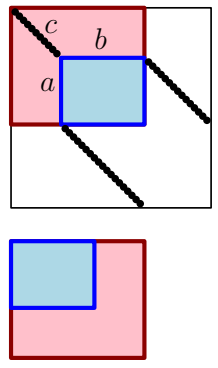

(b)
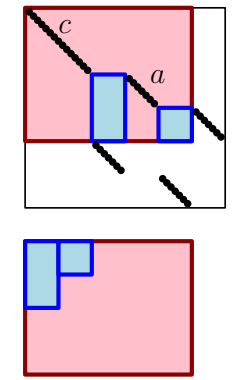

(c)
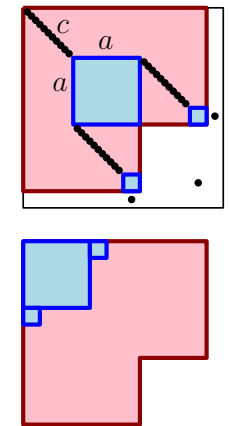

(d)
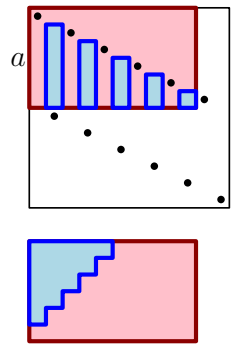

(e)

Figure 6. The diagram (top) and skew shape (bottom) of the vexillary permutations $1^{c} \times w$ where $w=w_{0}, u(a, b), v(a), w(a)$, and $z(a)$ respectively.

5.3. 321-AVOIding PERmutations. Recall from Section 2.2 that the diagram of a 321-avoiding permutation is, up to removing empty rows and columns and flipping columns, the diagram of a skew shape $\lambda / \mu$. By Theorem 2.1 (stating [8, Prop 2.2]) we can realize every skew shape $\lambda / \mu$ as the diagram of the 321-avoiding permutation given by the reduced word $\mathrm{rw}(\lambda / \mu)$. The map from shapes to permutations is outlined in Section 2.2.

THEOREM 5.13. Let $w$ be a 321-avoiding permutation. Then its diagram gives a skew shape $\lambda / \mu$. Conversely, every skew shape $\lambda / \mu$ can be realized from the diagram of a 321-avoiding permutation $w$. In both cases, we have:

$$
\Upsilon_{w}=\frac{1}{\ell !} r_{1} \cdots r_{\ell} f^{\lambda / \mu},
$$

where $\ell=|\lambda / \mu|$ and $\left(r_{1}, \ldots, r_{\ell}\right)$ is a reduced word of $w$.

Proof. The fact that diagrams of 321-avoiding permutations yield skew shapes and its converse are explained in Section 2.2.

Assume that the 321 -avoiding permutation has skew shape $\operatorname{skewsh}(w)=\lambda / \mu$. The reduced words of a 321-avoiding permutation are obtained from one another by only using commutation relations $s_{i} s_{j}=s_{j} s_{i}$ for $|i-j|>1$ [8, Thm. 2.1]. Thus, all reduced words $\left(r_{1}, \ldots, r_{\ell}\right)$ of $w$ have the same product $r_{1} \cdots r_{\ell}$. Also, the number of reduced words of $w$ equals $f^{\lambda / \mu}$, see $[8$, Cor. 2.1]. The result then follows by using these two facts and Macdonald's identity (8).

As an illustration we obtain permutations such that $\Upsilon_{w}$ give double factorials and Euler numbers.

Corollary 5.14. For the permutations $w=2143 \cdots(2 n)(2 n-1)$ and $w \otimes 1^{a}$, we have:

$$
\Upsilon_{w}=(2 n-1) ! ! \quad \text { and } \quad \Upsilon_{w \otimes 1^{a}}=\frac{\Phi(2 n a) \Phi(a)^{2 n-2}}{\Phi(2 a)^{n}}\left[\prod_{k=1}^{n-1} \frac{\Phi(2 k a)}{\Phi((2 k+1) a)}\right]^{2} .
$$

Proof. The number of SYT of the diagonal shape $\delta_{n+1} / \delta_{n}$ is $n$ !. By the construction from Theorem 2.1, from this shape we read off the reduced word

$$
\operatorname{rw}\left(\delta_{n+1} / \delta_{n}\right)=(1,3,5, \ldots, 2 n-1),
$$

defining the permutation $w$. See Figure 8(a) for an example. The product of the entries of this reduced word is $(2 n-1)$ !! The result then follows by Theorem 5.13 . The second 
formula comes from the 321-avoiding permutation $w \otimes 1^{a}$ whose skew shape consists of $n$ disjoint $a \times a$ blocks.

Let $\operatorname{Alt}(n)=\{\sigma(1)<\sigma(2)>\sigma(3)<\sigma(4)>\ldots\} \subset \mathbb{S}_{n}$ be the set of alternating permutations. The number $E_{n}=|\operatorname{Alt}(n)|$ is the $n$-th Euler number (see [70, A000111]), with the generating function

$$
\sum_{n=0}^{\infty} E_{n} \frac{x^{n}}{n !}=\tan (x)+\sec (x) .
$$

Let $x(n)$ be a permutation with reduced word corresponding to the zigzag shape

$$
\operatorname{rw}\left(\delta_{n+2} / \delta_{n}\right)=(2,1,4,3, \ldots, 2 n, 2 n-1,2 n+1) .
$$

Similarly, define $y(n)$ and $z(n)$ to be the permutations with reduced words corresponding to shapes $(n+1)^{2} n(n-1) \ldots 2 / \delta_{n}$ and $(n+2)^{3}(n+1) n \ldots 3 / \delta_{n}$, respectively.

Corollary 5.15. For the permutations $x(n), y(n)$, and $z(n)$ defined above, we have:

$$
\Upsilon_{x(n)}=E_{2 n+1}, \quad \Upsilon_{y(n)}=\frac{n ! E_{2 n+1}}{2^{n}}, \quad \Upsilon_{z(n)}=\frac{(n+1)(2 n+3) ! E_{2 n+1}^{2}}{n ! 2^{5 n+1}\left(2^{2 n+2}-1\right)}
$$

Proof. The number of SYT of the zigzag shape $\delta_{n+2} / \delta_{n}$ is given by the Euler number $E_{2 n+1}$. By the construction from Theorem 2.1, from this shape we read off the reduced word

$$
\operatorname{rw}\left(\delta_{n+2} / \delta_{n}\right)=(2,1,4,3, \ldots, 2 n, 2 n-1,2 n+1),
$$

defining the permutation $x(n)$. The product of the entries of this reduced word is $(2 n+1)$ ! The first equality then follows by Theorem 5.13.

The second and third equalities follow by a similar argument for the 3-zigzag and 5 -zigzag shape, respectively, whose number of SYT is given by [5, Thm. 1]. We omit the easy details. See Figure 8(b), 8(c) and 8(d) for examples.

We also obtain a family of 321-avoiding permutations $w$ that yield the skew shapes from Section 4 with product $\Lambda(a, b, c, d, e, f)$. Then by theorems 4.1 and 5.13 , for such permutations, $\Upsilon_{w}$ is given by a product formula. We illustrate this for the cases $\Lambda(a, a, a, a, a, 0)$ and $\Lambda(a, a, a, 1,1,1)$. See Figure $8(\mathrm{e}), 8(\mathrm{f})$ for examples.

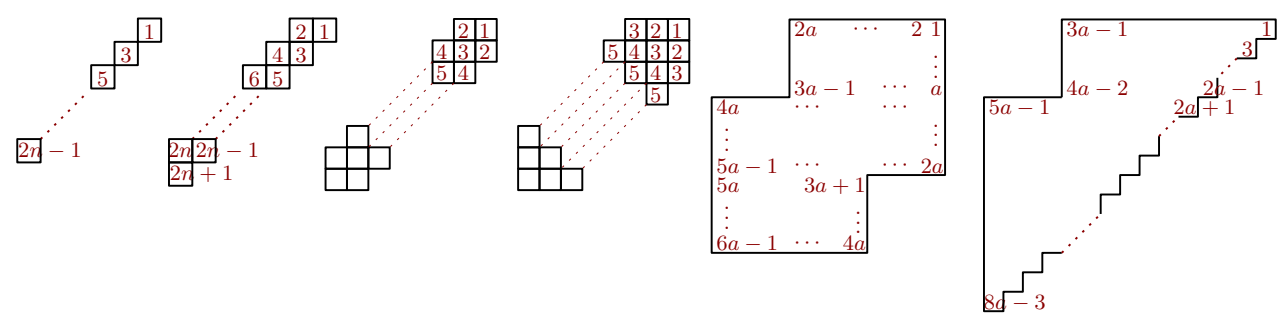

FiguRE 7. The reduced words $\operatorname{rw}(\lambda / \mu)$ of the skew shapes $\delta_{n+1} / \delta_{n}$, the zigzag $\delta_{n+2} / \delta_{n}$, 3-zigzag, 5-zigzag, $(3 a)^{2 a}(2 a)^{a} / a^{a}$, and $\delta_{4 a} / a^{a}$.

We now restate Corollary 1.5 in the notation above.

Corollary 5.16 (shape $\left.(3 a)^{2 a}(2 a)^{a} / a^{a}\right)$. For the permutation $s(a):=351624 \otimes 1^{a}$, we have:

$$
\Upsilon_{s(a)}=\frac{\Phi(a)^{5} \Phi(3 a)^{2} \Phi(5 a)}{\Phi(2 a)^{4} \Phi(4 a)^{2}}
$$


Proof. The reading word associated to the shape $3^{2} 2 / 1$ is $(2,1,4,3,2,5,4)$ which defines the permutation 351624. Similarly, the shape $(3 a)^{2 a}(2 a)^{a} / a^{a}$ yields a reduced word $\left(r_{1}, \ldots, r_{7 a^{2}}\right)$, defining the 321-avoiding permutation $s(a)=351624 \otimes 1^{a}$. By Theorem 5.13, we have:

$$
\Upsilon_{s(a)}=\frac{r_{1} \cdots r_{7 a^{2}}}{\left(7 a^{2}\right) !} f^{(3 a)^{2 a}(2 a)^{a} / a^{a}}
$$

The result now follows by writing the product of the entries of the reduced word as

$$
r_{1} \cdots r_{7 a^{2}}=\frac{\Phi(3 a)^{2} \Phi(6 a)}{\Phi(2 a)^{2} \Phi(4 a)^{2}}
$$

(see Figure 7). Now use Corollary 1.1 to write the number of SYT as

$$
f^{(3 a)^{2 a}(2 a)^{a} / a^{a}}=\frac{\left(7 a^{2}\right) ! \Phi(a)^{5} \Phi(5 a)}{\Phi(2 a)^{2} \Phi(6 a)},
$$

and the result follows.

COROLlaRY 5.17 (shape $\left.\delta_{4 a} / a^{a}\right)$. Let $t(a)$ be the permutation of size $(8 a-2)$ obtained from the reading word of the skew shape $\delta_{4 a} / a^{a}$. Then:

$$
\Upsilon_{t(a)}=\frac{\Phi(a)^{3} \Phi(3 a) \Phi(4 a-1) \Phi(8 a-2) \cdot \Psi(a) \Psi(3 a)}{\Phi(2 a)^{2} \Phi(3 a-1) \Phi(5 a-1) \cdot \Psi(2 a)^{2} \Psi(4 a) \cdot \beth(8 a-2)} .
$$

Proof. The reduced word $\operatorname{rw}\left(\delta_{4 a} / a^{a}\right)$ defines the permutation $t(a)$. By Theorem 5.13 we have:

$$
\Upsilon_{t(a)}=\frac{r_{1} \cdots r_{\ell}}{\ell !} f^{\delta_{4 a+1} / a^{a}}
$$

We can write the product of the entries of the reduced word as

$$
r_{1} r_{2} \cdots r_{\ell}=\frac{\Phi(4 a-1) \Phi(8 a-2)}{\Phi(3 a-1) \Phi(5 a-1)\rfloor(8 a-2)},
$$

(see Figure 7). On the other hand, Corollary 1.2 gives:

$$
f^{\delta_{4 a+1} / a^{a}}=\frac{\ell ! \cdot \Phi(a)^{3} \Phi(3 a) \Psi(a) \Psi(3 a)}{\Phi(2 a)^{2} \Psi(2 a)^{2} \Psi(4 a)},
$$

where $\ell=\left(\begin{array}{c}4 a \\ 2\end{array}\right)-a^{2}$. Combining these formulas, we obtain the result.

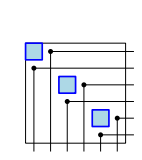

(a)

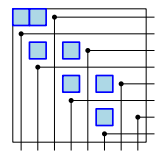

(b)

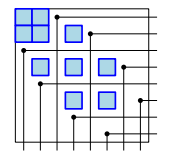

(c)

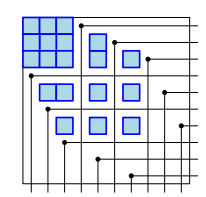

(d)

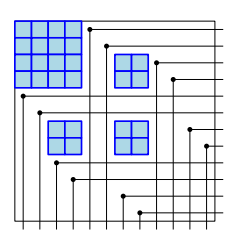

(e)

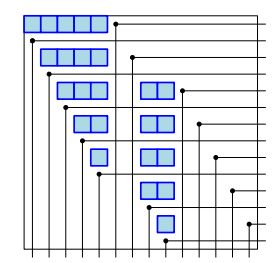

(f)

Figure 8. The diagram of the 321-avoiding permutations $w=$ $214365, x(3)=31527486, y(3), z(3), s(2)=351624 \otimes 1^{2}$ and $t(2)$, with skew shapes $\delta_{4} / \delta_{3}, \delta_{5} / \delta_{3}, 4^{2} 32 / \delta_{3}, 5^{3} 43 / \delta_{3}, 6^{4} 4^{2} / 2^{2}$ and $\delta_{8} / 2^{2}$ respectively. 
5.4. Conjectural Formula. The number of SYT of the skew shape of the vexillary permutation $1^{c} \times w(a)$ defined in (39) appears to have the following formula similar to Corollary 1.1 when $a=b=d=e$.

Conjecture 5.18 (joint with C. Krattenthaler). Let $\lambda=(2 a+c)^{c+a}(a+c)^{a}, \mu=$ $(a+1) a^{a-1} 1$. Then:

$$
f^{\lambda / \mu}=n ! \frac{\Phi(a)^{4} \Phi(c) \Phi(4 a+c)}{\Phi(2 a)^{2} \Phi(4 a+2 c)}\left[\frac{a^{2}\left(\left(2 a^{2}+4 a c+c^{2}\right)^{2}-a^{2}\right)}{4 a^{2}-1}\right] .
$$

where $n=|\lambda / \mu|=(2 a+c)^{2}-2 a^{2}-2$.

REMARK 5.19. For $a=c$, formula (43) for the number of SYT of shape $(3 a)^{2 a}(2 a)^{a} /(a+$ 1) $a^{a-1} 1$ is

$$
f^{\lambda / \mu}=n ! \frac{\Phi(a)^{5} \Phi(5 a)}{\Phi(2 a)^{2} \Phi(6 a)}\left[\frac{\left(49 a^{2}-1\right) a^{4}}{4 a^{2}-1}\right] .
$$

This formula was suggested by Christian Krattenthaler, ${ }^{(4)}$ based on computational data and was a precursor of the conjecture above. ${ }^{(5)}$ Note the close resemblance of (44) and (41), which are the same up to a polynomial factor. This suggests that perhaps there is a common generalization.

\section{Asymptotic applichtions}

6.1. NUmber OF SYT. In [53], we prove that for a sequence $\bar{\pi}=\left\{\pi^{(n)}\right\}$ of strongly stable skew shapes $\pi^{(n)}=\lambda^{(n)} / \mu^{(n)},\left|\pi^{(n)}\right|=n$, we have:

$$
\log \left|\operatorname{SYT}\left(\pi^{(n)}\right)\right|=\frac{1}{2} n \log n+O(n) .
$$

Furthermore, we conjecture that

$$
\log \left|\operatorname{SYT}\left(\pi^{(n)}\right)\right|=\frac{1}{2} n \log n+c n+o(n),
$$

for some constant $c=c(\bar{\pi}){ }^{(6)}$ Here by the strongly stable skew shape we mean a sharp convergence to the limit shape of the Young diagrams of $\pi^{(n)}$ under scaling $1 / \sqrt{n}$, as $n \rightarrow \infty$; we refer to [53] for details. ${ }^{(7)}$

Until this paper, the exact value of $c(\bar{\pi})$ was possible to compute only for the usual and shifted shapes. Here we have a new family of shapes where this is possible.

Theorem 6.1. Fix $\alpha, \beta, \gamma, \delta, \epsilon \geqslant 0, m \in \mathbb{N}$, and let

$$
\pi^{(n)}=\Lambda(\lfloor\alpha n\rfloor,\lfloor\beta n\rfloor,\lfloor\gamma n\rfloor,\lfloor\delta n\rfloor,\lfloor\epsilon n\rfloor, m) .
$$

Then the asymptotic formula $(* *)$ holds for some $c=c(\alpha, \beta, \gamma, \delta, \epsilon, m)$.

The proof of the theorem is straightforward from the product formula in Theorem 4.1 and asymptotic formulas in $\S 2.10$. We omit the details.

\footnotetext{
${ }^{(4)}$ Personal communication.

${ }^{(5)}$ Both Krattenthaler's formula and Conjecture 5.18 were recently established in [29].

${ }^{(6)}$ This conjecture was recently established in [56]

${ }^{(7)}$ See [14] for related results for other growth regimes.
} 
EXAMPLE 6.2 . Let $\pi=\Lambda(a, a, a, a, a, 0)$. Then $|\pi|=7 a^{2}$ and by (41), we have:

$$
\begin{aligned}
\log f^{\pi}= & \log \frac{\left(7 a^{2}\right) ! \Phi(a)^{5} \Phi(5 a)}{\Phi(2 a)^{2} \Phi(6 a)}=7 a^{2} \log a \\
& +\left(\frac{7}{2}-22 \log 2-18 \log 3+\frac{25}{2} \log 5+7 \log 7\right) a^{2}+O(a \log a)
\end{aligned}
$$

The sum in parentheses shifted by $(7 / 2) \log 7$ is the exact value of the constant $c(1,1,1,1,1,0)$ as in the theorem.

6.2. Principal Schubert Evaluations. In recent years, there has been some interest in the asymptotics of the principle evaluation $\Upsilon_{w}=\mathfrak{S}_{w}(1, \ldots, 1)$. Notably, Stanley [73] defined

and observed that

$$
u(n):=\max _{w \in S_{n}} \Upsilon_{w}
$$

$$
\frac{1}{4} \leqslant \liminf _{n \rightarrow \infty} \frac{\log _{2} u(n)}{n^{2}} \leqslant \limsup _{n \rightarrow \infty} \frac{\log _{2} u(n)}{n^{2}} \leqslant \frac{1}{2} .
$$

Stanley also suggested existence of the limit of $\frac{1}{n^{2}} \log _{2} u(n)$, and that it is achieved on a certain "limit shape". Below we apply our product formulas to obtain asymptotics of $\Upsilon_{w}$ for some families of $w$.

Proposition 6.3 (zigzag permutations). For permutations $w \in S_{2 n}$ as in Corollary 5.14, $x(n), y(n) \in S_{2 n+2}$, and $z(n) \in S_{2 n+4}$ as in Corollary 5.15, we have: $\log \Upsilon_{w}=\Theta(n \log n), \log \Upsilon_{x(n)}=\Theta(n \log n), \log \Upsilon_{y(n)}=\Theta(n \log n)$, and $\log \Upsilon_{z(n)}=$ $\Theta(n \log n)$.

The proof follows immediately from the product formulas in corollaries as above, the asymptotics of $(2 n-1)$ !! and of the Euler numbers:

$$
E_{n} \sim n !\left(\frac{2}{\pi}\right)^{n} \frac{4}{\pi}(1+o(1)) \text { as } n \rightarrow \infty,
$$

(see e.g. [16, 73]).

Proposition 6.4 (Macdonald permutations). Consider permutations $w_{0} \in S_{2 k}, \widehat{w}_{0}=$ $1^{k} \times w_{0} \in S_{3 k}$ as in Corollary 5.8, $u(k, k) \in S_{2 k}, \widehat{u}(k, k)=1^{k} \times u(k, k) \in S_{3 k}$ as in Corollary 5.9. Then we have:

$$
\log \Upsilon_{\widehat{w}_{0}} \sim 2(\log C) k^{2}, \quad \text { and } \quad \log \Upsilon_{\widehat{u}(k, k)} \sim(\log C) k^{2}, \quad \text { where } \quad C=\frac{3^{9 / 2}}{2^{6}} .
$$

The proof is straightforward again and combines the corollaries in the proposition with the asymptotic formulas for $\Phi(n)$ and $I(n)$. In fact, the constant $C$ is the base of exponent in the symmetric case of the box formula (2) for $|\operatorname{PP}(n, n, n)|$, see $[70$, A008793].

From here, for $n=3 k$ and $\widehat{w}_{0} \in S_{n}$ as above, we have:

$$
\frac{\log _{2} \Upsilon_{\widehat{w}_{0}}}{n^{2}} \rightarrow \frac{2}{9} \log _{2} C \approx 0.251629 \text { as } \quad n=3 k \rightarrow \infty
$$

This is a mild improvement over Stanley's lower bound (*). ${ }^{(8)}$

Finally, for comparison, we obtain similar asymptotics for three more families of stable permutations, i.e. permutations whose diagrams have stable shape (cf. [53]).

${ }^{(8)}$ In [55] we improve this lower bound to about 0.293 and prove that this is maximal for principal Schubert evaluations of layered permutations. 
Proposition 6.5 (stable permutations). Let $v(a)=2413 \otimes 1^{a} \in S_{4 a}$ as in Corollary 5.11, $s(a)=351624 \otimes 1^{a} \in S_{6 a}$ as in Corollary 1.5, and $t(a) \in S_{8 a-2}$ as in Corollary 5.17. Then we have: $\log \Upsilon_{v(a)}=\Theta\left(a^{2}\right), \log \Upsilon_{s(a)}=\Theta\left(a^{2}\right)$, and $\log \Upsilon_{t(a)}=\Theta\left(a^{2}\right)$.

We omit the proof which is again a straightforward calculation. To compare this with Stanley's bound, take the following example:

$$
\frac{\log _{2} \Upsilon_{s(a)}}{n^{2}} \rightarrow\left(\frac{1}{4} \log _{2} 3+\frac{25}{72} \log _{2} 5-\frac{10}{9}\right) \approx 0.091354 \text { as } n=6 a \rightarrow \infty .
$$

This suggests that perhaps every family $\left\{w \in S_{n}\right\}$ of stable permutations satisfies $\Upsilon_{w}=\exp \Theta\left(n^{2}\right)$. On the other hand, as suggested by the exact computations in [48, 73 ], it is likely that that the maximum of $\Upsilon_{w}$ is achieved on a smaller class of stable Richardson permutations.

\section{LOZENGE TILINGS WITH MULTIVARIATE WEIGHTS}

In this section we study lozenge tilings of regions in the triangular grid. On a technical level, we show how the multivariate sums $G_{\lambda / \mu}(\mathbf{x} \mid \mathbf{y})$ appear in the context of lozenge tilings.

7.1. Combinatorics of LOZEnge Tilings. Let us show how excited diagrams can be interpreted as lozenge tilings of certain shapes (plane partitions) with multivariate local weights. As a consequence, the multivariate sum $G_{\lambda / \mu}(\mathbf{x} \mid \mathbf{y})$ of excited diagrams is a partition function of such lozenge tilings, and by Lemma 3.4 and the definition of factorial Schur functions it can be computed as a determinant.
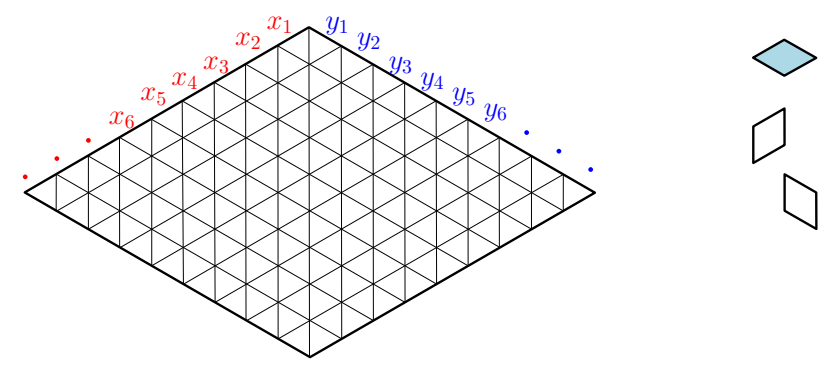

Figure 9. Triangular grid in the plane with axes $\mathbf{x}$ and $\mathbf{y}$. The grid is tiled with three types of lozenges. Horizontal lozenges at position $(i, j)$ have a weight $x_{i}-y_{j}$ (see Figure 10).

Consider the triangular grid in the plane where we identify two of the axes as $x$ and $y$, see Figure 9. Adjacent triangles can be paired into lozenges, which can tile certain prescribed regions in the plane. The lozenges whose long axis is horizontal, are called horizontal lozenges, and are colored in blue in the picture. Each of these lozenges is assigned a local weight, depending on its position with respect to the $x$ and $y$ axes. More precisely, the weight of the lozenge at position $(i, j)$, is defined to be $\left(x_{i}-y_{j}\right)$. Let $\Gamma$ be a region in the plane, and let $T$ be a tiling of $\Gamma$ (no holes, no overlaps). Let $\mathbf{h l}(T)$ denote the set of horizontal lozenges $(\diamond)$ of $T$, and let

$$
\operatorname{wt}(T):=\prod_{(i, j) \in \mathbf{h l}(T)}\left(x_{i}-y_{j}\right)
$$

be the weight of the tiling $T$.

For a partition $\mu$ and an integer $d$, consider plane partitions of base $\mu$ and height at most $d$, these correspond to the set of tilings $\Omega_{\mu, d}$ of the plane in the region whose 
lower side is given by $\mu$, and the rest is bounded by the top 4 sides of a hexagon of vertical side length $d$. Given a skew partition $\lambda / \mu$, let $\Omega_{\mu}(\lambda)$ be the set of lozenge tilings $T \in \Omega_{\mu, d}, d=\max \left\{f_{1}^{(\lambda / \mu)}-1, f_{\ell(\mu)}^{(\lambda / \mu)}-\ell(\mu)\right\}$, such that on each vertical diagonal $i-j=k$ there are no horizontal lozenges in $T$ with coordinates $(i, j)$ for $j>\lambda_{i}$. The hook weight of $T$ at position $(i, j)$ is obtained from $\operatorname{wt}(T)$ by evaluating $x_{i}=\left(\lambda_{i}-i+1\right)$ and $y_{j}=\left(-\lambda_{j}^{\prime}+j\right)$ :

$$
\mathrm{wt}_{\lambda}(T):=\prod_{(i, j) \in \mathbf{h} \mathbf{l}(T)}\left(\lambda_{i}-i+\lambda_{j}^{\prime}-j+1\right) .
$$

We define the following map between excited diagrams and lozenge tilings of base $\mu$. Let $D \in \mathcal{E}(\lambda / \mu)$, then define $\tau(D):=T$ to be the tiling $T$ with base $\mu$, such that if box $(i, j) \in D$, then $T$ has a horizontal lozenge in position $(i, j)$ in the coordinates defined above. See Figure 10 for an example of $\tau$.

EXAMPLE 7.1. There are five lozenge tilings in $\Omega_{21}(332)$ corresponding to excited diagrams from Example 2.2 :

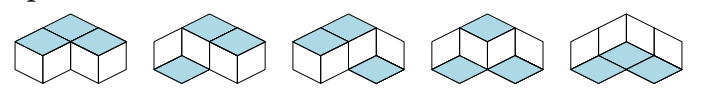

THEOREM 7.2. The map $\tau$ is a bijection between excited diagrams $\mathcal{E}(\lambda / \mu)$ and lozenge tilings $\Omega_{\mu}(\lambda)$.

Proof. We first interpret the excited diagram $D$ as a plane partition $P$ of shape $\mu$ (and nonpositive entries) under $P_{i, j}=-r_{i, j}+i$, alternatively a skew $\operatorname{RPP} P_{i, j}^{\prime}=r_{i, j}-i$, where $r_{i, j}$ is the row number of the final position of box $(i, j)$ of $\mu$ after it has been moved under the excited moves from $\mu$ to $D$. Next, $P$ corresponds to a lozenge tiling in the obvious way, where we set level 0 to be the top $z$-plane and the horizontal lozenges are moved down to the heights given by $P_{i, j}$. The condition $D \subset[\lambda]$ is equivalent to the condition that the boxes on diagonal $i-j=k$ cannot move beyond the intersection of this diagonal and $\lambda$, so that the coordinates of a horizontal lozenge $(i, j)$ must satisfy $j \leqslant \lambda_{i}$. Finally, note that excited moves correspond to flips on lozenge tilings, see e.g. [77]. Since the starting excited diagram $D=[\mu]$ corresponds to top-adjusted horizontal lozenges whose complement can be tiled by non-horizontal lozenges, the same holds for all $D \in \mathcal{E}(\lambda / \mu)$. This implies that $\tau$ is the desired bijection.

From the map $\tau$, adding the corresponding weight of the horizontal lozenges, we obtain the following result.

COROLlary 7.3. For a skew shape $\lambda / \mu$, we have

$$
G_{\lambda / \mu}(\mathbf{x} \mid \mathbf{y})=\sum_{T \in \Omega_{\mu}(\lambda)} \prod_{(i, j) \in \mathbf{h} \mathbf{l}(T)}\left(x_{i}-y_{j}\right) .
$$

As in the introduction, denote by $\mathrm{H}(a, b, c)$ the $\langle a \times b \times c \times a \times b \times c\rangle$ hexagon with base $a \times b$ and height $c$. Denote by $\mathcal{T}_{a b c}$ the set of lozenge tilings of $\mathrm{H}(a, b, c)$ weighted as in (45).

Corollary 7.4. For all $a, b, c, d, e \in \mathbb{N}$, we have

$$
\begin{aligned}
\sum_{T \in \mathcal{T}_{a b c}} \prod_{(i, j) \in \mathbf{h} \mathbf{l}(T)}(k-i-j) & \\
= & \frac{\Phi(a+b+c+d+e) \Phi(c+d+e) \Phi(a+b+c) \Phi(a) \Phi(b) \Phi(c)}{\Phi(a+c+d+e) \Phi(b+c+d+e) \Phi(a+b) \Phi(b+c) \Phi(a+c)},
\end{aligned}
$$

where $k=a+b+2 c+d+e+1$. 
First proof. By Corollary 7.3 and 3.14 we have that

$$
\sum_{T \in \mathcal{T}_{a b c}} \prod_{(i, j) \in \mathbf{h} \mathbf{l}(T)}\left(x_{i}-y_{j}\right)=\sum_{S_{\in \mathcal{E}}{ }_{\left((b+c)^{a+c} / 0^{c} b^{a}\right.}} \prod_{(i, j) \in S}\left(x_{i}-y_{j}\right) .
$$

Next we evaluate $x_{i}=k-i$ and $y_{j}=j$ to obtain the hook weight $(k-i-j)$ for each horizontal lozenge at position $(i, j)$. Note that this hook weight is constant when $(i+j)$ is constant. Thus, each NE-excited diagram on the RHS above has the same contribution to the sum. Therefore, the product in the RHS is given by:

$$
\prod_{(i, j) \in S}(k-i-j)=\prod_{(i, j) \in(b+c)^{a+c} / 0^{c} b^{a}}(k-i-j)=\frac{\Phi(a+b+c+d+e) \Phi(c+d+e)}{\Phi(a+c+d+e) \Phi(b+c+d+e)} .
$$

Lastly, the number of excited diagrams is given by (2).

Second proof. Alternatively, by Corollary 7.3 and Lemma 4.6,

$$
\sum_{T \in \mathcal{T}_{a b c}} \prod_{(i, j) \in \mathbf{h} \mathbf{l}(T)}(k-i-j)=\sum_{D \in \mathcal{E}(\lambda / \mu)} \prod_{(i, j) \in D} h_{\lambda}(i, j),
$$

where $\lambda / \mu=\Lambda(a, b, c, d, e, 0)$. By (NHLF), the RHS is equal to

$$
\frac{1}{n !} f^{\Lambda(a, b, c, d, e, 0)} \prod_{(i, j) \in \lambda} h_{\lambda}(i, j) .
$$

The result then follows form the product formula for $f^{\Lambda(a, b, c, d, e, 0)}$ from Corollary 1.1 and by taking the product of the hooks in $\lambda$.

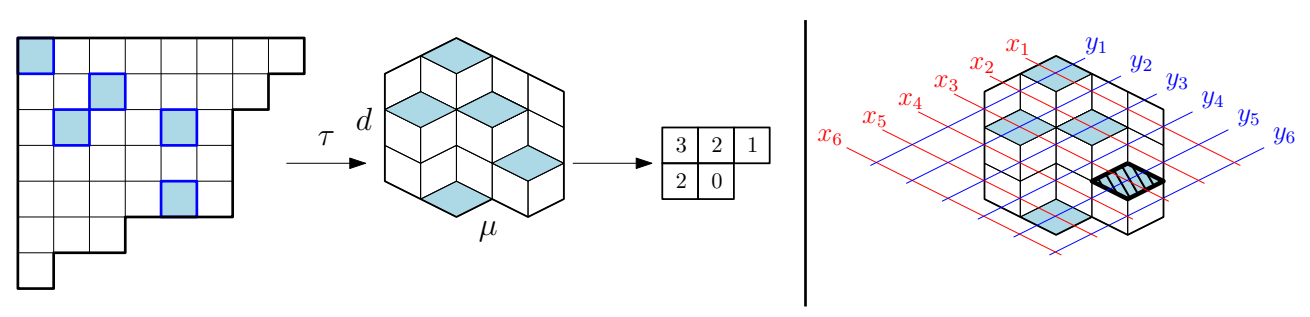

FIGURE 10. Left: the correspondence between excited diagrams with inner partition $\mu=32$, lozenge tilings with base $\mu$, and solid partitions. Right: the $x$ and $y$ coordinates giving local weights to horizontal lozenges. The highlighted horizontal lozenge has weight $\left(x_{3}-y_{5}\right)$.

7.2. Determinantal formulas for Weighted lozenge tilings. Next, we give determinantal formulas for certain multivariate sums of lozenge tilings in $\Omega_{\mu}(\lambda)$ and in $\Omega_{\mu, d}$. Recall that $\Delta(\mathbf{x})$ denotes the Vandermonde determinant.

TheOREM 7.5. Let $\lambda / \mu$ be a skew shape, and $d$ a sufficiently large positive integer, so that $\lambda / \mu \subset d \times\left(d+\mu_{1}\right)$. Let $d^{\prime}=d+\ell(\mu)$ and $n:=d^{\prime}+d+\mu_{1}$. Then:

$$
\begin{aligned}
& \sum_{T \in \Omega_{\mu}(\lambda)} \operatorname{wt}(T) \\
& \quad=\operatorname{det}\left[\left(x_{i}-y_{1}\right) \cdots\left(x_{i}-y_{\mu_{j}+L_{j}-j-1}\right)\left(x_{i}-x_{d^{\prime}}\right) \cdots\left(x_{i}-x_{L_{j}}\right)\right]_{i, j=1}^{n} \Delta(\mathbf{x})^{-1},
\end{aligned}
$$

where the sum is over lozenge tilings $T$ with base $\mu$ and height $d$, and

$$
L_{j}:=\min \left\{k: \lambda_{k}-k+1 \leqslant \mu_{j}-j\right\} .
$$


Proof. We use Corollary 7.3 to rewrite the LHS above as the sum $G_{\lambda / \mu}(\mathbf{x} \mid \mathbf{y})$ over excited diagrams. We then use Lemma 3.4 to write this sum as an evaluation of the factorial Schur function $s_{\mu}^{\left(d^{\prime}\right)}\left(\mathbf{x} \mid \mathbf{z}^{(\lambda)}\right)$ with $z_{\lambda_{i}+\left(d^{\prime}+1-i\right)}=x_{i}$ and $z_{d^{\prime}+j-\lambda_{j}^{\prime}}=y_{j}$. Next, we evaluate this factorial Schur function via (5) as a determinant of terms $\left(x_{i}-z_{1}\right) \cdots\left(x_{i}-\right.$ $\left.z_{\mu_{j}+d^{\prime}-j}\right)$. We note that $\left\{z_{1}, \ldots, z_{\mu_{j}+d^{\prime}-j}\right\}=\left\{y_{1}, \ldots, y_{\mu_{j}+L-j-1}, x_{d^{\prime}}, \ldots, x_{L}\right\}$, where $L$ gives the smallest index of $z$ which is at most $\mu_{j}+d^{\prime}-j$ and evaluates to $x$. In other words, we must have $\lambda_{L}+\left(d^{\prime}+1-L\right) \leqslant \mu_{j}+d^{\prime}-j$.

THEOREM 7.6. Consider lozenge tilings with base $\mu$ and height $d$ where $\ell:=\ell(\mu)$. Then we have:

$$
\sum_{T \in \Omega_{\mu, d}} \operatorname{wt}(T)=\operatorname{det}\left[A_{i, j}(\mu, d)\right]_{i, j=1}^{d+\ell},
$$

where

$$
\begin{aligned}
& A_{i, j}(\mu, d) \\
& = \begin{cases}\left(x_{i}-y_{1}\right) \cdots\left(x_{i}-y_{d+\ell-j}\right)\left(x_{i}-x_{i+1}\right)^{-1} \cdots\left(x_{i}-x_{d+\ell}\right)^{-1} & \text { if } j>\ell, \\
\left(x_{i}-y_{1}\right) \cdots\left(x_{i}-y_{\mu_{j}+d}\right)\left(x_{i}-x_{i+1}\right)^{-1} \cdots\left(x_{i}-x_{d+j}\right)^{-1} & \text { if } i-d<j \leqslant \ell, \\
0 & \text { if } j \leqslant i-d .\end{cases}
\end{aligned}
$$

Proof. In Theorem 7.5 we set $\lambda=\left(\mu_{1}+d\right)^{d}(\mu+d)$, where $\mu+d$ means adding $d$ to each part of the partition $\mu$. In other words, $\lambda$ has the same border as $\mu$, but endpoints shifted by $d$ on both axes. By the bijection $\tau$ from Theorem 7.2 , it follows that $\Omega_{\mu}(\lambda)$ correspond to $\mathcal{E}(\lambda / \mu)$, where the height of the lozenges is determined by how far along the diagonals the excited boxes move. By construction of $\lambda$, each diagonal has length $d$ between $\mu$ and the border, so $\Omega_{\mu}(\lambda)=\Omega_{\mu, d}$.

We apply Theorem 7.5 with the given $\lambda / \mu$ and $d$. We now plug in the value for $\lambda$ in terms of $\mu$ : $\lambda_{k+d}=\mu_{k}+d$ for $k \leqslant \ell(\mu)$ and $\lambda_{k}=d+\mu_{1}$ for $k \leqslant d$. If $k \leqslant d$, then for all $1 \leqslant j \leqslant \ell(\mu)$ we have $\lambda_{k}+1-k=d+\mu_{1}+1-k>\mu_{1}-1 \geqslant \mu_{j}-j$. If $k>d$, then $\lambda_{k}+1-k=\mu_{k-d}+1+d-k=\mu_{i^{\prime}}+1-i^{\prime}$, where $i^{\prime}=k-d$. Then we see that for $j \leqslant \ell(\mu)$ we have $L_{j}=\min \left\{k: \lambda_{k}+1-k \leqslant \mu_{j}-j\right\}=d+\min \left\{i^{\prime}: \mu_{i^{\prime}}+1-i^{\prime} \leqslant \mu_{j}-j\right\}=d+j+1$. For $j>\ell(\mu)$, we must have $L_{j}=d+\ell(\mu)+1$ and there are no $\left(x_{i}-x_{d^{\prime}}\right) \cdots$ terms. Finally, we observe that $\Delta(\mathbf{x})=\prod_{i}\left(x_{i}-x_{i+1}\right) \cdots\left(x_{i}-x_{d^{\prime}}\right)$ and divide each entry on line $i$ by the corresponding product $\left(x_{i}-x_{i+1}\right) \cdots$.

COROLlary 7.7. Consider lozenge tilings with base $\mu$, such that $\ell(\mu)=\ell$ and height $d$, such that horizontal lozenges at position $(i, j)$ have weight $x_{i}$. Then we have the following formula for the partition function:

$$
\sum_{T \in \Omega_{\mu, d}} \prod_{(i, j) \in \mathbf{h l}(T)} x_{i}=\operatorname{det}\left[B_{i, j}\right]_{i, j=1}^{\ell+d},
$$

where

$$
B_{i, j}= \begin{cases}x_{i}^{d+\ell-j}\left(x_{i}-x_{i+1}\right)^{-1} \cdots\left(x_{i}-x_{d+\ell}\right)^{-1} & \text { if } j>\ell(\mu), \\ x_{i}^{\mu_{j}+d}\left(x_{i}-x_{i+1}\right)^{-1} \cdots\left(x_{i}-x_{d+j}\right)^{-1} & \text { if } i-d<j \leqslant \ell, \\ 0 & \text { if } j \leqslant i-d .\end{cases}
$$

Proof. We the apply Theorem 7.6 with $y_{j}=0$ and $d^{\prime}=d+\ell$ an obtain the result.

As a byproduct of our calculations we obtain the following determinant formula given in [33, Thm. 6.1] with $\alpha=\beta=0$. To state this formula we use the standard notation of the $q$-Pochhammer symbol $(a ; q)_{m}:=(1-a)(1-a q) \cdots\left(1-a q^{m-1}\right)$. 
Corollary 7.8 ([33]). Consider the set $P P_{\mu}(d)$ of plane partitions of base $\mu$ and entries less than or equal to $d$. Then their volume generating function is given by the following determinantal formula

$$
\sum_{P \in P P_{\mu}(d)} q^{|P|}=q^{N(\mu)} \operatorname{det}\left[C_{i, j}\right]_{i, j=1}^{\ell+d},
$$

where

$$
\begin{gathered}
N(\mu)=\sum_{r=1}^{\ell(\mu)} r \mu_{r}, \\
C_{i, j}= \begin{cases}(-1)^{d+\ell-i} q^{\alpha}(q ; q)_{d+\ell-i}^{-1} & \text { if } j>\ell(\mu), \\
(-1)^{d+j-i} q^{\beta}(q ; q)_{d+j-i}^{-1} & \text { if } i-d<j \leqslant \ell, \\
0 & \text { if } j \leqslant i-d,\end{cases}
\end{gathered}
$$

and

$$
\begin{aligned}
& \alpha=(d-i)(d+\ell-j)-(d-i+\ell)(d-i-\ell-1) / 2, \\
& \beta=(d-i)\left(\mu_{j}+d\right)-(d+j-i)(d-i-j-1) / 2 .
\end{aligned}
$$

Proof. Let $P \in P P_{\mu}(d)$. As explained in the proof of Theorem 7.2, it corresponds to a lozenge tiling $T \in \Omega_{\mu, d}$, where the heights of the horizontal lozenges are equal to the corresponding entries in $P$. Suppose that $P_{r, c}=m$, then the corresponding horizontal lozenge has coordinates given by $(i, j)=(r+d-m, c+d-m)$, i.e. shifted by $(d-m)$ along the diagonal. Let $x_{i}=q^{d-i}$. Then:

$$
\prod_{(i, j) \in \mathbf{h} \mathbf{l}(T)} x_{i}=\prod_{(i, j) \in \mathbf{h} \mathbf{l}(T)} q^{d-i}=\prod_{(r, c) \in \mu} q^{d-r-d+P_{r, c}}=\prod_{(r, c) \in \mu} q^{-r} q^{P_{r, c}}=q^{|P|-N(\mu)} .
$$

Therefore, substituting $x_{i} \leftarrow q^{d-i}$ in Corollary 7.7 gives the desired generating function

$$
\sum_{P \in P P_{\mu}(d)} q^{|P|}=q^{N(\mu)} \sum_{T \in \Omega_{\mu, d}} \prod_{(i, j) \in \mathbf{h l}(T)} q^{d-i} .
$$

Thus, the entries in the corresponding determinant are given by

$$
\begin{aligned}
A_{i, j}(\mu, d) & \left.\right|_{x_{i}=q^{d-i}, y_{j}=0} \\
& = \begin{cases}q^{(d-i)(d+\ell-j)}\left(q^{d-i}-q^{d-i-1}\right)^{-1} \cdots\left(q^{d-i}-q^{-\ell}\right)^{-1} & \text { if } j>\ell, \\
q^{(d-i)\left(\mu_{j}+d\right)}\left(q^{d-i}-q^{d-i-1}\right)^{-1} \cdots\left(q^{d-i}-q^{-j}\right)^{-1} & \text { if } i-d<j \leqslant \ell, \\
0 & \text { if } j \leqslant i-d .\end{cases}
\end{aligned}
$$

We can simplify the entries as

$$
\frac{q^{(d-i)(d+\ell-j)}}{\left(q^{d-i}-q^{d-i-1}\right) \cdots\left(q^{d-i}-q^{-\ell}\right)}=\frac{(-1)^{d+\ell-i} q^{(d-i)(d+\ell-j)-(d-i+\ell)(d-i-\ell-1) / 2}}{(q ; q)_{d+\ell-i}}
$$

and

$$
\frac{q^{(d-i)\left(\mu_{j}+d\right)}}{\left(q^{d-i}-q^{d-i-1}\right) \cdots\left(q^{d-i}-q^{-j}\right)}=\frac{(-1)^{d+j-i} q^{(d-i)\left(\mu_{j}+d\right)-(d+j-i)(d-i-j-1) / 2}}{(q ; q)_{d+j-i}},
$$

which imply the result.

\section{Probabilistic applications}

Here we present the main application of results in the previous section: product formulas for the probabilities of two special paths in lozenge tilings of a hexagon with hook weights of combinatorial significance. 
8.1. Path Probabilities. From here on, we assume that $x_{i}, y_{j} \in \mathbb{R}$, that $\left(x_{i}-y_{j}\right) \geqslant 0$ for all $1 \leqslant i \leqslant a$ and $1 \leqslant j \leqslant b$ and that $x_{i} \neq x_{j}$ for $i \neq j$. Recall that $\left|\mathcal{T}_{a b c}\right|$ is given by the MacMahon box formula (2). The uniform distribution on $\mathcal{T}_{a b c}$ is the special case of (45) with $x_{i}=1$ and $y_{j}=0$, for all $i, j$ as above.

In the hexagon $\mathrm{H}(a, b, c)$, consider a path $\mathrm{p}=\mathrm{p}\left(d_{0}, d_{1}, \ldots, d_{a+b}\right)$ passing through non-horizontal lozenges and consisting of unit length segments with endpoints $\left(i, d_{i}+\right.$ $1 / 2$ ). Here $i$ is indexing the vertical line, starting with $i=0$ at the leftmost end of the hexagon, and $d_{i}+1 / 2$ is the Euclidean distance measured along that vertical line to its intersection with the top axes $x>0$ or $y>0$, depending on which part the vertical line intersects them. Note that we necessarily have $d_{0}=d_{a+b},\left|d_{i}-d_{i+1}\right| \leqslant 1$, $d_{i} \leqslant d_{i+1}$ if $i \leqslant a$, and $d_{i} \geqslant d_{i+1}$ if $i>a$. Denote by $\operatorname{Prob}(\mathrm{p})$ the probability that a random weighted lozenge tiling in $\mathcal{T}_{a b c}$ contains the path $\mathrm{p}$. In addition, given a partition $\mu \subset b^{a}$, denote by $\mu^{*}$ the complement of $\mu$ in $b^{a}$.

EXAMPLE 8.1. Figure 11(a) shows an example of a tiling of a hexagon with $a=2$, $b=3$ and height $c=4$, with a path $\mathrm{p}=\mathrm{p}(2,3,3,3,3,2)$ dividing the boxed plane partition into tilings with base $\mu=31$ given by its diagonals $(0,1,1,1,1,0)$, and $\mu^{*}=20$.

Theorem 8.2. Let $x_{i}, y_{j} \in \mathbb{R}, 1 \leqslant i \leqslant a, 1 \leqslant j \leqslant b$, s.t. $\min \left\{x_{i}\right\} \geqslant \max \left\{y_{j}\right\}$. Consider the distribution on lozenge tilings $T$ of the hexagon $\mathrm{H}(a, b, c)$, weighted by the product $w(T)$ of $\left(x_{i}-y_{j}\right)$ over all horizontal lozenges. The partition function is then given by

$$
Z(a, b, c):=\sum_{T \in \mathcal{T}_{a b c}} \operatorname{wt}(T)=\operatorname{det}\left[M_{i, j}\right]_{i, j=1}^{a+c}
$$

where

$M_{i, j}= \begin{cases}\left(x_{i}-y_{1}\right) \cdots\left(x_{i}-y_{c+a-j}\right)\left(x_{i}-x_{i+1}\right)^{-1} \cdots\left(x_{i}-x_{c+a}\right)^{-1} & \text { if } j>a, \\ \left(x_{i}-y_{1}\right) \cdots\left(x_{i}-y_{b+c}\right)\left(x_{i}-x_{i+1}\right)^{-1} \cdots\left(x_{i}-x_{c+j}\right)^{-1} & \text { if } i-c<j \leqslant a, \\ 0, & \text { if } j \leqslant i-c .\end{cases}$

Moreover, the probability of a path $\mathrm{p}=\mathrm{p}\left(d_{0}, d_{1}, \ldots, d_{a+b}\right)$ in a random lozenge tiling $T \in \mathcal{T}_{\text {abc }}$ weighted $w(T)$, is given by

$$
\operatorname{Prob}(\mathrm{p})=\frac{\operatorname{det}\left[A_{i, j}\left(\mu, d_{1}\right)\right] \operatorname{det}\left[A_{i, j}^{*}\left(\mu^{*}, c-d_{1}-1\right)\right]}{Z(a, b, c)},
$$

where the partition $\mu$ with $\ell(\mu)=a$ is given by its diagonals $\left(0, d_{2}-d_{1}, d_{3}-d_{1}, \ldots\right)$, and $\mu^{*}$ is the complement of $\mu$ in $b^{a}$. Here the matrix $A$ is defined as in Theorem 7.6, while the matrix $A^{*}$ is defined similarly, after the substitution $x_{i} \leftarrow x_{a+c+1-i}, y_{j} \leftarrow$ $y_{b+c+1-j}$.

Proof. The formula for the partition function follows from a direct application of Theorem 7.6 with base $b^{a}$ and height $d=c$. For the probability, notice that the path $\mathrm{p}$ divides the boxed plane partition via a horizontal section along the path, and the partitions $\mu$ and $\mu^{*}$ are the corresponding bases outlined by the path. For the tiling with base $\mu^{*}$ we observe that it corresponds to a change of the coordinates with origin at the bottom corner of the hexagon, which corresponds to flipping the $x$ and $y$ coordinates in the opposite order.

8.2. First example. Denote by $\operatorname{Prob}_{\lambda}(\mathrm{p})$ the probability $\operatorname{Prob}(\mathrm{p})$ in the special case of hook weight $\mathrm{wt}_{\lambda}(\cdot)$ defined in (46). 


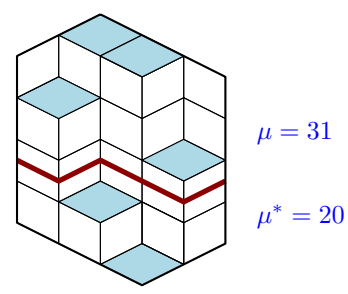

(a)

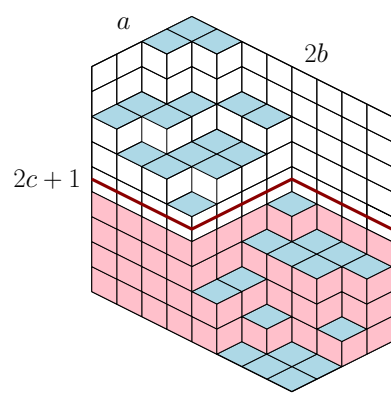

(b)

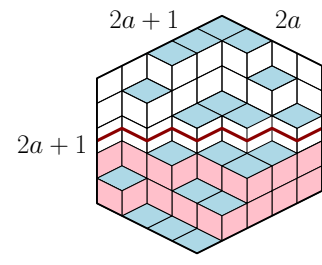

(c)

FiguRE 11. (a) example of a tiling of the region in Example 8.1 with the path $\mathrm{p}=\mathrm{p}(2,3,3,3,3,2)$, (b) example of a tiling of the region in Corollary 8.3 for $a=b=c=4$, (c) example of the tiling of region in Corollary 8.4 for $a=2$.

COROLlary 8.3. Fix $a, b, c \in \mathbb{N}$, partition $\lambda=(2 b+2 c+1)^{a+2 c+1}$, and denote $k=$ $a+2 b+4 c+3$. Let $\mathrm{wt}_{\lambda}(T)$ be the corresponding hook weight of a lozenge tiling $T$ of the hexagon $\mathrm{H}(a, 2 a, 2 a+1)$. Finally, if $a \leqslant b$ (and analogously if $a>b)$, let $\mathrm{p}$ be the following path in $\mathrm{H}(a, 2 b, 2 c+1)$ of length $(a+2 b)$ :

$$
\mathrm{p}:=\mathrm{p}\left(c, c+1, \ldots,(c+a)^{b-a}, c+a-1, \ldots, c^{b}\right) .
$$

Then:

$$
\operatorname{Prob}_{\lambda}(\mathrm{p})=\frac{Q(a, b, c, 0,0) \cdot Q(a, b, c, c+1,2 c+1)}{Q(a, 2 b, 2 c+1,0,0)},
$$

where $Q(a, b, c, d, e)$ is the RHS of (47).

The choice of weights here is made to correspond to counting of the SYT in the previous section. See Figure 11(b) for an illustration.

Proof. The path $\mathrm{p}$ partitions the rectangle $[a \times 2 b]$ into $\mu=\mu^{*}=b^{a}$. By the proof of Theorem 8.2

$$
\operatorname{Prob}_{\lambda}(\mathrm{p})=\frac{N\left(b^{a}\right) \cdot N^{*}\left(b^{a}\right)}{Z(a, 2 b, 2 c+1)}
$$

where

$$
N\left(b^{a}\right):=\sum_{T \in \mathcal{T}_{a b c}} \mathrm{wt}(T),
$$

and $N^{*}\left(b^{a}\right)$ equals $N\left(b^{a}\right)$ after the substitution $x_{i} \leftarrow x_{a+2 c+2-i}, y_{j} \leftarrow y_{2 b+2 c+2-j}$.

Next, we evaluate $x_{i}=k-i$ and $y_{j}=j$ in (48) to obtain the hook weight of the tiling and thus get $\operatorname{Prob}_{\lambda}(\mathrm{p})$. By Corollary 7.4 for the hexagon $\mathrm{H}(a, 2 b, 2 c+1)$, we have:

$$
\left.Z(a, 2 b, 2 c+1)\right|_{\substack{x_{i}=k-i \\ y_{j}=j}}=Q(a, 2 b, 2 c+1,0,0) .
$$

By Corollary 7.4 for the hexagon $\mathrm{H}(a, b, c)$, we have:

$$
\left.N\left(b^{a}\right)\right|_{\substack{x_{i}=k-i \\ y_{j}=j}}=Q(a, b, c, c+1,2 c+1),
$$

and

$$
\left.N^{*}\left(b^{a}\right)\right|_{\substack{x_{i}=k-i \\ y_{j}=j}}=Q(a, b, c, 0,0) .
$$

Together, these imply the desired expression for $\operatorname{Prob}_{\lambda}(\mathrm{p})$. 
In the notation of the proposition, let $a=b=c$. Then $\mathrm{P}_{w}(a):=\operatorname{Prob}_{\lambda}(\mathrm{p})$ is exactly the probability that the random hook weighted lozenge tiling of $\mathrm{H}(a, 2 a, 2 a+1)$ has two frozen $\langle a \times a\rangle$ rhombi as in Figure 11(b), where the weights are chosen to correspond to SYT counting (cf. figures 2 and 17). Of course, in the uniform case the corresponding probability $\mathrm{P}_{u}(a)$ is a little easier to compute:

$$
\mathrm{P}_{u}(a)=\frac{|\mathrm{PP}(a, a, a)|^{2}}{|\operatorname{PP}(a, 2 a, 2 a+1)|},
$$

see the MacMahon box formula (2). A direct calculation shows that

$$
\log \mathrm{P}_{w}(a)=\alpha a^{2}+O(a \log a), \quad \log \mathrm{P}_{u}(a)=\beta a^{2}+O(a \log a) \quad \text { and } \alpha<\beta<0 .
$$

Since there are $\left(\begin{array}{c}3 a \\ a\end{array}\right)=\exp O(a)$ possible paths with the same endpoints as $\mathrm{p}$, this shows that $\mathrm{p}$ is exponentially unlikely in both cases, and even less likely in the hook weighted lozenge tiling.

8.3. SECOND EXAMPLE. Our next example uses the number of excited diagrams of thick ribbons $\delta_{2 a+1} / \delta_{a}$, studied extensively in [53, 55].

Corollary 8.4. Fix $a \in \mathbb{N}$, partition $\lambda=(4 a+1)^{4 a+2}$, and let $\mathrm{wt}_{\lambda}(T)$ be the corresponding hook weight of a lozenge tiling $T$ of the hexagon $\mathrm{H}(2 a+1,2 a, 2 a+1)$. Finally, let $\mathrm{q}$ be the zigzag path in $\mathrm{H}(2 a+1,2 a, 2 a+1)$ of length $(4 a+1)$ :

$$
\mathrm{q}:=(a, a, a+1, a+1, a+2, a+2, \ldots, 2 a, 2 a, \ldots, a, a) .
$$

Then:

$$
\frac{C(a)}{|\mathrm{PP}(2 a+1,2 a, 2 a+1)|} \leqslant \operatorname{Prob}_{\lambda}(\mathbf{q}) \leqslant \frac{C(a) \cdot\left|\mathrm{RPP}_{\delta_{2 a+1}}(a)\right|^{2}}{|\mathrm{PP}(2 a+1,2 a, 2 a+1)|},
$$

where $\left|\operatorname{RPP}_{\delta_{2 a+1}}(a)\right|$ is given by $(38)$, and

$$
C(a)=\frac{\Phi(8 a+3) \Phi(4 a+2)(4 a) !^{2 a+1}}{\Phi(6 a+2)^{2}(6 a+2) !^{2 a+1}(2 a) !} .
$$

Proof. The proof follows along the same lines as the proof of Corollary 8.3 above. The path q partitions the rectangle $[(2 a+1) \times 2 a]$ into shapes $\mu$ and $\mu^{*}$, where $\mu=\mu^{*}=\delta_{2 a+1}$. By the proof of Theorem 8.2

$$
\operatorname{Prob}_{\lambda}(\mathbf{q})=\frac{N\left(\delta_{2 a+1}\right) N^{*}\left(\delta_{2 a+1}\right)}{Z(2 a+1,2 a, 2 a+1)},
$$

where

$$
N\left(\delta_{2 a+1}\right):=\sum_{T \in \Omega_{\delta_{2 a+1}, a}} \prod_{(i, j) \in \mathbf{h l}(T)} \mathrm{wt}(T),
$$

and $N^{*}\left(\delta_{2 a+1}\right)$ is equal to $N\left(\delta_{2 a+1}\right)$ after the substitution $x_{i} \leftarrow x_{4 a-i+3}$ and $y_{j} \leftarrow$ $y_{4 a-j+2}$. Next, we evaluate $x_{i}$ and $y_{j}$ as specified to obtain the hook weight of the tiling and get $\operatorname{Prob}_{\lambda}(\mathrm{q})$ (see figures 12(a) and 12(b)).

By a similar argument to the first proof of Corollary 7.4, the partition function of the denominator factors as follows:

$$
\left.Z(2 a+1,2 a, 2 a+1)\right|_{\mathrm{wt}_{(T)=\mathrm{wt}_{\lambda}(T)}}=\frac{\Phi(6 a+2) \Phi(2 a+1)}{\Phi(4 a+2) \Phi(4 a+1)}|\operatorname{PP}(2 a+1,2 a, 2 a+1)| .
$$

We have:

$$
\left.N\left(\delta_{2 a+1}\right)\right|_{\mathrm{wt}(T)=\mathrm{wt}_{\lambda}(T)}=\sum_{D \in \mathcal{E}(\pi)} \prod_{(i, j) \in D} h_{\lambda}(i, j)
$$




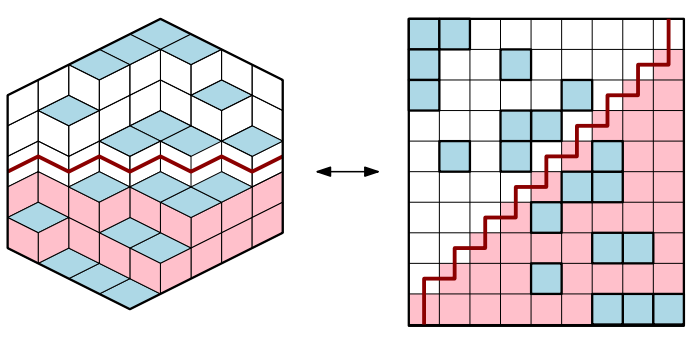

(a)

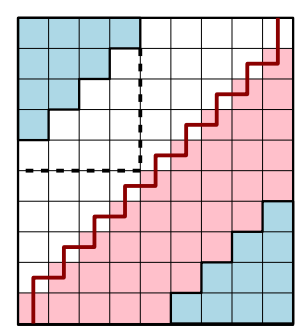

(b)

FiguRE 12. (a) excited diagram interpretation of the example of a tiling of the region in Corollary 8.4; (b) skew shapes on both sides of the path $\mathrm{q}$ and values of $x_{i}, y_{j}$ of this example.

By the proof of the bound in [53, Thm. 1.1], we have:

$$
\frac{\Phi(8 a+3)}{\Phi(6 a+3) \cdot(6 a+2) !^{2 a}} \leqslant\left. N\left(\delta_{2 a+1}\right)\right|_{\mathrm{wt}(T)=\mathrm{wt}_{\lambda}(T)} \leqslant \frac{\Phi(8 a+3) \cdot\left|\mathrm{RPP}_{\delta_{2 a+1}}(a)\right|}{\Phi(6 a+3) \cdot(6 a+2) !^{2 a}} .
$$

A similar calculation for $N^{*}\left(\delta_{2 a+1}\right)$ gives

$$
\frac{\Phi(2 a)(4 a) !^{2 a}}{\Phi(4 a)} \leqslant\left. N^{*}\left(\delta_{2 a+1}\right)\right|_{\mathrm{wt}(T)=\mathrm{wt}_{\lambda}(T)} \leqslant \frac{\Phi(2 a)(4 a) !^{2 a}}{\Phi(4 a)}\left|\operatorname{RPP}_{\delta_{2 a+1}}(a)\right| .
$$

Applying these bounds on the RHS of (50) gives the desired result.

In the notation of the proposition, the probability $\mathrm{P}_{w}(a):=\operatorname{Prob}_{\lambda}(\mathrm{q})$ is exactly the probability that the random hook weighted lozenge tiling of the hexagon $\mathrm{H}(2 a+$ $1,2 a, 2 a+1)$ has a horizontal zigzag path as in Figure 11(c), splitting the hexagon into two equal shapes.

For comparison, in the uniform case, the corresponding probability $\mathrm{P}_{u}(a)$ is given by:

$$
\mathrm{P}_{u}(a)=\frac{\left|\operatorname{RPP}_{\delta_{2 a+1}}(a)\right|^{2}}{|\mathrm{PP}(2 a+1,2 a, 2 a+1)|},
$$

so the second inequality in the corollary can be written as

$$
\mathrm{P}_{w}(a) \leqslant C(a) \mathrm{P}_{u}(a) .
$$

Now direct calculation using the bounds above gives a remarkable contrast between the asymptotics:

$$
\log \mathrm{P}_{w}(a)=\Theta\left(a^{2}\right), \quad \log \mathrm{P}_{u}(a)=\gamma a+O(\log a) \quad \text { for some } \quad \gamma<0 .{ }^{(9)}
$$

This supports the intuition that for the uniform distribution path $\mathrm{q}$ is at least as likely as any other path among the $\left(\begin{array}{c}4 a+1 \\ 2 a\end{array}\right)$ possible, while for the hook weighted distribution path $\mathrm{q}$ is extremely unlikely. In the language of limit shapes in Figure 2, this says that in the uniform case, the limit shape (the Arctic circle) touches the vertical sides in the middle, while in the hook weighted case it touches someplace higher.

EXAMPLE 8.5. In the case $a=1$, we have: $\lambda=5^{6}, C(1)=54 / 35,|\operatorname{PP}(3,2,3)|=175$ and $\left|\operatorname{RPP}_{\delta_{3}}(1)\right|=5$. The bounds for $\mathrm{P}_{w}(1)=\operatorname{Prob}(\mathrm{q})$ given by (49), are:

$$
\frac{54}{6125} \approx 0.0088 \leqslant \mathrm{P}_{w}(1) \leqslant \frac{54}{245} \approx 0.2204 \text {. }
$$

${ }^{(9)}$ While we certainly believe that $\log \mathrm{P}_{w}(a)=\tau a^{2}+o\left(a^{2}\right)$, for some $\tau<0$, the bounds in Corollary 8.4 are too weak to establish that. It would also be interesting to compute $\tau$ explicitly. 
The actual value of the probability is $\mathrm{P}_{w}(1)=246 / 4375 \approx 0.0562$. On the other hand, in the uniform case we have $\mathrm{P}_{u}(1)=1 / 7 \approx 0.1429$. Note that here we have $C(1)>1$, while asymptotically $C(a)<\exp \left(-c a^{2}\right)$, for some $c>0$.

\section{Final Remarks}

9.1. HistoricAl nOtes. The hook-length formula (HLF) plays an important role in both enumerative and algebraic combinatorics, and has a large number of proofs, extensions and generalizations. We refer to [1] for a comprehensive recent survey, and to $[54, \S 9]$ for a review of the NHLF and other formulas generalizing $f^{\lambda / \mu}=$ $|\operatorname{SYT}(\lambda / \mu)|$.

Likewise, the subject of domino and lozenge tilings is a large subject in its own right, with many determinant and product formulas (notably, for the Aztec diamond), weighted extensions, asymptotic and probabilistic results. We refer to $[6,40]$ for extensive recent discussions of both and overview of the literature. Note that even among other tiling problems, domino and lozenge tilings are special to have both determinantal formulas and height functions (see [60]).

Finally, the subject of Schubert polynomials has several enumerative formulas including Macdonald's identity (8). One of the most celebrated product formula $\left|R\left(w_{0}\right)\right|=f^{\delta_{n}}$, where $w_{0} \in S_{n}$ is the longest permutation, is due to Stanley. It is now generalized in many directions including the Fomin-Kirillov identity (Corollary 5.7). The formula (37) for Schubert polynomials of vexillary permutations appears in the literature in terms of flagged Schur functions of shape $\mu(w)$ [47, Thm. 2.6.9]. Also, the Schubert polynomial of a 321-avoiding permutation $w$ is a flagged skew Schur function of $\operatorname{shape} \operatorname{skewsh}(w)[8$, Thm. 2.2]. We refer to $[43,47]$ for detailed introductions to the area.

9.2. Bijective proofs For Product formulas. The product formulas in Corollaries 1.1-1.3 and their $q$-analogues beg for bijective or hook-walk type proofs, see [59, 20]. We should warn the reader, however, of many related product formulas which have yet to have a bijective proof. Most famous of this is the product formula for the number of alternating sign matrices (ASM), which in Kuperberg's proof comes out as an evaluation of a "hidden symmetry" of a multivariate determinant, of similar flavor to our proof [38] (see also [11, 39]).

Similarly, some years ago the second author proposed giving a combinatorial proof of the Selberg integral by proving an explicit product formula with several parameters counting linear extensions of certain posets (see [74, Ex. 3.11(b)]). The product formulas are superficially similar in flavor to the ones in Corollary 1.1 due to the structure of parameters; in fact they look even simpler. While this project is yet to be realized, this connection was used in reverse direction in an elegant paper [25].
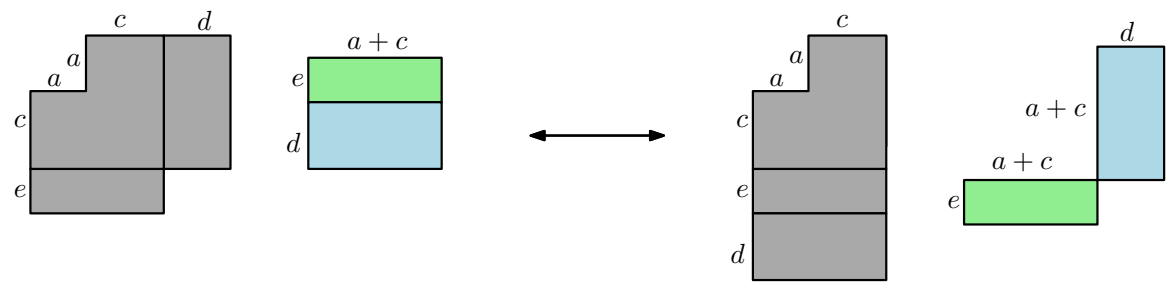

Figure 13. Potential bijection for $\pi=\Lambda(a, a, c, d, e, 0)$.

In a positive direction, we should mention that in the special case of $\pi=$ $\Lambda(a, a, c, d, e, 0)$, Corollary 1.1 implies that there is a mysterious identity for 


$$
\begin{aligned}
& f^{\pi}=|\operatorname{SYT}(\pi)|: \\
& \begin{aligned}
&|\operatorname{SYT}(\pi)| \cdot\left|\operatorname{SYT}\left((a+c)^{d+e}\right)\right|\left|\operatorname{SYT}\left((a+c)^{a+c+d+e} / a^{a}\right)\right| \\
& \times\left(\begin{array}{c}
(a+c)(d+e) \\
(a+c) e
\end{array}\right) \cdot\left|\operatorname{SYT}\left((a+c)^{e}\right)\right| \cdot\left|\operatorname{SYT}\left((a+c)^{d}\right)\right| .
\end{aligned}
\end{aligned}
$$

Since all other terms in the product do have a bijective proof of the corresponding product formulas, a bijective proof of this identity would imply a (rather involved combined) bijective proof of the product formula for $|\mathrm{SYT}(\pi)|$.

Finally, we should note that our Theorem 4.1 should be viewed as a stand-alone coincidence rather than beginning of the emerging pattern. In some sense, we are really saying that for certain families of skew shapes the determinantal formula for $f^{\lambda / \mu}$ can be further simplified to a product formula. Thus our product formulas have a natural home in Determinantal Calculus [34,35] and lozenge tilings literature (see e.g. $[40,41])$, rather than the general study of linear extensions of posets.

9.3. Kiм-Oh's тheOREm. We learned about [27] only after this paper was finished. They prove Corollary 1.1 via a product formula for Young books: pairs of SYT of shifted shape $\Lambda^{\nabla}(a, c, d, 0)$ and $\Lambda^{\nabla}(b, c, e, 0)$ with the same diagonal entries (see $\S 9.5$ for a definition of the shape $\Lambda^{\nabla}$ ). Their tools cannot be used to derive our main product formula in Theorem 4.1. This would require a version of Young books of shapes $\Lambda^{\nabla}(a, c, d, m)$. Note that the $q$-analogue in Corollary 4.3 does not follow from [25], but perhaps follows from a $q$-Selberg integral generalization of [25] given in [28].

9.4. DeWitt's theorem. The case of the shape $\Lambda(a, b, c, 0,0,1)$ in Corollary 1.2 might be the most tractable since its product formula is known to count another natural object as we explain next. DeWitt showed in her thesis [13] that in this case $f^{\lambda / \mu}$ counts, up to a power of 2 , the number of SYT of a shifted shape. Given nonnegative integers $a, b, c$ let $T(a, b)$ be the trapezoid

$$
T(a, b):=(a+b-1, a+b-3, \ldots,|b-a|+1),
$$

and let $D(a, b, c)$ be the shifted shape obtained by flipping by the diagonal $y=-x$ the shifted skew shape $\delta_{a+b+2 c} / T(a, b)$. See Figure 14 for an example of this shape.

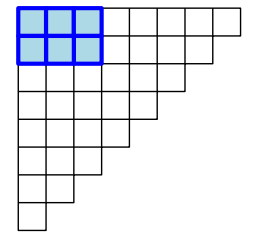

$\Lambda(2,3,2,0,0,1)$

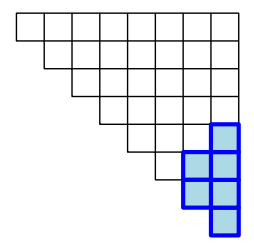

$D(2,3,2)$

Figure 14. Examples of the skew shape $\Lambda(a, b, c, 0,0,1)$ and the shifted shape $D(a, b, c)$ that have equinumerous SYT.

Theorem 9.1 (Thm. V.3 [13]). For the skew shape $\lambda / \mu=\delta_{a+b+2 c} / b^{a}$, we have:

$$
s_{\lambda / \mu}=P_{D(a, b, c)} .
$$

By taking the coefficient of $x_{1} x_{2} \ldots x_{n}$ in the equation above we obtain the following identity between the number of SYT of skew shape $\Lambda(a, b, c, 0,0,1)$ and shifted shape $D(a, b, c)$. 
Corollary 9.2 ([13, Cor. V.7]). For the skew shape $\lambda / \mu=\delta_{a+b+2 c} / b^{a}$, we have:

$$
f^{\lambda / \mu}=2^{|\lambda / \mu|-a-b-2 c+1} g^{D(a, b, c)} .
$$

Combining this identity with the hook-length formula for $g^{\nu}$ (see e.g. [67, Ex. 3.21]), we obtain a product formula for $f^{\Lambda(a, b, c, 0,0,1)}$ coinciding with that of Corollary 1.2. Similarly, by doing a stable principal specialization in (51) and using the Kawanaka product formula [23] for this specialization of Schur $P$-functions of straight shifted shapes, we obtain the formula in Corollary 4.4. This identity was obtained earlier and more generally by Krattenthaler and Schlosser, see Eq. (1.2) with $n=a+b+2 c$, $m=b$ and $r=a$ in [36].

Note that DeWitt and Ardila-Serrano [2] showed independently that the skew Schur function $s_{\delta_{m} / \mu}$ has a positive expansion in the Schur $P$-functions. From this expansion one can obtain Theorem 9.1.

It is natural to ask for a bijective proof of Corollary 9.2. Such a bijection combined with the hook-walk algorithm for shifted shapes [66] or the bijective proof of the hook-length formula for $g^{\nu}$ [15], gives an algorithm to generate SYT of skew shape $\Lambda(a, b, c, 0,0,1)$ uniformly at random. We obtain the desired bijection in the followup work [50].

9.5. Shifted SHAPES. One of the main results of this paper is to give families of skew shapes whose number of SYT is given by a product formula. A natural direction is to study the same question for shifted skew shapes.

Naruse in [57] also announced two formulas (of type $B$ and type $D$ ) for the number of standard tableaux of shifted skew shape (see $[52, \S 8]$ ), in terms of analogues of excited diagrams. ${ }^{(10)}$

The type $B$ excited diagrams are obtained from the diagram of $\mu$ by applying the following type $B$ excited moves:

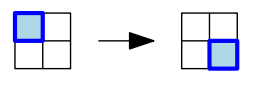

and

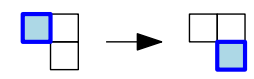

We denote the set of type $B$ excited diagrams of shifted skew shape $\lambda / \mu$ by $\mathcal{E}^{B}(\lambda / \mu)$. Following the arguments in Section 2.7 and $[54, \S 3]$, the type $B$ excited diagrams of $\lambda / \mu$ are equivalent to certain flagged tableaux of shifted shape $\mu$ and to certain non-intersecting paths (cf. [76]).

Question 9.3. Is there a determinantal or Pfaffian formula for $\left|\mathcal{E}^{B}(\lambda / \mu)\right|$ counting the corresponding flagged tableaux of shifted shape $\mu$ ?

Given a shifted shape $\lambda$, the type $B$ hook of a cell $(i, i)$ in the diagonal is the set of cells in row $i$ of $\lambda$. The hook of a cell $(i, j)$ for $i \leqslant j$ is the set of cells in row $i$ right of $(i, j)$, the cells in column $j$ below $(i, j)$, and if $(j, j)$ is one of these cells below then the hook also includes the cells in the $j$ th row of $\lambda$, thus counting $(j, j)$ twice overall. See Figure 15. The NHLF then extends verbatim for the number $g^{\lambda / \mu}$ of standard tableaux of shifted skew shape $\lambda / \mu$.

TheOREM 9.4 (Naruse [57]). Let $\lambda, \mu$ be partitions with distinct parts, such that $\mu \subset \lambda$. We have

$$
g^{\lambda / \mu}=|\lambda / \mu| ! \sum_{S \in \mathcal{E}^{B}(\lambda / \mu)} \prod_{(i, j) \in[\lambda] \backslash S} \frac{1}{h^{B}(i, j)} .
$$

Next we describe the shifted analogue of the thick reverse hook (Example 2.5).

\footnotetext{
${ }^{(10)}$ This result was recently proved and further generalized in [58].
} 
EXAMPLE 9.5 (shifted reverse hook). For the shape $R_{a, c}:=\delta_{a+c+1} / \delta_{a+1}$, the type $B$ excited diagrams correspond to symmetric plane partitions with at most $a+1$ rows and largest part at most $c$. By the Andrews-Gordon formula for symmetric plane partitions (see [71]), we have:

$$
\left|\mathcal{E}^{B}\left(R_{a, c}\right)\right|=\prod_{1 \leqslant i \leqslant j \leqslant a} \prod_{k=1}^{c} \frac{i+j+k-1}{i+j+k-2}=\frac{\Phi(2 a+c) \Phi(a)}{\Phi(2 a) \Phi(a+c)} \cdot \frac{\Xi(2 a) \beth(c)}{\beth(2 a+c)} .
$$

It is natural to study shifted analogues of our product formulas for skew shapes. For nonnegative integers $a \leqslant c, d$ and $m$, let $\lambda / \mu=\Lambda^{\nabla}(a, c, d, m)$ be the following shifted skew partition

$$
\lambda=(c+a, c+a-1, \ldots, 1)+\nu,
$$

where $\nu=(d+(a+c-1) m, d+(a+c-2) m, \ldots, d)$ and $\mu=\delta_{a+1}$. See Figure 15 .
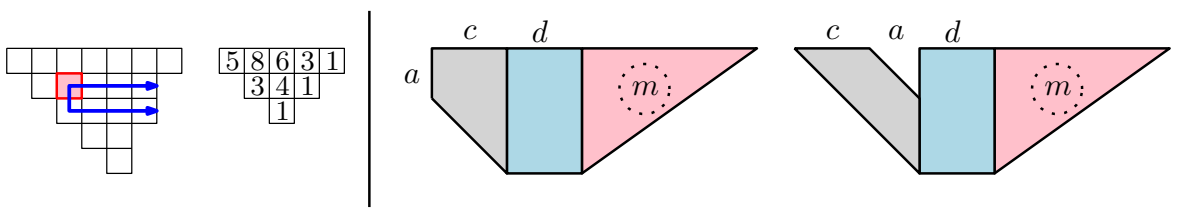

Figure 15. Left: example of the type $B$ hook of a cell $(i, j)$ of $\lambda$ of length 9 (cell $(3,3)$ is counted twice), and the type $B$ hook-lengths of the cells of the shifted shape $(5,3,1)$. Right: The shifted skew shape $\Lambda^{\nabla}(a, c, d, m)$ and the shape whose hooks appear in the product formula in Conjecture 9.6.

Computations using the Pfaffian formula for $g^{\lambda / \mu}$ (see [22, Thm. 7.5]), suggest the following conjectured ${ }^{(11)}$ product formula for these shifted skew shapes.

CONJECTURE 9.6. In the notation above, for $\pi=\Lambda^{\nabla}(a, c, d, m)$, we have:

$$
g^{\pi}=\frac{n !}{2^{a}} \cdot \frac{\Phi(2 a+c) \Phi(a)}{\Phi(2 a) \Phi(a+c)} \cdot \frac{\Xi(2 a) \beth(c)}{\beth(2 a+c)} \cdot \prod_{u \in \lambda \backslash\left(\delta_{a+c+1} / c^{a} \delta_{c+1}\right)} \frac{1}{h_{B}(u)} .
$$

See Figure 15 for an illustration of the cells of the shifted shape $\lambda$ whose hooklengths appear in the conjectured formula above. The special case $\pi=\Lambda^{\nabla}(a, c, d, 0)$ is the (conjugated) truncated rectangle shape, and was established by the third author using a different technique [61] and later in [25, Cor. 4.6] by yet again different methods. In particular, for $\pi=\Lambda^{\nabla}(a, c, 0,0)$, we obtain the product formula for shifted reverse hook in the example above. This both lends support to the conjecture and explains its appearance, which seemed out of place until now, see [1].

9.6. RACAH AND $q$-RACAH FORMULAS. In the Appendix of [10], the authors generalize the MacMahon box formula (3) to five variables, which they formulate in terms of lozenge tilings of $\mathrm{H}(a, b, c)$ with weights $F\left(p, q, u_{1}, u_{2}, u_{3}\right)$ given by products of certain elliptic functions (see Theorem 10.5 in [10]). Upon seeing our main technical tool, Theorem 3.10, Eric Rains noticed ${ }^{(12)}$ that there is a common special case of both formulas giving the $q$-Racah formula. In the notation of [10], let $u_{1}=u, u_{2}=u_{3}=0$, $p \rightarrow 0$ to get the following result:

\footnotetext{
${ }^{(11)}$ This conjecture was recently established in [29].

${ }^{(12)}$ Personal communication.
} 
Corollary 9.7 (Appendix to [10]). We have:

$$
\begin{aligned}
\sum_{\Pi \subset[a \times b \times c]} \prod_{(i, j, k) \in \Pi} \frac{q-q^{j+k-2 i} u}{1-q^{j+k-2 i+1} u} & \\
= & \prod_{(i, j, k) \in[a \times b \times c]} \frac{\left(1-q^{i+j+k-1}\right)\left(1-q^{j+k-i-1} u\right)}{\left(1-q^{i+j+k-2}\right)\left(1-q^{j+k-i} u\right)},
\end{aligned}
$$

where the summation is over all plane partitions $\Pi$ which fit inside the box $[a \times b \times c]$.

In the notation of Theorem 3.10, this identity follows by considering the hexagon $\mathrm{H}(b, c, a)$ ( $a$ is the height this time), so $\mu=b^{c}$, then setting $x_{i}=q^{-i+a}, y_{j}=u q^{j-a-1}$, and noting that the RHS factors. We omit the details.

For $u=0$, equation (53) gives the MacMahon $q$-formula (4), where the sum is over plane partitions, while the Racah formula follows by letting $q, u=q^{h} \rightarrow 1$

$$
\begin{aligned}
\sum_{\Pi \subset[a \times b \times c]} \prod_{(i, j, k) \in \Pi} \frac{j+k-2 i-1+h}{j+k-2 i+1+h} & \\
= & \prod_{(i, j, k) \in[a \times b \times c]} \frac{(i+j+k-1)(j+k-i-1+h)}{(i+j+k-2)(j+k-i+h)} .
\end{aligned}
$$

When $a=1$, this gives (16), since plane partitions of height 1 correspond to a single lattice path. Finally, when $h \rightarrow \infty$, this identity gives the MacMahon box formula (3).

9.7. Excited diagrams and Grothendieck Polynomials. In addition to Theorem 5.1, Knutson-Miller-Yong [31, Thm. 5.8] also gave a formula for the Grothendieck polynomials of vexillary permutations in terms of a larger class of diagrams called generalized excited diagrams. For the shape $\lambda / \mu$ these diagrams are defined as follows: for each active cell $(i, j)$ we do two types of generalized excited moves: (i) the usual move replacing $(i, j)$ by $(i+1, j+1)$, or (ii) the move which keeps $(i, j)$ and adds $(i+1, j+1)$ :

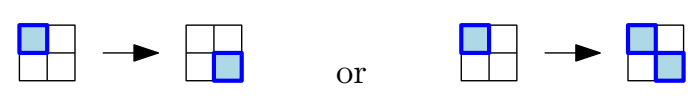

These diagrams were also studied by Kreiman [37] and they are in correspondence with set valued flagged tableaux. In [51], we use these diagrams to give a generalization of Naruse's formula (NHLF) and the analysis in Section 5 for Grothendieck polynomials.
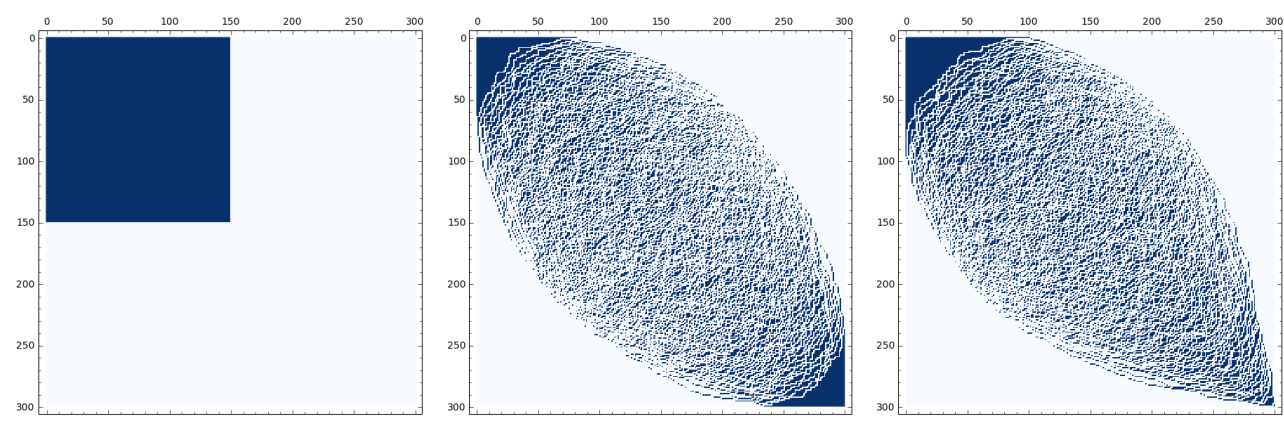

Figure 16. A random excited diagram of shape $300^{300} / 150^{150}$ (left), in the uniform (middle), and the hook-weighted distribution (right). 
9.8. Limit SHAPEs. Since excited diagrams are in bijection with lozenge tilings (see Theorem 7.2), one can translate known limit shape results for tilings into the language of excited diagrams. For example, the middle picture in Figure 16 is a random excited diagram of shape $300^{300} / 150^{150}$. These are obtained by running a Metropolis algorithm for $10^{10}$ steps. The visible limit shape is in fact a stretched circle.

Similarly, since $\operatorname{SYT}(\lambda / \mu)$ are enumerated by the weighted excited diagrams (by a product of hooks of the squares in the diagram), one can ask about limit shapes of hook weighted lozenge tilings. An example of a clearly visible limit shape is shown in the right picture in Figure 16. Both examples are a larger version of the lozenge tilings in Figure 2.
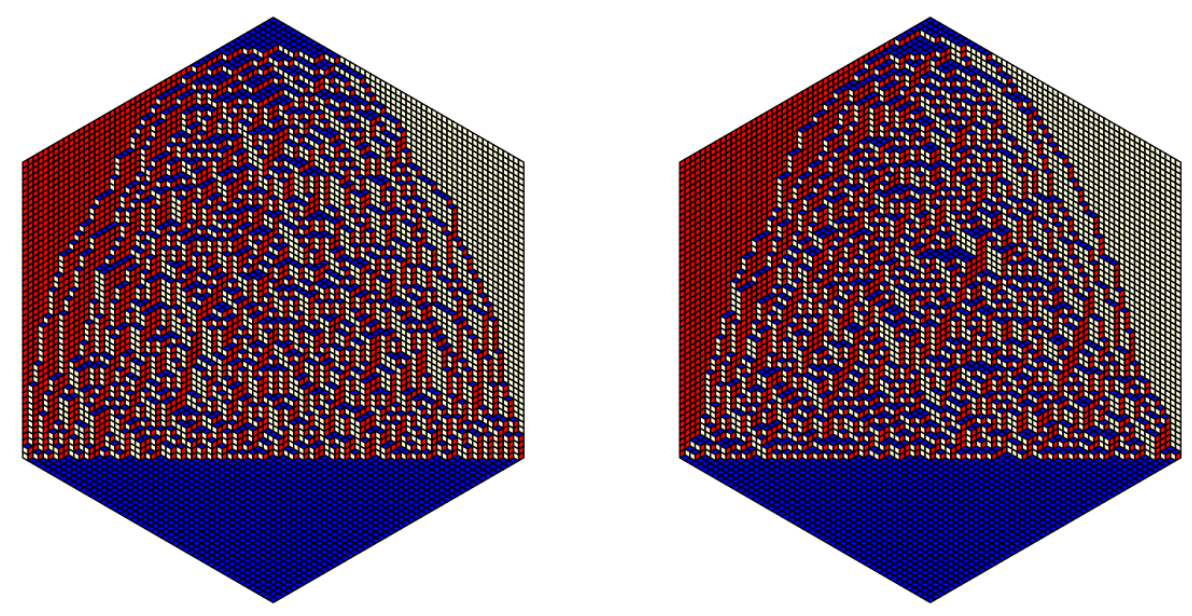

FiguRE 17. Uniform and hook-weighted lozenge tilings of the hexagon $\mathrm{H}(50,50,50)$ slanted by a diagonal.

Note that sometimes it is easier to analyze the limit shape for the lozenge tilings than for the other skew shapes like staircases. For examples, in Figure 17 we show two lozenge tilings of the hexagon $\mathrm{H}(50,50,50)$ slanted by a diagonal, one corresponding to the uniform excited diagram of the staircase $\pi=\delta_{150} / \delta_{50}$, and another with the hook weights. These random tilings are obtained by running a Metropolis algorithm for $3 \cdot 10^{9}$ steps. While the limit shapes have roughly similar outlines, in the uniform case the limit shape curves are visibly tangent to the vertical sides of the hexagon, and in the hook weighted case form an acute angle.

The observed behavior in the uniform case here is in line with the cases of lozenge tilings of polygonal regions; for such regions the frozen boundary is an inscribed algebraic curve as shown in e.g. [25, 26]. However, the hexagon with slanted diagonal is not a region which has been treated with any of the classical methods even in the uniform case. It would be interesting to obtain the exact form of the limit shape in the hook weighted case in connection with our detailed study of $f^{\pi}$ in $[53,55]$; see [64] for some related results.

Acknowledgements. We are grateful to Sami Assaf, Dan Betea, Sara Billey, Valentin Féray, Vadim Gorin, Zach Hamaker, Tri Lai, Leonid Petrov, Dan Romik, Luis Serrano, Richard Stanley, Hugh Thomas, Nathan Williams, Damir Yeliussizov and Alex Yong for useful comments and help with the references, and to Jane Austen [4] for the inspiration behind the first sentence. We are very thankful to Eric Rains for showing us the connections to Racah polynomials (see $§ 9.6$ ), to Christian Krattenthaler for 
pointing out to us the paper [36] and graciously allowing us to publish his conjectural formula (44), and to Jang Soo Kim for pointing us out that Corollary 1.1 appeared in [25]. We thank the anonymous referees for their careful reading, comments, and suggestions. The lozenge tilings in figures 2 and 17 were made using Sage and its algebraic combinatorics features developed by the Sage-Combinat community [68]. Martin Tassy generously helped us with the Metropolis simulations.

\section{REFERENCES}

[1] Ron Adin and Yuval Roichman, Standard Young tableaux, in Handbook of enumerative combinatorics, Discrete Math. Appl. (Boca Raton), CRC Press, Boca Raton, FL, 2015, pp. 895-974.

[2] Federico Ardila and Luis G. Serrano, Staircase skew Schur functions are Schur P-positive, J. Algebraic Combin. 36 (2012), no. 3, 409-423.

[3] Richard A. Askey and Ranjan Roy, Barnes G function, in NIST Handbook of Mathematical Functions, Cambridge University Press, 2010.

[4] Jane Austen, Pride and prejudice, vol. I.-III, T. Edgerton, Whitehall, London, England, 1813.

[5] Yuliy Baryshnikov and Dan Romik, Enumeration formulas for Young tableaux in a diagonal strip, Israel J. Math. 178 (2010), 157-186.

[6] Dan Betea, Elliptic combinatorics and Markov processes, Ph.D. thesis, California Institute of Technology (USA), 2012, p. 124.

[7] Sara C. Billey, Alexander E. Holroyd, and Benjamin J. Young, A bijective proof of Macdonald's reduced word formula, Algebr. Comb. 2 (2019), no. 2, 217-248.

[8] Sara C. Billey, William Jockusch, and Richard P. Stanley, Some combinatorial properties of Schubert polynomials, J. Algebraic Combin. 2 (1993), no. 4, 345-374.

[9] Alexei Borodin and Vadim Gorin, Shuffling algorithm for boxed plane partitions, Adv. Math. 220 (2009), no. 6, 1739-1770.

[10] Alexei Borodin, Vadim Gorin, and Eric M. Rains, q-distributions on boxed plane partitions, Sel. Math. (N.S.) 16 (2010), no. 4, 731-789.

[11] David M. Bressoud, Proofs and confirmations. The story of the alternating sign matrix conjecture, MAA Spectrum, Mathematical Association of America, Washington, DC; Cambridge University Press, Cambridge, 1999.

[12] Henry Cohn, Michael Larsen, and James Propp, The shape of a typical boxed plane partition, New York J. Math. 4 (1998), 137-165.

[13] Elizabeth Angela DeWitt, Identities relating Schur s-functions and Q-functions, Ph.D. thesis, University of Michigan (USA), 2012, p. 67.

[14] Jehanne Dousse and Valentin Féray, Asymptotics for skew standard Young tableaux via bounds for characters, https://arxiv.org/abs/1710.05652, to appear Proc. Amer. Math. Soc., 2017.

[15] Ilse Fischer, A bijective proof of the hook-length formula for shifted standard tableaux, https: //arxiv.org/abs/math/0112261, 2001.

[16] Philippe Flajolet and Robert Sedgewick, Analytic combinatorics, Cambridge University Press, Cambridge, 2009.

[17] Sergey Fomin and Anatol N. Kirillov, Reduced words and plane partitions, J. Algebraic Combin. 6 (1997), no. 4, 311-319.

[18] Vadim Gorin and Greta Panova, Asymptotics of symmetric polynomials with applications to statistical mechanics and representation theory, Ann. Probab. 43 (2015), no. 6, 3052-3132.

[19] W. Timothy Gowers, The two cultures of mathematics, in Mathematics: frontiers and perspectives, Amer. Math. Soc., Providence, RI, 2000, pp. 65-78.

[20] Curtis Greene, Albert Nijenhuis, and Herbert S Wilf, A probabilistic proof of a formula for the number of Young tableaux of a given shape, Adv. Math. 31 (1979), no. 1, 104-109.

[21] Takeshi Ikeda and Hiroshi Naruse, Excited Young diagrams and equivariant Schubert calculus, Trans. Amer. Math. Soc. 361 (2009), no. 10, 5193-5221.

[22] Vladimir Nikolaevich Ivanov, Interpolation analogues of Schur Q-functions, Zap. Nauchn. Sem. S.-Peterburg. Otdel. Mat. Inst. Steklov. (POMI) 307 (2004), no. 10, 99-119, 281-282.

[23] Noriaki Kawanaka, A q-series identity involving Schur functions and related topics, Osaka J. Math. 36 (1999), no. 1, 157-176.

[24] Richard Kenyon, Lectures on dimers, in Statistical mechanics, IAS/Park City Math. Ser., vol. 16, Amer. Math. Soc., Providence, RI, 2009, pp. 191-230.

[25] Richard Kenyon and Andrei Okounkov, Limit shapes and the complex Burgers equation, Acta Math. 199 (2007), no. 2, 263-302. 
[26] Richard Kenyon, Andrei Okounkov, and Scott Sheffield, Dimers and amoebae, Ann. of Math. (2) 163 (2006), no. 3, 1019-1056.

[27] Jang Soo Kim and Suho Oh, The Selberg integral and Young books, J. Combin. Theory Ser. A 145 (2017), 1-24.

[28] Jang Soo Kim and Soichi Okada, A new q-Selberg integral, Schur functions, and Young books, Ramanujan J. 42 (2017), no. 1, 43-57.

[29] Jang Soo Kim and Meesue Yoo, Product formulas for certain skew tableaux, https://arxiv. org/abs/1806.01525, 2018.

[30] Sergey Kitaev, Patterns in permutations and words, Monographs in Theoretical Computer Science. An EATCS Series, Springer, Heidelberg, 2011, With a foreword by Jeffrey B. Remmel.

[31] Allen Knutson, Ezra Miller, and Alexander Yong, Gröbner geometry of vertex decompositions and of flagged tableaux, J. Reine Angew. Math. 630 (2009), 1-31.

[32] Allen Knutson and Terence Tao, Puzzles and (equivariant) cohomology of Grassmannians, Duke Math. J. 119 (2003), no. 2, 221-260.

[33] Christian Krattenthaler, Generating functions for plane partitions of a given shape, Manuscripta Math. 69 (1990), no. 2, 173-201.

[34] _ Advanced determinant calculus, Sém. Lothar. Combin. 42 (1999), B42q (67 pages).

[35] - Advanced determinant calculus: a complement, Linear Algebra Appl. 411 (2005), 68166.

[36] Christian Krattenthaler and Michael J. Schlosser, The major index generating function of standard Young tableaux of shapes of the form "staircase minus rectangle", in Ramanujan 125, Contemp. Math., vol. 627, Amer. Math. Soc., Providence, RI, 2014, pp. 111-122.

[37] Victor Kreiman, Schubert classes in the equivariant K-theory and equivariant cohomology of the Grassmannian, https://arxiv.org/abs/math/0512204, 2005.

[38] Greg Kuperberg, Another proof of the alternating-sign matrix conjecture, Internat. Math. Res. Notices (1996), no. 3, 139-150.

[39] _ Symmetry classes of alternating-sign matrices under one roof, Ann. of Math. (2) 156 (2002), no. 3, 835-866.

[40] Tri Lai, Enumeration of tilings of quasi-hexagons, hexagonal dungeons, quartered hexagons, and variants of the Aztec diamond, Ph.D. thesis, Indiana University (USA), 2014.

[41] _ A q-enumeration of lozenge tilings of a hexagon with three dents, Adv. in Appl. Math. 82 (2017), 23-57.

[42] Venkatramani Lakshmibai, Komaranapuram N. Raghavan, and Parameswaran Sankaran, Equivariant Giambelli and determinantal restriction formulas for the Grassmannian, Pure Appl. Math. Q. 2 (2006), no. 3, 699-717, Special Issue: In honor of Robert D. MacPherson. Part 1.

[43] Alain Lascoux, Polynomials, monograph draft, http://www-igm.univ-mlv.fr/ al/ARTICLES/ CoursYGKM.pdf, 2013.

[44] Alain Lascoux and Marcel-Paul Schützenberger, Polynômes de Schubert, C. R. Acad. Sci. Paris Sér. I Math. 294 (1982), no. 13, 447-450.

[45] - Structure de Hopf de l'anneau de cohomologie et de l'anneau de Grothendieck d'une variété de drapeaux, C. R. Acad. Sci. Paris Sér. I Math. 295 (1982), no. 11, 629-633.

[46] Ian Grant Macdonald, Notes on Schubert polynomials, LaCIM, UQAM, 1991.

[47] Laurent Manivel, Symmetric functions, Schubert polynomials and degeneracy loci, SMF/AMS Texts and Monographs, vol. 6, American Mathematical Society, Providence, RI; Société Mathématique de France, Paris, 2001, Translated from the 1998 French original by John R. Swallow, Cours Spécialisés [Specialized Courses], 3.

[48] Grigory Merzon and Evgeny Smirnov, Determinantal identities for flagged Schur and Schubert polynomials, Eur. J. Math. 2 (2016), no. 1, 227-245.

[49] Alexander I. Molev and Bruce E. Sagan, A Littlewood-Richardson rule for factorial Schur functions, Trans. Amer. Math. Soc. 351 (1999), no. 11, 4429-4443.

[50] Alejandro H. Morales, Zach Hamaker, Igor Pak, and Luis G. Serrano, in preparation.

[51] Alejandro H. Morales, Igor Pak, and Greta Panova, Hook formulas for skew shapes IV. Increasing tableaux and factorial Grothendieck polynomials, in preparation.

[52] - Hook formulas for skew shapes II. Combinatorial proofs and enumerative applications, SIAM J. Discrete Math. 31 (2017), no. 3, 1953-1989.

[53] - Asymptotics of the number of standard Young tableaux of skew shape, European J. Combin. 70 (2018), 26-49.

[54] _ Hook formulas for skew shapes I. q-analogues and bijections, J. Combin. Theory Ser. A 154 (2018), 350-405.

[55] _ Asymptotics of principal evaluations of Schubert polynomials for layered permutations, Proc. Amer. Math. Soc. 147 (2019), no. 4, 1377-1389. 
[56] Alejandro H. Morales, Igor Pak, and Martin Tassy, Asymptotics for the number of standard tableaux of skew shape and for weighted lozenge tilings, https://arxiv.org/abs/1805.00992, 2018.

[57] Hiroshi Naruse, Schubert calculus and hook formula, 2014, talk at 73rd Sém. Lothar. Combin., Strobl, Austria; slides available at https://www.emis.de/journals/SLC/wpapers/s73vortrag/ naruse.pdf.

[58] Hiroshi Naruse and Soichi Okada, Skew hook formula for d-complete posets, 2018, https:// arxiv.org/abs/1802.09748.

[59] Jean-Christophe Novelli, Igor Pak, and Alexander V. Stoyanovskii, A direct bijective proof of the hook-length formula, Discrete Math. Theor. Comput. Sci. 1 (1997), no. 1, 53-67.

[60] Igor Pak, Tile invariants: new horizons, Theoret. Comput. Sci. 303 (2003), no. 2-3, 303-331, Tilings of the plane.

[61] Greta Panova, Tableaux and plane partitions of truncated shapes, Adv. Appl. Math. 49 (2012), no. 3-5, 196-217.

[62] Leonid Petrov, Asymptotics of random lozenge tilings via Gelfand-Tsetlin schemes, Probab. Theory Relat. Fields 160 (2014), no. 3-4, 429-487.

[63] Robert A. Proctor, New symmetric plane partition identities from invariant theory work of De Concini and Procesi, European J. Combin. 11 (1990), no. 3, 289-300.

[64] Dan Romik, Arctic circles, domino tilings and square Young tableaux, Ann. Probab. 40 (2012), no. $2,611-647$.

[65] Gian-Carlo Rota, Ten lessons I wish I had been taught, in Indiscrete thoughts, Birkhäuser, 1997, pp. 195-203.

[66] Bruce Sagan, On selecting a random shifted Young tableau, J. Algorithms 1 (1980), no. 3 213-234.

[67] _ The symmetric group, second ed., Graduate Texts in Mathematics, vol. 203, SpringerVerlag, New York, 2001, Representations, combinatorial algorithms, and symmetric functions.

[68] Sage-Combinat community, Sage-Combinat: enhancing Sage as a toolbox for computer exploration in algebraic combinatorics, 2017, https://wiki.sagemath.org/combinat.

[69] Luis G. Serrano and Christian Stump, Maximal fillings of moon polyominoes, simplicial complexes, and Schubert polynomials, Electron. J. Combin. 19 (2012), no. 1, P16 (18 pages).

[70] Neil James Alexander Sloane, The Online Encyclopedia of Integer Sequences, https://oeis. org/.

[71] Richard P. Stanley, Plane partitions: past, present, and future, in Combinatorial Mathematics: Proceedings of the Third International Conference (New York, 1985), Ann. New York Acad. Sci., vol. 555, New York Acad. Sci., New York, 1989, pp. 397-401.

[72] _ Enumerative combinatorics, vol. 2, Cambridge University Press, 1999.

[73] _ A survey of alternating permutations, in Combinatorics and graphs, Contemp. Math., vol. 531, Amer. Math. Soc., Providence, RI, 2010, pp. 165-196.

[74] _ Enumerative combinatorics, second ed., vol. 1, Cambridge University Press, 2012.

[75] _ Some Schubert shenanigans, https://arxiv.org/abs/1704.00851, 2017.

[76] John R. Stembridge, Nonintersecting paths, pfaffians, and plane partitions, Adv. Math. 83 (1990), no. 1, 96-131.

[77] William P. Thurston, Groups, tilings and finite state automata, Geometry Computing Group, 1989.

[78] Michelle L. Wachs, Flagged Schur functions, Schubert polynomials, and symmetrizing operators, J. Combin. Theory Ser. A 40 (1985), no. 2, 276-289.

[79] Anna E. Weigandt, Schubert polynomials, 132-patterns, and Stanley's conjecture, Algebr. Comb. 1 (2018), no. 4, 415-423.

[80] Alexander Woo, Catalan numbers and Schubert polynomials for $w=1(n+1) \ldots 2$, https: //arxiv.org/abs/math/0407160, 2004. 
Alejandro H. Morales, University of Massachusetts, Amherst, Department of Mathematics and Statistics, Amherst, MA, 01002, USA

E-mail : ahmorales@math.umass.edu

IGOR PAK, University of California, Los Angeles, Department of Mathematics, Los Angeles, CA, 90095, USA

E-mail : pak@math.ucla.edu

Greta Panova, University of Southern California, Department of Mathematics, Los Angeles, CA, 90089, USA

E-mail : gpanova@usc.edu 